\title{
Knowledge transmission patterns at the border: ethnobotany of Hutsuls living in the Carpathian Mountains of Bukovina (SW Ukraine and NE Romania)
}

\author{
Giulia Mattalia ${ }^{1,2^{*}}$, Nataliya Stryamets ${ }^{1}$, Andrea Pieroni ${ }^{3,4+}$ and Renata Sõukand ${ }^{1+}$
}

\begin{abstract}
Background: Cross-border research is a novel and important tool for detecting variability of ecological knowledge. This is especially evident in regions recently divided and annexed to different political regimes. Therefore, we conducted a study among Hutsuls, a cultural and linguistic minority group living in Northern and Southern Bukovina (Ukraine and Romania, respectively). Indeed, in the 1940s, a border was created: Northern Bukovina was annexed by the USSR while Southern Bukovina remained part of the Kingdom of Romania. In this research, we aim to document uses of plants for food and medicinal preparations, discussing the different dynamics of Local Ecological Knowledge (LEK) transmission among Hutsuls living in Ukraine and Romania.

Methods: Field research was conducted using convenience and snowball sampling techniques to recruit 31 Hutsuls in Ukraine and 30 in Romania for participation in semi-structured interviews regarding the use of plants for medicinal and food preparation purposes and the sources of such knowledge.

Results: The interviews revealed that, despite a common cultural and linguistic background, ethnobotanical knowledge transmission occurs in different ways on each side of the border. Family is a primary source of ethnobotanical knowledge transmission on both sides of the border; however, in Romania, knowledge from other sources is very limited, whereas in Ukraine interviewees reported several other sources including books, magazines, newspapers, the Internet and television. This is especially evident when analysing the wild plants used for medicinal purposes as we found 53 taxa that were common to both, 47 used only in Ukraine and 11 used only in Romania. While Romanian Hutsuls used almost exclusively locally available plants, Ukrainian Hutsuls often reported novel plants such as Aloe vera, Aronia melanocarpa and Elaeagnus rhamnoides. Knowledge related to these plants was transferred by sources of knowledge other than oral transmission among members of the same family. Therefore, this may imply hybridization of the local body of knowledge with foreign elements originating in the Soviet context which has enriched the corpus of ethnobotanical knowledge held by Ukrainian Hutsuls.

(Continued on next page)
\end{abstract}

\footnotetext{
* Correspondence: giulia.mattalia@unive.it

${ }^{\dagger}$ Andrea Pieroni and Renata Soukand contributed equally to this work.

'Department of Environmental Sciences, Informatics and Statistics, Ca' Foscari University of Venice, Via Torino 155, 30172, Mestre, Venezia, Italy

${ }^{2}$ Autonomous University of Barcelona, Institute of Environmental Science and Technology, 08193, Bellaterra, Cerdanyola del Vallès, Spain

Full list of author information is available at the end of the article
}

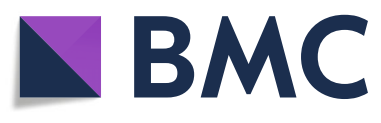

(c) The Author(s). 2020 Open Access This article is licensed under a Creative Commons Attribution 4.0 International License, which permits use, sharing, adaptation, distribution and reproduction in any medium or format, as long as you give appropriate credit to the original author(s) and the source, provide a link to the Creative Commons licence, and indicate if changes were made. The images or other third party material in this article are included in the article's Creative Commons licence, unless indicated otherwise in a credit line to the material. If material is not included in the article's Creative Commons licence and your intended use is not permitted by statutory regulation or exceeds the permitted use, you will need to obtain permission directly from the copyright holder. To view a copy of this licence, visit http://creativecommons.org/licenses/by/4.0/ The Creative Commons Public Domain Dedication waiver (http://creativecommons.org/publicdomain/zero/1.0/) applies to the data made available in this article, unless otherwise stated in a credit line to the data. 


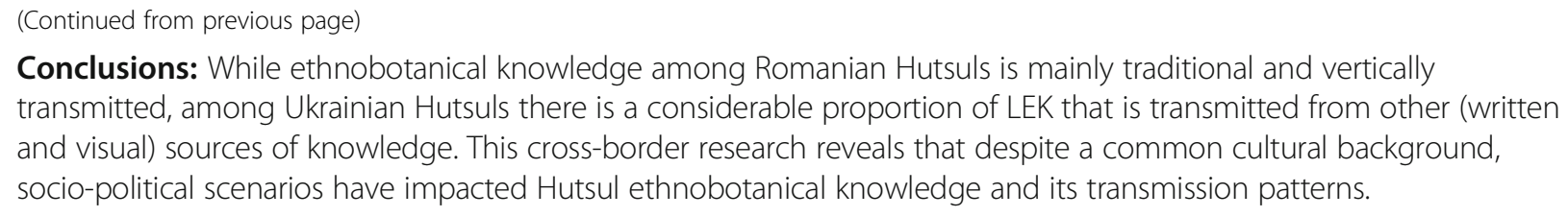

Keywords: Biocultural diversity, Ecological Knowledge, LEK, Minority groups, TEK, Wild food plants, Wild medicinal plants

\section{Background}

The current global changes demand thorough analysis of not only ecological knowledge per se but also how such knowledge is produced, shared and used [1]. Indeed, ecological knowledge is a valuable system, which can significantly contribute to a better understanding of the current socio-economic and environmental changes occurring all over the word [2,3]. These bodies of knowledge are seriously endangered by urbanization and the increasing adoption of new modes of life disconnected from local ecosystem dynamics and resources [4]. In addition, a widespread tendency of formal education (e.g. literature) to downplay local resources and knowledge has been observed [5], thus leading to knowledge homogenization and standardization $[6,7]$.

Ethnobotanical knowledge can be considered as part of local ecological knowledge (LEK) and it can be, but not necessarily is, regarded as traditional. Indeed, LEK 'concerns the interplay among organisms and between organisms and their environment. LEK may be a mix of scientific and practical knowledge; it is site-specific and often involves a belief component' [8]. Berkes [9] defined traditional ecological knowledge (TEK) as 'a cumulative body of knowledge and beliefs, handed down through generations by cultural transmission, about the relationship of living beings (including humans) with one another and with their environment.' As observed by Olsson and Folke [8], the specific characteristics of TEK lie in its 'historical and cultural continuity of resource use'.

These two definitions of ecological knowledge are not in opposition, but rather, as largely accepted by the majority of ethnobiology scholars, both TEK and LEK define a complex and heterogeneous body of folk knowledge, practices and beliefs related to the natural environment. However, solely for the instructive aim of a better understanding of the different 'nature' of these bodies of knowledge in the current context, in this article we adopt the term TEK when referring to a system in which knowledge and practices are mainly orally transmitted (e.g. pre-industrial contexts), while we use the term LEK to refer to a system in which the borders between written (or in other words 'standardized') and oral forms of knowing nature and practicing this knowledge are more blurred.
Van den Boog et al. ([10] and references within) discussed and categorized the dynamics of LEK transmission into vertical (between generations within the family), horizontal (between people of the same generation) and oblique (between generations not belonging to the same family). The evolving dynamics of ethnobotanical knowledge transmission have been found to be affected by not only exposure to a market economy [11, 12] but also socio-economic changes [13] and political circumstances [7].

Hutsuls are an ethnic group living in the Carpathian Mountains of Ukraine and to a lesser extent Romania. These communities have been recently studied from an ethnographic perspective $[14,15]$ as well as an ethnobotanical one $[6,16]$.

Over the last few years, cross-border ethnobotany has received increasing attention from scholars [16-18] as it is an excellent tool for exploring the effects of different social and political contexts on LEK. In this study, we examine culturally homogenous Hutsul communities living in similar mountain landscapes (Fig. 1), yet separated by a border created in the 1940s when Northern Bukovina was annexed by the Soviet Union and Southern Bukovina remained part of the Kingdom of Romania. The aim of this study, therefore, is to document and discuss the different dynamics of LEK transmission among Hutsuls living in Ukraine and Romania and to explore whether the different social, political and economic conditions that developed after border creation have affected these dynamics.

\section{Methods}

\section{Study area and historical background}

The interviews were conducted in Bukovina (Fig. 2), a region of Eastern Europe characterized by an extensive forested area especially in proximity to the Carpathian Mountains. This region belonged to the Hapsburg Empire for over 140 years until 1918, when it became part of the new Kingdom of Romania. In 1940, the Ribbentrop Molotov Pact split this region into two parts: Northern Bukovina was annexed by the USSR and thus a new border was created. After a few years of uncertain borders, in 1944 Southern Bukovina was assigned to Romania, and since 2007 it 


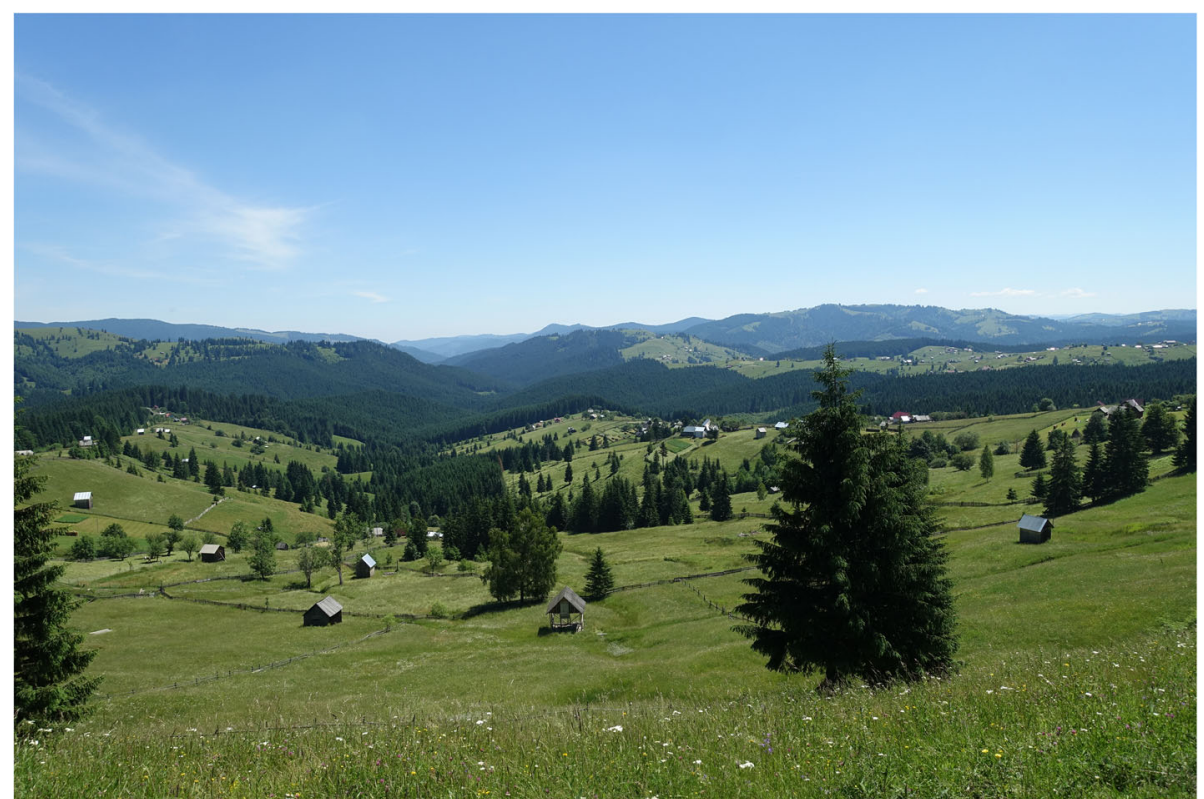

Fig. 1 Bukovinian Carpathian landscape, Lupcina, Romania; July 2019; Photo by N. Stryamets

has been a member of the European Union, whereas Northern Bukovina, after the collapse of the Soviet Union in 1991, became part of independent Ukraine. While Northern Bukovina underwent a process of homogenization and centralization promoted by the USSR, Southern Bukovina was not heavily affected by Romanian collectivization policies due to its limited economic interest.

Over one century ago, Bukovina was a multicultural and multi-religious mosaic composed of Romanians, Ukrainians, Jews, Armenians, Roma people, Hungarian Székelys, Russian Old Believers (Lipovans), Germans (mainly clerks), Slovaks, Poles and Tatars [19, 20].

Currently, only a small portion of such cultural diversity is maintained, as linguistic and ethnic minorities have undergone a process of homogenization [21]. Among these minorities are Hutsuls who live in the Carpathian Mountains of the Suceava district of Romania and the Cernivtci, Ivano-Frankivs'k and Zakarpatska provinces of Ukraine. Hutsuls speak a local language which they themselves consider to include elements of Ukrainian, Polish, German and Hungarian [14]. In Romania, children are taught both in Romanian and Ukrainian in school, while at home they mainly speak the Hutsul language. In Ukraine, they attend school in Ukrainian and use Hutsul for informal conversations. The main economic activities of both Romanian and Ukrainian Hutsuls are small-scale mixed farming and non-wood forest product exploitation. All interviewed Hutsuls belonged to the Orthodox Church.

The climate of the area is classified as Dfb, a humid continental climate, without a dry season and with warm summers. Annual precipitation is around $775 \mathrm{~mm}$, which is mainly concentrated in June and July. The coldest month is January when average temperature is -5.5 ${ }^{\circ} \mathrm{C}$ and the warmest is July at $16{ }^{\circ} \mathrm{C}$.

\section{Sampling and interviews}

Sixty-one Hutsul informants were interviewed in Romanian and Ukrainian Bukovina between June 2018 and July 2019. Thirty in-depth interviews where gathered in the municipalities of Brodina, Ulma and Izvoarele Sucevei, in the district of Suceava, Northern Romania, while 31 interviews were conducted in the districts of Putyla (main villages in which interviews were conducted include Kyselytsi, Shepit, Serhii, Foshky, Parkulyna, Ryzha) and Vyzhnytsia (Dolishnii Shepit) in the province of Cernivtci, Southern Ukraine (Fig. 3). Altitude of the villages ranges from 600 to $1000 \mathrm{~m}$ a.s.l.

Informants were conveniently selected (we interviewed people walking on the street, working in their gardens or talking in cafes) and when possible we used the snowball method. We strictly followed the ethical guidelines prescribed by the International Society of Ethnobiology (ISE 2006) and the study protocol was approved by the Ethical Committee of Ca' Foscari University of Venice.

We used semi-structured interviews to obtain qualitative and quantitative data regarding the use of plants for culinary and medicinal purposes (starting with culinary use). Specifically, we asked interviewees what plants they harvest, for what purpose and how they prepare them. In addition, we asked informants the source of such knowledge, i.e. from whom or where did they learn it. In some cases, we deemed it useful to draw a timeline 

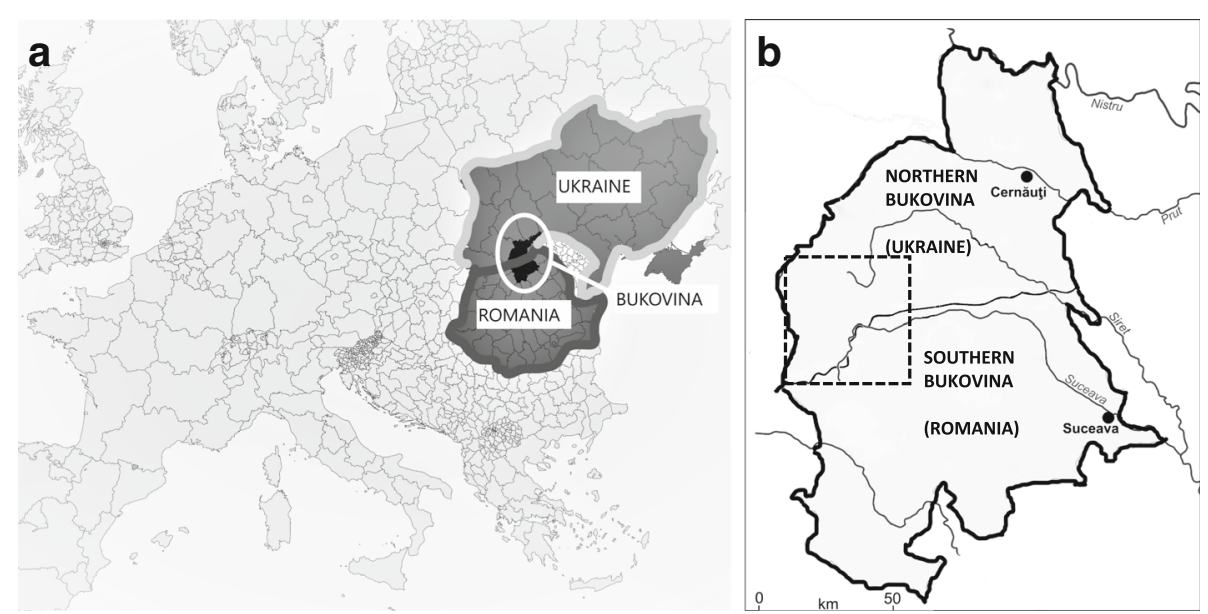

Fig. 2 a, b Maps of the study area

indicating when each informant started using each plant. When possible, we harvested the mentioned plants together with the interviewees in order to collect and identify voucher specimens. Voucher specimens collected in Ukraine are stored in the 'Roztochya' Nature Reserve (Ukraine) bearing codes NB001-NB259, while those collected in Romania are stored in the Herbarium of Ca' Foscari University of Venice (Italy) bearing codes SB001-SB094. Voucher specimens were identified using the 'The Plant List' [22] and 'Flora Europaea' [23]. Plant families were classified according to Stevens [24]. Interviews were held in Romanian or Ukrainian according to the preference of the interviewees. In Romania, many interviewees answered using a mixture of Romanian, Ukrainian or the Hutsul language, while in Ukraine they tended to stick to Ukrainian.

\section{Data analysis}

Gathered data on the use of plants for various purposes were entered into an Excel spreadsheet. We structured emic categories into detailed use-reports (DUR), where each interviewee mentioned a specific use (e.g. abdominal pain) of a plant part (e.g. aerial parts or roots) for a specific preparation (e.g. tea or infused in alcohol). The spreadsheet

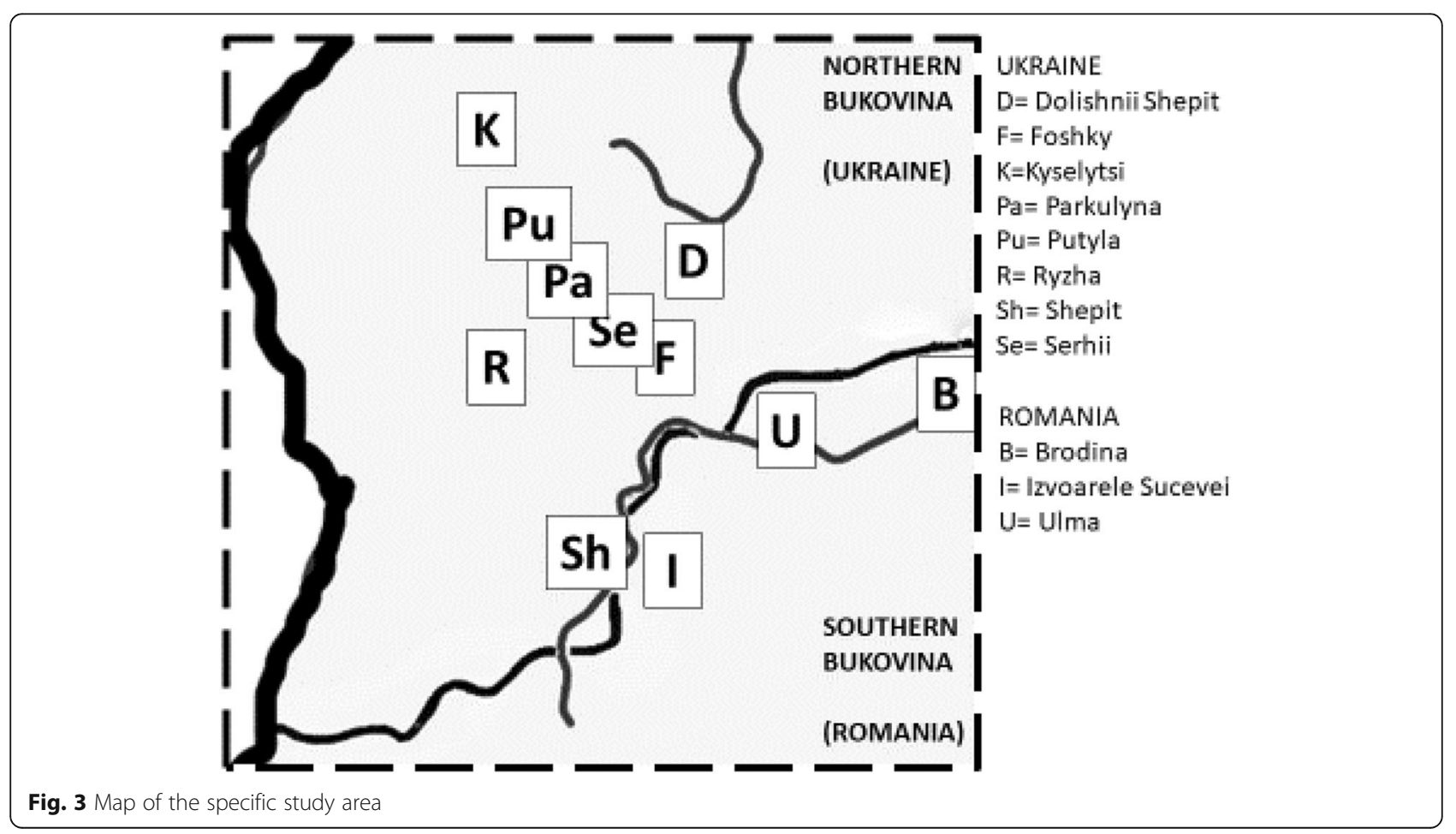


included informant code, language of the interview, plant parts used, scientific name, family assignment, local name (Ukrainian and Hutsul names were transliterated using the system adopted by the Cabinet of Ministers of Ukraine [25]), mode of preparation, time of use (always, in the past, recently abandoned, recently adopted), medicinal use, food use, source of knowledge and comments. In addition, for medicinal uses, we related ICD-11 medicinal categories [26] to reported emic categories (e.g. good for the stomach) for better comparison. In addition to the ICD-11 (International Classification of Diseases) categories, we included a general health category including mainly 'general symptoms' and some unspecified emic categories.

We considered only wild plants for food purposes, while we also included cultivated plants for medicinal purposes. We considered as 'wild' plants that grow without intended cultivation. This category mainly consists of native and naturalized species, but also plants not cultivated for food or medicinal purposes (e.g. Tilia cordata), as well as species that are generally gathered from the wild but can also be cultivated (e.g. Rubus idaeus).

To compare Romanian and Ukrainian Hutsuls, we calculated the Jaccard Similarity Index (JI) following the methodology of González-Tejero et al. [27]:

$$
\mathrm{JI}=(C /(A+B-C)) \times 100
$$

where $A$ is the number of species in sample $\mathrm{A}, B$ is the number of species in sample $B$ and $C$ is the number of species common to both $\mathrm{A}$ and $\mathrm{B}$. An index value close to 100 indicates that the two groups are very similar, while a value close to 0 indicates that are very different.

In order to calculate the proportion of each knowledge transmission strategy, we assigned a total of 1 point to each source of knowledge indicated by the interviewee. Therefore, if the interviewee reported one source (e.g. parents), we assigned a value of 1 ; two sources (e.g. books and grandparents), we assigned 0.5 to each; three sources, 0.33 to each; and four sources, 0.25 to each. Later, we summed these values in the emic categories of knowledge source mentioned by the interviewees on both sides of the border.

\section{Results and discussion}

We recorded a total of 118 food and medicinal plants from 107 genera and 53 families. The most well represented families were Asteraceae, Rosaceae and Lamiaceae. Among Hutsuls of Northern Bukovina, we recorded 107 taxa, while there were 72 taxa among Hutsuls of Southern Bukovina, and 60 taxa common to both. The most used plants were Vaccinium myrtillus, Rubus idaeus and Urtica dioica. These were the most used in both Northern and Southern Bukovina and thus we can confirm their importance as Hutsul cultural markers as previously suggested by Sõukand and Pieroni [16].

\section{Food taxa}

The interviewed Hutsuls reported a total of 47 taxa used for food preparations (Table 1). Twenty-six taxa were found on both sides of the border, eight taxa were reported only in Romania and 13 only in Ukraine (Fig. 4). When considering only the plants mentioned by at least $10 \%$ of the interviewees (three), Romanian Hutsuls mentioned ten taxa, Ukrainians five taxa and 16 were common to both. The Jaccard Similarity Index (JI) for the two groups was 55 when based on all the taxa, while an index value of 51 was observed when considering only the taxa mentioned by $10 \%$ of interviewees. The most common taxa correspond to those most used overall (Vaccinium myrtillus, Rubus idaeus and Urtica dioica), although among Romanian Hutsuls, Fagus sylvatica was also very common as its wood was used for smoking pork meat, which is one of the most traditional Hutsul preparations, as well as to flavour soups. Rumex acetosa was very often reported by Ukrainian Hutsuls (but never by Romanian Hutsuls) as an ingredient for soups. The most common food purpose was recreational tea, a preparation used for 30 taxa. Tea was widely reported in Northern Bukovina where 23 taxa were mentioned, of which 13 were shared with Southern Bukovina, for a total of 81 DUR. In addition, six taxa were reported only among Hutsuls in Southern Bukovina for a total of 19 taxa and 65 DUR. In line with Sõukand et al. [28], the main represented families for recreational teas were Rosaceae followed by Asteraceae and Lamiaceae. Another common preparation was jam which predominated in Romania (82 DUR) and included eight taxa, five of which were common to both communities (Fragaria vesca; Rubus caesius; Rubus idaeus; Vaccinium myrtillus; Vaccinium vitis-idaea). Among the taxa used for jams exclusively prepared by Romanian Hutsuls, we found the young sprouts of Picea abies, which are harvested in June and can also be used for making medicinal syrup, and the petals of Rosa rugosa and Rosa centifolia, which are used for jams and teas almost exclusively by Romanian Hutsuls. In addition, the flowers of Taraxacum officinale were also used for the preparation of jam in Southern Bukovina. Another common use of wild food plants was seasoning, and in particular Thymus spp. and Armoracia rusticana which were used in both communities. Actually, Armoracia rusticana was reported by Ukrainian Hutsuls for 'квашення' (kvashennya), which is a lacto-fermented preparation of cucumbers, tomatoes, cabbage or other vegetables, a typical recipe common in Romania under the name of 'muraturi'. For this preparation, Hutsuls from both communities reported the use of Armoracia rusticana roots (in Ukraine leaves were 
Table 1 Recorded food taxa in Northern and Southern Bukovina. DUR Detailed Use Reports; RO Romanian Hutsuls; UA Ukrainian Hutsuls

\begin{tabular}{l} 
Latin name, Family and vouc \\
\hline Acer spp. including Acer \\
pseudoplatanus L. \\
(Sapindaceae) \\
NB225; NB226 \\
Achillea millefolium L. \\
(Asteraceae) \\
SB011; SB050; SB074 \\
NB007; NB017; NB039 \\
Armoracia rusticana P.Gaertn. \\
B.Mey. \& Scherb. \\
(Brassicaceae) \\
SB031 \\
NB028
\end{tabular}

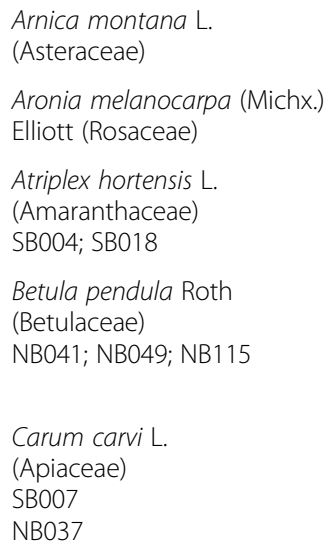

Equisetum spp. including Equisetum arvense L.; Equisetum

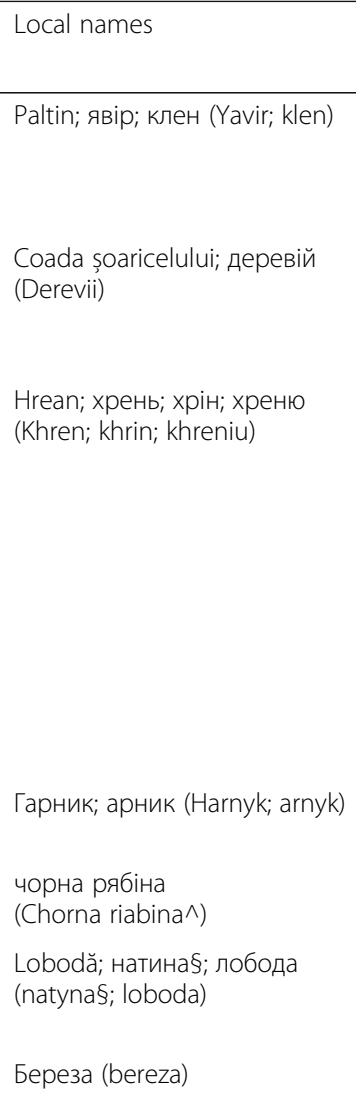

(2)

Гарник; арник (Harnyk; arnyk)
чopна рябіна
(Chorna riabina^)
Lobodă; натина§; лобода
(natyna§; loboda)
Береза (bereza)

Береза (bereza)

Săcărică; Cmin; Hmel; хміль§; тмин; хміль польовийङ; кмин

(Khmil§; tmyn; Khmil polovyi§; kmyn)

Used part(s) Preparation

Sap Drink

Aerial parts Tea

(dried)

Roots

Salad (with
beetroots)

Seasoning

Raw

DUR

$\overline{R O U A}$

4

Pickles (cucumbers, tomatoes)

Fermenting

Leaves

Pickles (cucumbers) 1

Whole plant

Seasoning

Aerial parts

Tea

3

(dried)

Fruits

Kvas

Aerial parts

Soup

Leaves

Sarmale

8

Sap

Drink

2

Strong alcohol

Leaves

Mixed tea

Aerial parts

Tea

Seasoning

3

Seeds

Lobodă; натина§; лебеда (Natyna§; $\quad$ Aerial parts lebeda)

Tea

2

Seasoning

2

Fermenting

3

Pickles

Bread additive

Soup

Stewed (with cream) 1

Seasoning (dried) 1

петрів батіг (Petriv batih)

Aerial parts

Tea

колєндра (Koliendra)

Seeds

Smoking (meat

Alune

Fruits

Raw

5

Глід (Hlid)

Fruits

Tea

іван чай, демник§; ;имник§ (ivan chai, demnyk§; dymnyk§)

Aerial parts

Tea

Barba ursului; Coada calului; 
Table 1 Recorded food taxa in Northern and Southern Bukovina. DUR Detailed Use Reports; RO Romanian Hutsuls; UA Ukrainian Hutsuls (Continued)

\begin{tabular}{|c|c|c|c|c|c|}
\hline \multirow[t]{2}{*}{ Latin name, Family and voucher specimens } & \multirow[t]{2}{*}{ Local names } & \multirow[t]{2}{*}{ Used part(s) } & \multirow[t]{2}{*}{ Preparation } & \multicolumn{2}{|c|}{ DUR } \\
\hline & & & & $\overline{\mathrm{RO}}$ & $\overline{U A}$ \\
\hline $\begin{array}{l}\text { sylvaticum L } \\
\text { (Equisetaceae) } \\
\text { SB020 } \\
\text { NB068, NB093, NB113, NB114 }\end{array}$ & $\begin{array}{l}\text { Padivolos; хвощ польловий, } \\
\text { падиволос§; } \\
\text { (Khvoshch pollovyi, padyvolos) }\end{array}$ & & & & \\
\hline $\begin{array}{l}\text { Fagus sylvatica L. } \\
\text { (Fagaceae) } \\
\text { SB060, } \\
\text { NB168 }\end{array}$ & Fag; бук (Buk) & Wood & Smoking (meat) & 19 & 1 \\
\hline \multirow{8}{*}{$\begin{array}{l}\text { Fragaria vesca L. } \\
\text { (Rosaceae) } \\
\text { SB094 } \\
\text { NB004, NB015, NB056 }\end{array}$} & \multirow{8}{*}{ 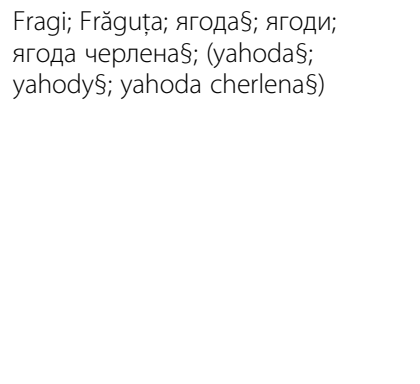 } & \multirow[t]{8}{*}{ Fruits } & Raw & 4 & 2 \\
\hline & & & Jam & 14 & 9 \\
\hline & & & Compote & 3 & 1 \\
\hline & & & Dessert & 2 & \\
\hline & & & Tea & 2 & \\
\hline & & & Juice & 1 & 1 \\
\hline & & & Syrup & 2 & \\
\hline & & & Frozen & & 1 \\
\hline $\begin{array}{l}\text { Gentiana spp. possibly including Gentiana lutea L. and } \\
\text { Gentiana asclepiadea L. } \\
\text { (Gentianaceae) }\end{array}$ & Gingiura & Aerial parts & $\begin{array}{l}\text { Infused in strong } \\
\text { alcohol }\end{array}$ & 4 & \\
\hline \multirow{2}{*}{$\begin{array}{l}\text { Humulus lupulus L. } \\
\text { (Cannabaceae) } \\
\text { SB081 } \\
\text { NB182 }\end{array}$} & \multirow[t]{2}{*}{ Hamei; хміль (Khmil) } & \multirow[t]{2}{*}{ Flowers } & Beer & \multirow[t]{2}{*}{6} & \\
\hline & & & Bread starter & & 2 \\
\hline $\begin{array}{l}\text { Hypericum spp. including Hypericum perforatum L. } \\
\text { (Hypericaceae) } \\
\text { SB068; SB092 } \\
\text { NB005, NB034, NB046, NB085 }\end{array}$ & $\begin{array}{l}\text { Pojarniță; Sunătoare; звіробій; } \\
\text { звіробой (Zvirobii; zviroboi) }\end{array}$ & Aerial parts & Tea & 3 & 8 \\
\hline Levisticum officinale W.D.J.Koch (Apiaceae) & Любисток (Liubystok) & Aerial parts & Tea & & 2 \\
\hline $\begin{array}{l}\text { Matricaria chamomilla L. (Asteraceae) } \\
\text { SB002; SB019 } \\
\text { NB127 }\end{array}$ & $\begin{array}{l}\text { Mușețel; Romaniț̆a ромашка; } \\
\text { романіца; романець; румєниць } \\
\text { (Romashka; romanitsa; romanets; } \\
\text { rumienyts) }\end{array}$ & Aerial parts & Tea & 4 & 9 \\
\hline $\begin{array}{l}\text { Mentha spp. } \\
\text { (Lamiaceae) } \\
\text { SB014; SB016; SB034; SB096 } \\
\text { NB079, NB080, NB097 }\end{array}$ & $\begin{array}{l}\text { Mentă de doi culoari; менти; Mentă } \\
\text { tare; Minta; мята (Miata) }\end{array}$ & Leaves & Tea & 5 & 1 \\
\hline \multirow{2}{*}{$\begin{array}{l}\text { Origanum vulgare L. } \\
\text { (Lamiaceae) } \\
\text { NB033; NB055; NB021 }\end{array}$} & \multirow[t]{2}{*}{ Материнка (Materynka) } & \multirow[t]{2}{*}{ Aerial parts } & Tea & & 4 \\
\hline & & & Seasoning & & 1 \\
\hline \multirow{2}{*}{$\begin{array}{l}\text { Oxalis acetosella L. } \\
\text { (Oxalidaceae) } \\
\text { NB058 }\end{array}$} & \multirow{2}{*}{$\begin{array}{l}\text { Квасениця звичайна (Kvasenytsia } \\
\text { zvychaina) }\end{array}$} & \multirow[t]{2}{*}{ Leaves } & Salad & & 1 \\
\hline & & & Snack & & 1 \\
\hline $\begin{array}{l}\text { Papaver rhoeas L. } \\
\text { SB044a; SB044b; SB044C }\end{array}$ & Mac & Seeds & Food additive & 1 & \\
\hline \multirow{2}{*}{$\begin{array}{l}\text { Picea abies (L.) H. Karst. } \\
\text { (Pinaceae) } \\
\text { SB008; SB021 } \\
\text { NB043 }\end{array}$} & \multirow[t]{2}{*}{ Brad; смерека (Smereka) } & Sprouts & Jam & 2 & \\
\hline & & Wood & Smoking (meat) & 1 & 2 \\
\hline $\begin{array}{l}\text { Plantago major L. } \\
\text { (Plantaginaceae) } \\
\text { NB022; NB047; NB132 }\end{array}$ & Подорожник (Podorozhnyk) & Aerial parts & Tea & & 2 \\
\hline Populus tremula L. & Осика (Osyka) & Wood & Smoking (meat) & & 1 \\
\hline
\end{tabular}

(Salicaceae) 
Table 1 Recorded food taxa in Northern and Southern Bukovina. DUR Detailed Use Reports; RO Romanian Hutsuls; UA Ukrainian Hutsuls (Continued)

\begin{tabular}{|c|c|c|c|c|c|}
\hline \multirow[t]{2}{*}{ Latin name, Family and voucher specimens } & \multirow[t]{2}{*}{ Local names } & \multirow[t]{2}{*}{ Used part(s) } & \multirow[t]{2}{*}{ Preparation } & \multicolumn{2}{|c|}{ DUR } \\
\hline & & & & $\mathrm{RO}$ & UA \\
\hline $\begin{array}{l}\text { Primula veris } \mathrm{L} \text {. } \\
\text { (Primulaceae) }\end{array}$ & Cioboțica cucului & Aerial parts & Tea & 6 & \\
\hline \multirow{2}{*}{$\begin{array}{l}\text { Quercus spp. including } \\
\text { Quercus robur L. and Quercus rubra L. } \\
\text { (Fagaceae) } \\
\text { SB056 } \\
\text { NB160 }\end{array}$} & \multirow{2}{*}{$\begin{array}{l}\text { Stejar; Duba; дуб } \\
\text { (Dub) }\end{array}$} & Leaves & Pickles (cucumbers) & & 3 \\
\hline & & $\begin{array}{l}\text { Young } \\
\text { branches }\end{array}$ & Pickles & 6 & \\
\hline $\begin{array}{l}\text { Rosa canina L. } \\
\text { (Rosaceae) } \\
\text { SB062 } \\
\text { NB016; NB083 }\end{array}$ & Măceș & Fruits & Tea & 1 & \\
\hline \multirow{4}{*}{$\begin{array}{l}\text { Rosa rugosa L.; Rosa centifolia L. (Rosaceae) } \\
\text { SB023 }\end{array}$} & \multirow[t]{4}{*}{ Trandafir; роза (Roza) } & \multirow[t]{4}{*}{ Petals } & Jam & 14 & \\
\hline & & & Jelly & 2 & \\
\hline & & & Syrup & 4 & \\
\hline & & & Tea & 4 & \\
\hline \multirow{8}{*}{$\begin{array}{l}\text { Rubus spp. including } \\
\text { Rubus caesius L. and Rubus fructicosus L. } \\
\text { (Rosaceae) } \\
\text { SB083 } \\
\text { NB010; NB062;NB063 }\end{array}$} & \multirow{8}{*}{$\begin{array}{l}\text { Чорниця; ожина; єжевіка } \\
\text { (Chornytsia; ozhyna; yezhevika) } \\
\text { Mure; чорниці (Chornytsi) }\end{array}$} & \multirow[t]{6}{*}{ Fruits } & Jam & 9 & 3 \\
\hline & & & Raw & 2 & \\
\hline & & & Compote & 1 & 1 \\
\hline & & & Infused in alcohol & 2 & \\
\hline & & & Juice & 1 & 1 \\
\hline & & & Syrup & 2 & \\
\hline & & Aerial parts & Tea & 2 & \\
\hline & & Flowers & Tea & & 1 \\
\hline \multirow{9}{*}{$\begin{array}{l}\text { Rubus idaeus L. } \\
\text { (Rosaceae) } \\
\text { SB009; SB071 } \\
\text { NB081 }\end{array}$} & \multirow{9}{*}{$\begin{array}{l}\text { Zmeură; малина } \\
\text { (Malyna) }\end{array}$} & Aerial parts & Tea & 3 & 4 \\
\hline & & Fruits & Juice & 5 & 6 \\
\hline & & & Raw & 7 & 2 \\
\hline & & & Compote & 6 & 7 \\
\hline & & & Jam & 18 & 13 \\
\hline & & & Dessert & 1 & \\
\hline & & & Syrup & 4 & \\
\hline & & & Frozen & & 2 \\
\hline & & & Tincture & & 1 \\
\hline \multirow{3}{*}{$\begin{array}{l}\text { Rumex acetosa L. } \\
\text { (Polygonaceae) } \\
\text { NB081 }\end{array}$} & \multirow{3}{*}{$\begin{array}{l}\text { Квас§; щавель; квасок§ } \\
\text { (kvas§; shchavel; kvasok§) }\end{array}$} & \multirow[t]{3}{*}{ Leaves } & Soup & & 21 \\
\hline & & & Salad & & 2 \\
\hline & & & Snack & & 2 \\
\hline $\begin{array}{l}\text { Rumex alpinus L. } \\
\text { (Polygonaceae) } \\
\text { SB067 } \\
\text { NB003 }\end{array}$ & Ştevie & Leaves & Stewed (with cream) & 1 & \\
\hline \multirow{3}{*}{$\begin{array}{l}\text { Sambucus nigra L. } \\
\text { (Adoxaceae) } \\
\text { SB084 } \\
\text { NB054 }\end{array}$} & \multirow[t]{3}{*}{ Sос; бузина ( Buzyna) } & \multirow[t]{2}{*}{ Flowers } & Juice & 2 & \\
\hline & & & Tea & 1 & \\
\hline & & Fruits & Jam & & 2 \\
\hline \multirow{3}{*}{$\begin{array}{l}\text { Sorbus spp. including Sorbus aucuparia (Rosaceae) } \\
\text { SB055 } \\
\text { NB232 }\end{array}$} & \multirow[t]{3}{*}{ Scoruş; щкорох§ (shchkorokh§) } & Flowers & Tea & & 1 \\
\hline & & Fruits & Kvas & & 1 \\
\hline & & & Various & 4 & \\
\hline Taraxacum officinale Webb (Asteraceae) & Papădie; кульбаба (kulbaba) & Flowers & Jam & 1 & \\
\hline
\end{tabular}


Table 1 Recorded food taxa in Northern and Southern Bukovina. DUR Detailed Use Reports; RO Romanian Hutsuls; UA Ukrainian Hutsuls (Continued)

\begin{tabular}{|c|c|c|c|c|c|}
\hline \multirow[t]{2}{*}{ Latin name, Family and voucher specimens } & \multirow[t]{2}{*}{ Local names } & \multirow[t]{2}{*}{ Used part(s) } & \multirow[t]{2}{*}{ Preparation } & \multicolumn{2}{|c|}{ DUR } \\
\hline & & & & $\mathrm{RO}$ & $\mathrm{UA}$ \\
\hline \multirow[t]{4}{*}{ NB016; NB048 } & & Aerial parts & Salad & & 3 \\
\hline & & Roots & Salad & & 2 \\
\hline & & & Coffee & & $2^{a}$ \\
\hline & & & Tea & & 1 \\
\hline \multirow{2}{*}{$\begin{array}{l}\text { Thymus spp. including Thymus vulgaris L. and Thymus } \\
\text { serpyllum L. } \\
\text { (Lamiaceae) } \\
\text { SB001; SB090 } \\
\text { NB027; NB125; NB030 }\end{array}$} & \multirow[b]{2}{*}{$\begin{array}{l}\text { чабер; чебрець;чебрик; городній } \\
\text { чебрець (Chaber; chebrets; chebryk; } \\
\text { horodnii chebrets ) } \\
\text { Thymus vulgaris: Cimbru; Cimbru } \\
\text { sălbatic; чеберецьсадовий } \\
\text { (cheberets sadovyi) } \\
\text { Thymus serpyllum: } \\
\text { Cimbrișor; чебрек польовий; } \\
\text { чебрець звичайний, чебрик } \\
\text { польовий; польовий чебрець } \\
\text { (chebrek polovyi; chebrets } \\
\text { zvychainyi, chebryk polovyi; } \\
\text { polovyi chebrets) }\end{array}$} & Aerial parts & Tea & 4 & 8 \\
\hline & & & Seasoning & 16 & 8 \\
\hline $\begin{array}{l}\text { Tilia cordata Mill. } \\
\text { (Malvaceae) } \\
\text { SB017 } \\
\text { NB253 }\end{array}$ & Теі; липа (Lypa) & Flowers & Tea & 2 & 12 \\
\hline \multirow{2}{*}{$\begin{array}{l}\text { Tussilago farfara L. } \\
\text { (Asteraceae) } \\
\text { SB065; SB085 } \\
\text { NB072; NB133 }\end{array}$} & \multirow{2}{*}{$\begin{array}{l}\text { Podbal; мати й мачуха (Maty у } \\
\text { machukha) }\end{array}$} & Leaves & Sarmale & 5 & \\
\hline & & Aerial parts & Tea & & 2 \\
\hline \multirow{5}{*}{$\begin{array}{l}\text { Urtica dioica L. } \\
\text { (Urticaceae) } \\
\text { SB088, } \\
\text { NB026; NB048 }\end{array}$} & \multirow[t]{5}{*}{ Urzică; кропива (Kropyva) } & \multirow{5}{*}{$\begin{array}{l}\text { Aerial parts } \\
\text { (young) }\end{array}$} & Soup & 17 & \\
\hline & & & Borsh & 4 & 25 \\
\hline & & & Stewed (with cream) & 2 & \\
\hline & & & Salad & & 1 \\
\hline & & & Seasoning & & 1 \\
\hline \multirow{15}{*}{$\begin{array}{l}\text { Vaccinium myrtillus L. } \\
\text { (Ericaceae) } \\
\text { SB006 } \\
\text { NB060 }\end{array}$} & \multirow[t]{15}{*}{$\begin{array}{l}\text { Afina; aфини; афинник } \\
\text { (Afyny; afynnyk) }\end{array}$} & \multirow[t]{2}{*}{ Aerial parts } & $\begin{array}{l}\text { Strong alcohol } \\
\text { (afinata) }\end{array}$ & 6 & \\
\hline & & & Tea & 9 & 7 \\
\hline & & \multirow[t]{13}{*}{ Fruits } & Juice & 5 & 2 \\
\hline & & & Syrup & 3 & 3 \\
\hline & & & Frozen & 1 & 2 \\
\hline & & & Preserved in rachiu & 1 & \\
\hline & & & Raw (with sugar) & 2 & 1 \\
\hline & & & Compote & 4 & 4 \\
\hline & & & Jam & 17 & 18 \\
\hline & & & Cake & 1 & \\
\hline & & & Preserved with sugar & 1 & \\
\hline & & & 'Wine' & 1 & 1 \\
\hline & & & Dessert & & 3 \\
\hline & & & Dried & & 1 \\
\hline & & & Snack & & 1 \\
\hline \multirow{3}{*}{$\begin{array}{l}\text { Vaccinium vitis-idaea L. } \\
\text { (Ericaceae) } \\
\text { SB010 } \\
\text { NB061 }\end{array}$} & \multirow{3}{*}{ 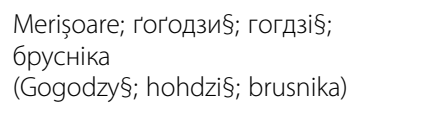 } & \multirow[t]{3}{*}{ Fruits } & Raw & 4 & \\
\hline & & & Jam & 7 & 9 \\
\hline & & & Juice & 8 & 1 \\
\hline
\end{tabular}


Table 1 Recorded food taxa in Northern and Southern Bukovina. DUR Detailed Use Reports; RO Romanian Hutsuls; UA Ukrainian Hutsuls (Continued)

\begin{tabular}{|c|c|c|c|c|c|}
\hline \multirow[t]{2}{*}{ Latin name, Family and voucher specimens } & \multirow[t]{2}{*}{ Local names } & \multirow[t]{2}{*}{ Used part(s) } & \multirow[t]{2}{*}{ Preparation } & \multicolumn{2}{|c|}{ DUR } \\
\hline & & & & $\overline{\mathrm{RO}}$ & $\overline{U A}$ \\
\hline & & & Drink & 1 & \\
\hline & & & Compote & 2 & 1 \\
\hline & & & Syrup & & 2 \\
\hline & & & Frozen & & 1 \\
\hline & & & Snack & & 1 \\
\hline & & & Tea & & 3 \\
\hline & & & Kvas & & 1 \\
\hline \multirow{4}{*}{$\begin{array}{l}\text { Viburnum opulus L. } \\
\text { (Adoxaceae) } \\
\text { NB223 }\end{array}$} & Călină; калина (Kalyna) & Fruits & $\begin{array}{l}\text { Strong alcohol } \\
\text { (Calinata) }\end{array}$ & 4 & \\
\hline & & & Preserved in jars & 2 & \\
\hline & & & Syrup & 2 & \\
\hline & & Aerial parts & Tea & & 3 \\
\hline
\end{tabular}

Plant names mentioned by Ukrainian Hutsuls are reported in Cyrillic (with transliteration). Plant names mentioned by Romanian Hutsuls are reported in the Latin alphabet. Plant names not reported in Romanian or Ukrainian dictionaries or in publications available for the area (e.g. Pieroni and Soukand, 2017), and are therefore probably Hutsul names, are marked with a §. Russian names are marked with a $\wedge$

${ }^{a}$ denotes a past use

also reported) and Quercus spp. (young branches in Romania and leaves in Ukraine), and Carum carvi only in Ukraine. Many other cultivated plants (cucumbers, carrots, garlic, cabbage, cauliflower, as well as mushrooms in some cases) were added to this preparation, which is later fermented. Another peculiar mode of preparation reported in Ukraine is 'KBac (kvas)', a drink made from fermented grain and low in alcohol content. Birch sap was also reported as an ingredient for kvas. Such a drink is often flavoured with berries and fruits, including Aronia melanocarpa, Sorbus spp. and Vaccinium vitis-idaea which were mentioned by interviewees.

On both sides of the border, berries were often prepared as compote, which is made by boiling fruits (in this case Fragaria vesca, Rubus idaeus, Rubus caesius, Vaccinium myrtillus, Vaccinium vitis-idaea) in abundant water and later removing them to drink the flavoured liquid. Berries are either eaten as a dessert or thrown away. The compote can be prepared with or without adding sugar (e.g. Vaccinium myrtillus compote). Compote was often reported as a preserve for winter time.

Freezing as a conservation method was mentioned only by one person in Romania (for Vaccinium myrtillus), while it was more often reported in Ukraine for other berries (Rubus idaeus, Fragaria vesca and Vaccinium vitis-idaea).

\section{Medicinal taxa}

We recorded 111 plant taxa used for medicinal purposes (Table 2). Specifically, 64 taxa were used among Romanian Hutsuls while 100 were used among Ukrainian
Hutsuls, with 53 taxa shared in common (Fig. 5). This disparity was also reflected in the number of DURs: 840 in Northern Bukovina and 585 in Southern Bukovina $(-30 \%)$. This trend was also reported by Sõukand and Pieroni [16]. The Jaccard Similarity Index did not vary much when considering all taxa (48) or only those mentioned by at least $10 \%$ of the interviewees (46).

The most common medicinal taxon was the same in both communities, namely Vaccinium myrtillus (78 DUR among Ukrainian Hutsuls and 45 DUR among Romanian Hutsuls). In Northern Bukovina, it was followed by Rubus idaeus (46 DUR), Urtica dioica (32 DUR), Plantago major (31 DUR) and Vaccinium vitis-idaea (27 DUR). In Southern Bukovina, it was followed by Urtica dioica (35 DUR), Hypericum spp. (33 DUR), Tilia spp. (32 DUR) and Rubus idaeus (27 DUR). Half of the reported medicinal DURs on both sides of the border are for cultivated plants, while wild species represent $24 \%$ and $31 \%$ of the reported taxa in Northern and Southern Bukovina, respectively.

Romanian Hutsuls particularly mentioned medicinal taxa for treating the respiratory system, the digestive system and for general health (Fig. 6). In the first two cases, they reported more DURs than did Ukrainian Hutsuls. In Northern Bukovina, the first three medicinal categories reported by Hutsul interviewees were general health, the respiratory system and the digestive system.

Following the higher number of medicinal DUR among Ukrainian Hutsuls, they exceeded Romanian Hutsuls in all medicinal categories apart from those of the auditory, digestive and respiratory systems. Interestingly, cancer (neoplasm category, four taxa) was only mentioned in Ukraine, 
a.

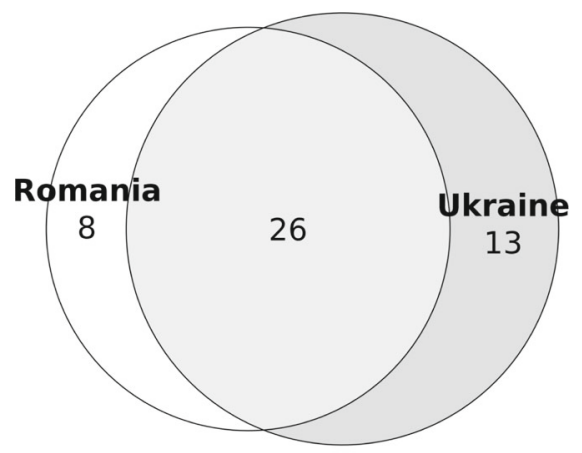

b.

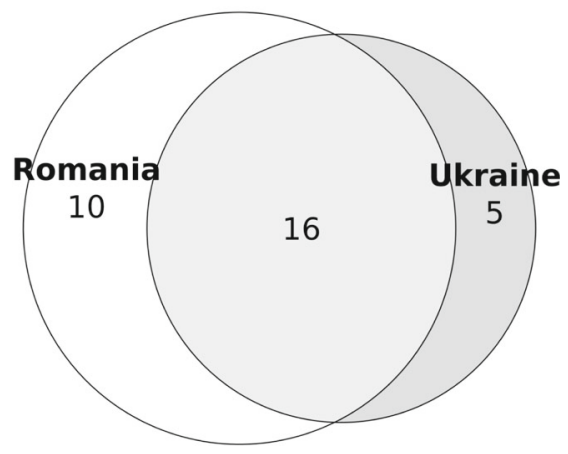

Fig. 4 a The proportional Venn diagram shows that most of the food taxa mentioned are common to Hutsul communities of Northern and Southern Bukovina; $\mathbf{l}=55$. $\mathbf{b}$ The proportional Venn diagram of food taxa mentioned by at least three interviewees shows that Romanian Hutsuls reported more consistent uses than Ukrainian Hutsuls. Indeed, several food taxa were mentioned by only one or two Ukrainian Hutsuls; $J=51$

whereas in Romania two people reported a remedy for cancer using Helleborus foetidus, but then added that they do not to use it as it is very dangerous.

We recorded a total of 128 emic medicinal categories which were nearly equally distributed: 42 were reported by both communities, 41 among Romanian Hutsuls and 45 among Ukrainian Hutsuls.

Only ten medicinal DURs used by at least $10 \%$ of each community were found on both sides of the border. Three DURs were included in the digestive category and specifically considered as good for the stomach: tea made from the seeds of Carum carvi (used by one fifth of the interviewees), dried aerial parts of Hypericum perforatum and any preparation of Vaccinium myrtillus. Two musculoskeletal remedies include compresses of the leaves of Arctium lappa and the flowers of Arnica montana infused in alcohol, locally applied to treat joint pain. The aerial parts of Rubus idaeus are prepared as tea to reduce fever, while the aerial parts of Urtica dioica are boiled and used to wash the hair (for strong and shiny hair). More than $30 \%$ of both communities consider Thymus spp. as a remedy for cough. Finally, the fresh leaves of Plantago major are locally applied to warts and the young sprouts of Urtica dioica are considered beneficial for cleansing the blood.

\section{Knowledge transmission}

We recorded eight sources of knowledge among both Romanian and Ukrainian Hutsuls. Three categories differ between the two groups: friends, professors and a local healer (in the past) were mentioned in Southern Bukovina, while television, the Internet and newspapers were mentioned in Northern Bukovina. When analysing these data in the framework of the abovementioned Van den Boog [10] study, we observed that in $45 \%$ of cases Romanian Hutsuls transferred their knowledge vertically (from parents, grandparents and great-grandparents), $42 \%$ obliquely (via the elderly of the village) and $4 \%$ horizontally (through friends and neighbours), while $4 \%$ received knowledge from specialists (local healers and professors) and written sources (books) accounted for 2\% (Fig. 7). Among the books, one elderly interviewee mentioned Maria Treben's [29] bestseller (for the preparation of Primula tea), but most of the Romanian Hutsuls said they did not have time for reading as 
Table 2 Recorded medicinal taxa in Northern and Southern Bukovina. DUR Detailed Use Reports; RO Romanian Hutsuls; UA Ukrainian Hutsuls

\begin{tabular}{|c|c|c|c|c|c|c|}
\hline \multirow{2}{*}{$\begin{array}{l}\text { Latin name, family and voucher } \\
\text { specimens }\end{array}$} & \multirow[t]{2}{*}{ Local names } & \multirow[t]{2}{*}{ Used part(s) } & \multirow[t]{2}{*}{ Preparation } & \multirow[t]{2}{*}{ Medicinal Use } & \multicolumn{2}{|c|}{ DUR } \\
\hline & & & & & $\overline{\mathrm{RO}}$ & $\overline{U A}$ \\
\hline \multirow{4}{*}{$\begin{array}{l}\text { Abies alba Mill. possibly including } \\
\text { Picea abies (L.) H. Karst. } \\
\text { (Pinaceae) }\end{array}$} & \multirow[t]{4}{*}{ Molid; ялина (Yalyna) } & Resin & Locally Applied & Joint pain & & 2 \\
\hline & & Young sprouts & Syrup (fresh) & Fever & 1 & \\
\hline & & & & Cough & 2 & \\
\hline & & & & $\begin{array}{l}\text { Good for } \\
\text { lungs }\end{array}$ & 5 & \\
\hline \multirow[t]{2}{*}{$\begin{array}{l}\text { Achillea carpatica Blocki ex } \\
\text { Dubovik } \\
\text { (Asteraceae) }\end{array}$} & \multirow[t]{2}{*}{ Деревій карпатський (Derevii karpatskyi) } & Aerial parts & Tea (dried) & $\begin{array}{l}\text { Digestive } \\
\text { system } \\
\text { problems }\end{array}$ & & 1 \\
\hline & & & & $\begin{array}{l}\text { Stomach } \\
\text { diseases }\end{array}$ & & 1 \\
\hline \multirow{16}{*}{$\begin{array}{l}\text { Achillea millefolium L. (Asteraceae) } \\
\text { SB011; SB074; SB050 } \\
\text { NB007; NB017; NB039 }\end{array}$} & \multirow{16}{*}{$\begin{array}{l}\text { Coada șoaricelului; деревій; деревій, } \\
\text { тисячолітник; деревій звичайний; } \\
\text { деревій буковинський } \\
\text { (Derevii; derevii, tysiacholitnyk; } \\
\text { derevii zvychainyi; derevii bukovynskyi) }\end{array}$} & \multirow[t]{16}{*}{ Aerial parts } & Tea & $\begin{array}{l}\text { Vessel } \\
\text { cleansing }\end{array}$ & & 1 \\
\hline & & & $\begin{array}{l}\text { Locally applied } \\
\text { (juice of } \\
\text { pressed leaves) }\end{array}$ & Wounds & & 3 \\
\hline & & & \multirow[t]{6}{*}{ Tea } & Diarrhoea & & 3 \\
\hline & & & & $\begin{array}{l}\text { Digestive } \\
\text { system } \\
\text { problems }\end{array}$ & & 1 \\
\hline & & & & $\begin{array}{l}\text { Good for the } \\
\text { liver }\end{array}$ & 1 & \\
\hline & & & & $\begin{array}{l}\text { Good for the } \\
\text { stomach }\end{array}$ & 2 & 5 \\
\hline & & & & Vomiting & & 1 \\
\hline & & & & Disinfectant & 4 & \\
\hline & & & $\begin{array}{l}\text { Tea with } \\
\text { Chelidonium }\end{array}$ & Disinfectant & 4 & \\
\hline & & & \multirow[t]{7}{*}{ Tea } & Hair care & 4 & \\
\hline & & & & Pain & & 1 \\
\hline & & & & Panacea & & 1 \\
\hline & & & & Aching legs & & 1 \\
\hline & & & & Calming & & 2 \\
\hline & & & & Toothache & & 3 \\
\hline & & & & Cold & 2 & \\
\hline \multirow{2}{*}{$\begin{array}{l}\text { Acorus calamus L. (Acoraceae) } \\
\text { NB121 }\end{array}$} & \multirow[t]{2}{*}{ Aïp (Air) } & \multirow[t]{2}{*}{ Roots } & \multirow[t]{2}{*}{ Tea } & Diarrhoea & & 1 \\
\hline & & & & $\begin{array}{l}\text { Good for the } \\
\text { stomach }\end{array}$ & & 1 \\
\hline \multirow{5}{*}{$\begin{array}{l}\text { Aesculus hippocastanum L. } \\
\text { (Sapindaceae) } \\
\text { SB057 } \\
\text { NB067 }\end{array}$} & \multirow{5}{*}{$\begin{array}{l}\text { Castan; каштан; каштан } \\
\text { кінський червоний; каштан } \\
\text { чеворний (Kashtan; kashtan } \\
\text { kinskyi chervonyi; kashtan } \\
\text { chevornyi) }\end{array}$} & \multirow[t]{2}{*}{ Flowers } & \multirow{2}{*}{$\begin{array}{l}\text { Locally applied } \\
\text { (in alcohol/ } \\
\text { moonshine) }\end{array}$} & Feet pain & & 1 \\
\hline & & & & Joint pain & 1 & 7 \\
\hline & & Fruits & $\begin{array}{l}\text { Infused in } \\
\text { alcohol }\end{array}$ & $\begin{array}{l}\text { Good for } \\
\text { blood vessels }\end{array}$ & & 1 \\
\hline & & & \multirow{2}{*}{$\begin{array}{l}\text { Locally applied } \\
\text { (in alcohol/ } \\
\text { moonshine) }\end{array}$} & Foot pain & & 1 \\
\hline & & & & Joint pain & & 5 \\
\hline $\begin{array}{l}\text { Alchemilla vulgaris auct. } \\
\text { (coll.) } \\
\text { (Rosaceae) } \\
\text { SB039 }\end{array}$ & Crețișoara; Гарник (Harnyk) & Aerial parts & $\begin{array}{l}\text { Locally applied } \\
\text { (infused in } \\
\text { alcohol) }\end{array}$ & Joint pain & 1 & 2 \\
\hline \multirow{2}{*}{$\begin{array}{l}\text { Allium cepa L. } \\
\text { (Amaryllidaceae) }\end{array}$} & \multirow{2}{*}{$\begin{array}{l}\text { Ceapă; цибулька; цибуля } \\
\text { (Tsybulka; tsybulia) }\end{array}$} & \multirow[t]{2}{*}{ Bulbs } & \multirow[t]{2}{*}{ Raw } & Blood pressure & & 1 \\
\hline & & & & Iron & & 1 \\
\hline
\end{tabular}


Table 2 Recorded medicinal taxa in Northern and Southern Bukovina. DUR Detailed Use Reports; RO Romanian Hutsuls; UA Ukrainian Hutsuls (Continued)

\begin{tabular}{|c|c|c|c|c|c|c|}
\hline \multirow{2}{*}{$\begin{array}{l}\text { Latin name, family and voucher } \\
\text { specimens }\end{array}$} & \multirow[t]{2}{*}{ Local names } & \multirow[t]{2}{*}{ Used part(s) } & \multirow[t]{2}{*}{ Preparation } & \multirow[t]{2}{*}{ Medicinal Use } & \multicolumn{2}{|c|}{ DUR } \\
\hline & & & & & $\mathrm{RO}$ & UA \\
\hline & & & & Flu & & 2 \\
\hline & & & Tea & Fever & 1 & \\
\hline & & & & Cough & 2 & \\
\hline & & & & $\begin{array}{l}\text { Good for the } \\
\text { lungs }\end{array}$ & 1 & \\
\hline & & & Boiled & Cough & 1 & 3 \\
\hline & & & $\begin{array}{l}\text { Raw (with } \\
\text { honey and } \\
\text { sugar) }\end{array}$ & Cough & 1 & \\
\hline & & External shell & Fomentation & $\begin{array}{l}\text { Women's } \\
\text { problems }\end{array}$ & & 1 \\
\hline \multirow[t]{7}{*}{$\begin{array}{l}\text { Allium sativum L. (Amaryllidaceae) } \\
\text { NB192 }\end{array}$} & \multirow[t]{7}{*}{ Usturoi; часник (Chasnyk) } & Bulbs & Raw & $\begin{array}{l}\text { Blood } \\
\text { cleansing }\end{array}$ & & 1 \\
\hline & & & & Cancer & & 1 \\
\hline & & & & $\begin{array}{l}\text { Immune } \\
\text { boosting }\end{array}$ & & 1 \\
\hline & & & & Vitamins & & 1 \\
\hline & & & Raw & Flu & & 2 \\
\hline & & & Locally applied & Earache & 2 & \\
\hline & & & $\begin{array}{l}\text { Crushed and } \\
\text { locally applied } \\
\text { with massage }\end{array}$ & Flu & & 2 \\
\hline $\begin{array}{l}\text { Alnus glutinosa (L.) Gaertn. } \\
\text { (Betulaceae) } \\
\text { NB050; NB052 }\end{array}$ & Дубило§; вільха (Dubylo§; vilkha) & Bark & Boiled & Gangrene & & 2 \\
\hline \multirow{2}{*}{$\begin{array}{l}\text { Aloe vera (L.) Burm.f. } \\
\text { (Xanthorrhoeaceae) }\end{array}$} & \multirow[t]{2}{*}{ Алое (Aloe) } & Leaves & Locally applied & Wounds & & 3 \\
\hline & & & Raw & Abscesses & & 1 \\
\hline \multirow{6}{*}{$\begin{array}{l}\text { Anethum graveolens L. } \\
\text { (Apiaceae) } \\
\text { SB032 }\end{array}$} & \multirow[t]{6}{*}{ Кріп (Krip) } & $\begin{array}{l}\text { Aerial parts, } \\
\text { seeds }\end{array}$ & Tea & Panacea & & 1 \\
\hline & & Leaves & Raw & Vitamins & & 1 \\
\hline & & Seeds & Tea & Blood pressure & & 2 \\
\hline & & & & $\begin{array}{l}\text { Good for the } \\
\text { stomach }\end{array}$ & & 2 \\
\hline & & & & Fever & & 2 \\
\hline & & & Dried & $\begin{array}{l}\text { To increase } \\
\text { milk } \\
\text { production in } \\
\text { women }\end{array}$ & & 3 \\
\hline \multirow{4}{*}{$\begin{array}{l}\text { Arctium lappa L. } \\
\text { (Asteraceae) } \\
\text { SB052; SB091 } \\
\text { NB149 }\end{array}$} & \multirow{4}{*}{$\begin{array}{l}\text { Brusturi; Brusturoi; лопух; рипях; } \\
\text { лопух; рипяка (Lopukh; rypiakh; } \\
\text { rypiaka) }\end{array}$} & Flowers & Boiled & Hair care & & 2 \\
\hline & & Leaves & $\begin{array}{l}\text { Locally applied } \\
\text { (fresh and } \\
\text { crushed) }\end{array}$ & Joint pain & 3 & 3 \\
\hline & & & Raw & Headache & & 2 \\
\hline & & Roots & Boiled & Hair care & & 9 \\
\hline \multirow{3}{*}{$\begin{array}{l}\text { Armoracia rusticana P.Gaertn., B.Mey. \& } \\
\text { Scherb. } \\
\text { (Brassicaceae) } \\
\text { SB031 } \\
\text { NB028; NB212 }\end{array}$} & \multirow[t]{3}{*}{$\begin{array}{l}\text { Hrean; хрін; хреню } \\
\text { (Khrin; khreniu) }\end{array}$} & Leaves & $\begin{array}{l}\text { Locally applied } \\
\text { on the head } \\
\text { (fresh) }\end{array}$ & Fever & 1 & \\
\hline & & & Locally applied & Joint pain & & 1 \\
\hline & & Roots & Raw (in food) & Help the & 1 & \\
\hline
\end{tabular}


Table 2 Recorded medicinal taxa in Northern and Southern Bukovina. DUR Detailed Use Reports; RO Romanian Hutsuls; UA Ukrainian Hutsuls (Continued)

\begin{tabular}{|c|c|c|c|c|c|c|}
\hline \multirow{2}{*}{$\begin{array}{l}\text { Latin name, family and voucher } \\
\text { specimens }\end{array}$} & \multirow[t]{2}{*}{ Local names } & \multirow[t]{2}{*}{ Used part(s) } & \multirow[t]{2}{*}{ Preparation } & \multirow[t]{2}{*}{ Medicinal Use } & \multicolumn{2}{|c|}{ DUR } \\
\hline & & & & & $\mathrm{RO}$ & UA \\
\hline & & & & bloodstream & & \\
\hline & & & & Healthy & 1 & \\
\hline & & & Locally applied & Joint pain & & 1 \\
\hline & & & & $\begin{array}{l}\text { Rheumatic } \\
\text { pains }\end{array}$ & & 1 \\
\hline & & & Raw (in food) & $\begin{array}{l}\text { Opening } \\
\text { airways }\end{array}$ & 1 & \\
\hline \multirow[t]{15}{*}{$\begin{array}{l}\text { Arnica montana L. } \\
\text { (Asteraceae) }\end{array}$} & \multirow[t]{15}{*}{ Arnică; Arnic; Harnic; apнiкa (Arnica) } & \multirow[t]{15}{*}{ Flowers } & \multirow[t]{2}{*}{ Tea } & $\begin{array}{l}\text { Good for the } \\
\text { heart }\end{array}$ & & 4 \\
\hline & & & & $\begin{array}{l}\text { Good for the } \\
\text { eyes }\end{array}$ & & 1 \\
\hline & & & \multirow{8}{*}{$\begin{array}{l}\text { Locally applied } \\
\text { (infused in } \\
\text { alcohol) }\end{array}$} & Heart diseases & & 1 \\
\hline & & & & Aching legs & & 2 \\
\hline & & & & Back pain & 1 & \\
\hline & & & & Foot pain & 2 & \\
\hline & & & & Hand pain & 2 & \\
\hline & & & & Joint pain & 4 & 8 \\
\hline & & & & $\begin{array}{l}\text { Rheumatic } \\
\text { pains }\end{array}$ & 5 & 1 \\
\hline & & & & Wrist pain & 1 & \\
\hline & & & \multirow{2}{*}{$\begin{array}{l}\text { Locally applied } \\
\text { with (olive) oil }\end{array}$} & Hand pain & 2 & \\
\hline & & & & Joint pain & & 2 \\
\hline & & & \multirow{2}{*}{$\begin{array}{l}\text { Infused in } \\
\text { alcohol (fresh) }\end{array}$} & Panacea & $1^{\mathrm{b}}$ & \\
\hline & & & & $\begin{array}{l}\text { Good for the } \\
\text { skin }\end{array}$ & $1^{\mathrm{b}}$ & \\
\hline & & & $\begin{array}{l}\text { Bath (fresh/ } \\
\text { dried) }\end{array}$ & Foot pain & 1 & \\
\hline \multirow{2}{*}{$\begin{array}{l}\text { Aronia melanocarpa (Michx.) } \\
\text { Elliott } \\
\text { (Rosaceae) }\end{array}$} & \multirow{2}{*}{$\begin{array}{l}\text { горобина чорна; шкорух§; } \\
\text { чорна горобина; рябина } \\
\text { (Horobyna chorna; shkorukh§; } \\
\text { chorna; horobyna; riabyna) }\end{array}$} & \multirow[t]{2}{*}{ Fruits } & Tea & Blood pressure & & 7 \\
\hline & & & Syrup & Blood pressure & & 1 \\
\hline \multirow{7}{*}{$\begin{array}{l}\text { Artemisia absinthium L. (Asteraceae) } \\
\text { SB005 } \\
\text { NB051 }\end{array}$} & \multirow{7}{*}{$\begin{array}{l}\text { Pelin; полинь; полин } \\
\text { (Polyn; polyn) }\end{array}$} & \multirow[t]{6}{*}{ Aerial parts } & \multirow[t]{3}{*}{ Tea } & Diarrhoea & & 1 \\
\hline & & & & $\begin{array}{l}\text { Good for the } \\
\text { stomach }\end{array}$ & 1 & \\
\hline & & & & Stomach pain & & 1 \\
\hline & & & $\begin{array}{l}\text { Tincture with } \\
\text { alcohol }\end{array}$ & $\begin{array}{l}\text { Appetite } \\
\text { stimulant }\end{array}$ & 2 & \\
\hline & & & \multirow[t]{2}{*}{ Tea } & Panacea & & 1 \\
\hline & & & & Cancer & & $1^{\mathrm{a}}$ \\
\hline & & Seeds & Tea & Diarrhoea & & 1 \\
\hline \multirow[t]{4}{*}{ Atropa belladonna L. (Solanaceae) } & \multirow[t]{4}{*}{ Матриган § (Matrygan) } & \multirow[t]{4}{*}{ Roots } & \multirow{4}{*}{$\begin{array}{l}\text { Infused in } \\
\text { alcohol/ } \\
\text { moonshine }\end{array}$} & $\begin{array}{l}\text { Reproductive } \\
\text { potency }\end{array}$ & & 1 \\
\hline & & & & Cancer & & 1 \\
\hline & & & & $\begin{array}{l}\text { Good for } \\
\text { women }\end{array}$ & & 1 \\
\hline & & & & Joint pain & & 3 \\
\hline Avena sativa L. & Овес (Oves) & Seeds & Tea & Healthy & & 2 \\
\hline
\end{tabular}


Table 2 Recorded medicinal taxa in Northern and Southern Bukovina. DUR Detailed Use Reports; RO Romanian Hutsuls; UA Ukrainian Hutsuls (Continued)

\begin{tabular}{|c|c|c|c|c|c|c|}
\hline \multirow{2}{*}{$\begin{array}{l}\text { Latin name, family and voucher } \\
\text { specimens }\end{array}$} & \multirow[t]{2}{*}{ Local names } & \multirow[t]{2}{*}{ Used part(s) } & \multirow[t]{2}{*}{ Preparation } & \multirow[t]{2}{*}{ Medicinal Use } & \multicolumn{2}{|c|}{ DUR } \\
\hline & & & & & $\overline{\mathrm{RO}}$ & $\overline{U A}$ \\
\hline NB202 & & & & Kidney stones & & 2 \\
\hline $\begin{array}{l}\text { Bellis perennis } \mathrm{L} \text {. } \\
\text { (Asteraceae) }\end{array}$ & Маргаритки (Marharytky) & Flowers & Raw & $\begin{array}{l}\text { Good for the } \\
\text { heart }\end{array}$ & & 2 \\
\hline \multirow{8}{*}{$\begin{array}{l}\text { Beta vulgaris L. (Amaranthaceae) } \\
\text { SB026 }\end{array}$} & \multirow{8}{*}{$\begin{array}{l}\text { Sfeclă; буряк червоний } \\
\text { (Buriak chervonyi) }\end{array}$} & \multirow[t]{8}{*}{ Tubers } & Any preparation & Anaemia & 2 & \\
\hline & & & \multirow[t]{7}{*}{ Juice } & $\begin{array}{l}\text { Blood } \\
\text { cleansing }\end{array}$ & & 2 \\
\hline & & & & $\begin{array}{l}\text { Good for } \\
\text { haemoglobin }\end{array}$ & & 2 \\
\hline & & & & Healthy & 1 & \\
\hline & & & & Joint pain & & 2 \\
\hline & & & & Headache & & 2 \\
\hline & & & & Cough & 1 & 2 \\
\hline & & & & $\begin{array}{l}\text { Good for the } \\
\text { throat }\end{array}$ & & 2 \\
\hline \multirow{13}{*}{$\begin{array}{l}\text { Betula pendula Roth } \\
\text { (Betulaceae) } \\
\text { SB087 } \\
\text { NB041; NB115 }\end{array}$} & \multirow[t]{13}{*}{ Mesteacăn; береза (Bereza) } & Bark & Boiled & Gangrene & & 2 \\
\hline & & $\begin{array}{l}\text { Buds and } \\
\text { leaves }\end{array}$ & Tea & $\begin{array}{l}\text { Blood } \\
\text { cleansing }\end{array}$ & & $1^{\mathrm{b}}$ \\
\hline & & Flowers & Tea & $\begin{array}{l}\text { Good for } \\
\text { kidneys }\end{array}$ & & 2 \\
\hline & & \multirow[t]{2}{*}{ Leaves } & Boiled & Hair care & & 2 \\
\hline & & & Tea & Healthy & & 1 \\
\hline & & \multirow[t]{7}{*}{ Sap } & \multirow[t]{7}{*}{ Drink } & $\begin{array}{l}\text { Good for the } \\
\text { heart }\end{array}$ & 1 & \\
\hline & & & & $\begin{array}{l}\text { Vascular } \\
\text { problems }\end{array}$ & 1 & \\
\hline & & & & $\begin{array}{l}\text { Good for the } \\
\text { stomach }\end{array}$ & 1 & \\
\hline & & & & Healthy & 2 & \\
\hline & & & & $\begin{array}{l}\text { Good for the } \\
\text { kidneys }\end{array}$ & 2 & 1 \\
\hline & & & & $\begin{array}{l}\text { Good for the } \\
\text { lungs }\end{array}$ & 6 & \\
\hline & & & & $\begin{array}{l}\text { Lung } \\
\text { cleansing }\end{array}$ & 4 & \\
\hline & & Young leaves & Compress & Joint pain & & 1 \\
\hline $\begin{array}{l}\text { Bidens tripartita L. (Asteraceae) } \\
\text { NB090 }\end{array}$ & Череда (Chereda) & Aerial parts & $\begin{array}{l}\text { Tea for bathing } \\
\text { kids }\end{array}$ & $\begin{array}{l}\text { Good for the } \\
\text { skin }\end{array}$ & & 1 \\
\hline \multirow[t]{6}{*}{ Brassica oleracea L. (Brassicaceae) } & \multirow[t]{6}{*}{ Varză; капуста (Kapusta) } & \multirow[t]{6}{*}{ Leaves } & \multirow[t]{3}{*}{$\begin{array}{l}\text { Fermented } \\
\text { juice }\end{array}$} & $\begin{array}{l}\text { Good for } \\
\text { cholesterol }\end{array}$ & & 2 \\
\hline & & & & $\begin{array}{l}\text { Good for } \\
\text { pancreas }\end{array}$ & & 2 \\
\hline & & & & $\begin{array}{l}\text { Good for the } \\
\text { stomach }\end{array}$ & & 1 \\
\hline & & & $\begin{array}{l}\text { Locally applied } \\
\text { (fresh) }\end{array}$ & Frostbite & 1 & \\
\hline & & & $\begin{array}{l}\text { Poultice applied } \\
\text { on the back }\end{array}$ & Fever & 1 & \\
\hline & & & $\begin{array}{l}\text { Locally applied } \\
\text { (fresh) }\end{array}$ & $\begin{array}{l}\text { Fracture } \\
\text { Joint pain }\end{array}$ & 1 & 2 \\
\hline
\end{tabular}


Table 2 Recorded medicinal taxa in Northern and Southern Bukovina. DUR Detailed Use Reports; RO Romanian Hutsuls; UA Ukrainian Hutsuls (Continued)

\begin{tabular}{|c|c|c|c|c|c|c|}
\hline \multirow{2}{*}{$\begin{array}{l}\text { Latin name, family and voucher } \\
\text { specimens }\end{array}$} & \multirow[t]{2}{*}{ Local names } & \multirow[t]{2}{*}{ Used part(s) } & \multirow[t]{2}{*}{ Preparation } & \multirow[t]{2}{*}{ Medicinal Use } & \multicolumn{2}{|c|}{ DUR } \\
\hline & & & & & $\mathrm{RO}$ & UA \\
\hline & & & & Headache & 1 & 2 \\
\hline $\begin{array}{l}\text { Bryophyllum pinnatum (Lam.) Oken } \\
\text { (Crassulaceae) }\end{array}$ & Каланхое (Kalancoe) & Sap & Drink & Rhinitis & & 2 \\
\hline \multirow{17}{*}{$\begin{array}{l}\text { Calendula officinalis L. (Asteraceae) } \\
\text { NB233 }\end{array}$} & \multirow{17}{*}{$\begin{array}{l}\text { Gălbenele; нагідки, крокіси§; } \\
\text { крокіс§; календула; нагідки } \\
\text { (Nahidky, krokisy ; krokis; } \\
\text { kalendula; nahidky) }\end{array}$} & \multirow[t]{17}{*}{ Flowers } & \multirow[t]{13}{*}{ Tea } & Blood pressure & & 1 \\
\hline & & & & Skin cleansing & 1 & \\
\hline & & & & $\begin{array}{l}\text { Good for the } \\
\text { liver }\end{array}$ & 3 & 2 \\
\hline & & & & $\begin{array}{l}\text { Good for the } \\
\text { stomach }\end{array}$ & 2 & \\
\hline & & & & 100 diseases & & 2 \\
\hline & & & & $\begin{array}{l}\text { Immune } \\
\text { boosting }\end{array}$ & & 2 \\
\hline & & & & $\begin{array}{l}\text { Inflammation } \\
\text { processes }\end{array}$ & & 1 \\
\hline & & & & $\begin{array}{l}\text { Good for } \\
\text { women }\end{array}$ & & 2 \\
\hline & & & & $\begin{array}{l}\text { Women's } \\
\text { problems }\end{array}$ & & 2 \\
\hline & & & & $\begin{array}{l}\text { Good for the } \\
\text { kidneys }\end{array}$ & & 2 \\
\hline & & & & Cough & & $1^{\mathrm{b}}$ \\
\hline & & & & Sore throat & & $1^{\mathrm{b}}$ \\
\hline & & & & $\begin{array}{l}\text { Stomatitis } \\
\text { (kids) }\end{array}$ & & $1^{\mathrm{b}}$ \\
\hline & & & \multirow{3}{*}{$\begin{array}{l}\text { Boiled with fat } \\
\text { and locally } \\
\text { applied }\end{array}$} & $\begin{array}{l}\text { Good for the } \\
\text { skin }\end{array}$ & 1 & \\
\hline & & & & Warts & 1 & \\
\hline & & & & Fever & 1 & \\
\hline & & & Syrup & Cough & 1 & \\
\hline $\begin{array}{l}\text { Callisia fragrans (Lindl.) } \\
\text { Woodson } \\
\text { (Commelinaceae) }\end{array}$ & золотий ус (zolotyi us) & Leaves & Tea & $\begin{array}{l}\text { Blood } \\
\text { cleansing }\end{array}$ & & $1^{\mathrm{a}}$ \\
\hline Cannabis sativa L. (Cannabaceae) & Cânepă & Leaves & Burnt & Ear pain & 1 & \\
\hline \multirow{3}{*}{$\begin{array}{l}\text { Capsella bursa-pastoris L. } \\
\text { (Brassicaceae) } \\
\text { NB218 }\end{array}$} & \multirow[t]{3}{*}{ Грицики (Hrytsyky) } & \multirow[t]{3}{*}{ Aerial parts } & \multirow[t]{3}{*}{ Tea } & Blood pressure & & 1 \\
\hline & & & & $\begin{array}{l}\text { Women's } \\
\text { problems }\end{array}$ & & 1 \\
\hline & & & & Headache & & 1 \\
\hline \multirow{8}{*}{$\begin{array}{l}\text { Carum carvi L. } \\
\text { (Apiaceae) } \\
\text { SB007 } \\
\text { NB037 }\end{array}$} & \multirow{8}{*}{$\begin{array}{l}\text { Săcărică; Secărică; Chimen; } \\
\text { Hmel; Chimion; хміль§ (Khmil ) }\end{array}$} & \multirow[t]{8}{*}{ Aerial parts } & \multirow[t]{8}{*}{ Tea } & Colds & 3 & \\
\hline & & & & Diarrhoea & 5 & 2 \\
\hline & & & & $\begin{array}{l}\text { Good for the } \\
\text { abdomen }\end{array}$ & 2 & \\
\hline & & & & 100 diseases & & 2 \\
\hline & & & & Healthy & & 2 \\
\hline & & & & $\begin{array}{l}\text { Strengthening } \\
\text { of the } \\
\text { organism }\end{array}$ & 1 & \\
\hline & & & & Cough & 1 & \\
\hline & & & & Good for the & 1 & \\
\hline
\end{tabular}


Table 2 Recorded medicinal taxa in Northern and Southern Bukovina. DUR Detailed Use Reports; RO Romanian Hutsuls; UA Ukrainian Hutsuls (Continued)

\begin{tabular}{|c|c|c|c|c|c|c|}
\hline \multirow{2}{*}{$\begin{array}{l}\text { Latin name, family and voucher } \\
\text { specimens }\end{array}$} & \multirow[t]{2}{*}{ Local names } & \multirow[t]{2}{*}{ Used part(s) } & \multirow[t]{2}{*}{ Preparation } & \multirow[t]{2}{*}{ Medicinal Use } & \multicolumn{2}{|c|}{ DUR } \\
\hline & & & & & $\mathrm{RO}$ & UA \\
\hline & & & & throat & & \\
\hline & & & $\begin{array}{l}\text { Infused in } \\
\text { alcohol }\end{array}$ & Hair care & & 1 \\
\hline & & Seeds & Tea & $\begin{array}{l}\text { Good for the } \\
\text { stomach }\end{array}$ & 5 & 5 \\
\hline $\begin{array}{l}\text { Centaurium erythraea Rafn } \\
\text { (Gentianaceae) }\end{array}$ & Центорія (Tsentoriia) & Aerial parts & Tea & $\begin{array}{l}\text { Good for the } \\
\text { heart }\end{array}$ & & 2 \\
\hline \multirow[t]{9}{*}{$\begin{array}{l}\text { Chelidonium majus L. (Papaveraceae) } \\
\text { SB003 } \\
\text { NB154;NB078 }\end{array}$} & \multirow[t]{9}{*}{ Rostopască; чистотіл (Chystotil) } & \multirow[t]{8}{*}{ Aerial parts } & Tea & $\begin{array}{l}\text { Good for the } \\
\text { digestive } \\
\text { system }\end{array}$ & 1 & \\
\hline & & & & $\begin{array}{l}\text { Good for the } \\
\text { liver }\end{array}$ & 2 & \\
\hline & & & & $\begin{array}{l}\text { Good for the } \\
\text { stomach }\end{array}$ & 2 & \\
\hline & & & & Liver diseases & 2 & \\
\hline & & & & $\begin{array}{l}\text { Organism } \\
\text { cleansing }\end{array}$ & 4 & \\
\hline & & & & $\begin{array}{l}\text { Stomach } \\
\text { disinfection }\end{array}$ & $4^{\mathrm{a}}$ & \\
\hline & & & $\begin{array}{l}\text { Locally applied } \\
\text { (infused in } \\
\text { alcohol) }\end{array}$ & Joint pain & & 1 \\
\hline & & & $\begin{array}{l}\text { Tincture with } \\
\text { vinegar }\end{array}$ & Joint pain & & $1^{\mathrm{b}}$ \\
\hline & & Sap & $\begin{array}{l}\text { Locally applied } \\
\text { (fresh) }\end{array}$ & Blisters & 1 & \\
\hline $\begin{array}{l}\text { Chenopodium album L. } \\
\text { (Amaranthaceae) } \\
\text { NB139 }\end{array}$ & Натина§, лебеда (Natyna§;lebeda) & Aerial parts & Any Preparation & Healthy & & 1 \\
\hline \multirow{3}{*}{$\begin{array}{l}\text { Cichorium intybus L. (Asteracaeae) } \\
\text { SB046 }\end{array}$} & \multirow{3}{*}{$\begin{array}{l}\text { петрові батоги; петрів батіг } \\
\text { (Petrovi batohy; petriv batih) }\end{array}$} & \multirow[t]{3}{*}{ Aerial parts } & \multirow[t]{3}{*}{ Tea } & Diarrhoea & & 1 \\
\hline & & & & $\begin{array}{l}\text { Good for the } \\
\text { digestive } \\
\text { system }\end{array}$ & & 1 \\
\hline & & & & $\begin{array}{l}\text { Good for the } \\
\text { liver }\end{array}$ & & 1 \\
\hline Coriandrum sativum L. (Apiaceae) & $\begin{array}{l}\text { коляндра; колєндра } \\
\text { (Koliandra; koliendra) }\end{array}$ & Seeds & Tea & Fever & & 7 \\
\hline $\begin{array}{l}\text { Corylus avellana L. (Betulaceae) } \\
\text { SB089 }\end{array}$ & Alune & Leaves & Tea & Prostatitis & 2 & \\
\hline \multirow{7}{*}{$\begin{array}{l}\text { Crataegus spp. including Crataegus } \\
\text { monogyna Jacq. } \\
\text { (Rosaceae) } \\
\text { SB064 } \\
\text { NB066, } \\
\text { NB234 }\end{array}$} & \multirow[t]{7}{*}{$\begin{array}{l}\text { Păducel; бояришнік; глід } \\
\text { (Boiaryshnik^; hlid) }\end{array}$} & \multirow[t]{3}{*}{ Flowers } & $\begin{array}{l}\text { Tincture with } \\
\text { alcohol }\end{array}$ & $\begin{array}{l}\text { Good for the } \\
\text { heart }\end{array}$ & & 3 \\
\hline & & & \multirow{2}{*}{$\begin{array}{l}\text { Infused in } \\
\text { moonshine/ } \\
\text { alcohol }\end{array}$} & $\begin{array}{l}\text { Good for the } \\
\text { heart }\end{array}$ & & 2 \\
\hline & & & & $\begin{array}{l}\text { Good for } \\
\text { blood vessels }\end{array}$ & & 1 \\
\hline & & \multirow[t]{4}{*}{ Fruits } & \multirow[t]{4}{*}{ Tea } & Blood pressure & 1 & 2 \\
\hline & & & & $\begin{array}{l}\text { Good for } \\
\text { cholesterol }\end{array}$ & 1 & \\
\hline & & & & $\begin{array}{l}\text { Good for the } \\
\text { heart }\end{array}$ & 1 & 2 \\
\hline & & & & $\begin{array}{l}\text { Good for } \\
\text { blood vessels }\end{array}$ & & 1 \\
\hline
\end{tabular}


Table 2 Recorded medicinal taxa in Northern and Southern Bukovina. DUR Detailed Use Reports; RO Romanian Hutsuls; UA Ukrainian Hutsuls (Continued)

\begin{tabular}{|c|c|c|c|c|c|c|}
\hline \multirow{2}{*}{$\begin{array}{l}\text { Latin name, family and voucher } \\
\text { specimens }\end{array}$} & \multirow[t]{2}{*}{ Local names } & \multirow[t]{2}{*}{ Used part(s) } & \multirow[t]{2}{*}{ Preparation } & \multirow[t]{2}{*}{ Medicinal Use } & \multicolumn{2}{|c|}{ DUR } \\
\hline & & & & & $\mathrm{RO}$ & $U A$ \\
\hline & & & & Calming & & 1 \\
\hline & & & & Soporific & & 1 \\
\hline & & & Dried & $\begin{array}{l}\text { Good for the } \\
\text { heart }\end{array}$ & & 1 \\
\hline & & & $\begin{array}{l}\text { Tincture with } \\
\text { alcohol }\end{array}$ & $\begin{array}{l}\text { Good for the } \\
\text { heart }\end{array}$ & & 3 \\
\hline \multirow{2}{*}{$\begin{array}{l}\text { Cyanus segetum Hill. } \\
\text { (Asteraceae) }\end{array}$} & \multirow[t]{2}{*}{ Centaurea; васильки (Vasylky) } & Flowers & Tea & Panacea & & 1 \\
\hline & & Aerial parts & Tea & $\begin{array}{l}\text { Good for the } \\
\text { liver }\end{array}$ & 2 & \\
\hline $\begin{array}{l}\text { Daucus carota L. } \\
\text { (Apiaceae) } \\
\text { SB027 }\end{array}$ & Morcov & Roots & Raw & Improve vision & 1 & \\
\hline \multirow{2}{*}{$\begin{array}{l}\text { Dryopteris filix-mas (L.) Schott } \\
\text { (Dryopteridaceae) } \\
\text { NB193 }\end{array}$} & \multirow{2}{*}{$\begin{array}{l}\text { Лісова папороть; солодка } \\
\text { папороть (Lisova paporot; } \\
\text { solodka paporot) }\end{array}$} & Aerial parts & Boiled & $\begin{array}{l}\text { Good for the } \\
\text { heart }\end{array}$ & & 3 \\
\hline & & & Tea & $\begin{array}{l}\text { Good for the } \\
\text { heart }\end{array}$ & & 3 \\
\hline \multirow{4}{*}{$\begin{array}{l}\text { Elaeagnus rhamnoides (L.) A. Nelson } \\
\text { (Elaeagnaceae) }\end{array}$} & \multirow{4}{*}{$\begin{array}{l}\text { Обліпиха } \\
\text { (Oblipykha) }\end{array}$} & Fruits & Oil & Burns & & 1 \\
\hline & & & & Wounds & & 1 \\
\hline & & & Raw with sugar & Healthy & & 1 \\
\hline & & & $\begin{array}{l}\text { Boiled with } \\
\text { sheep fat }\end{array}$ & $\begin{array}{l}\text { Women's } \\
\text { problems }\end{array}$ & & 1 \\
\hline \multirow{3}{*}{$\begin{array}{l}\text { Epilobium angustifolium L. (Onagraceae) } \\
\text { NB057 }\end{array}$} & \multirow{3}{*}{$\begin{array}{l}\text { іван чай,демник§,димник§ } \\
\text { (ivan chai,demnyk§, dymnyk§) }\end{array}$} & Flowers & Tea & Healthy & & 2 \\
\hline & & & & $\begin{array}{l}\text { Good for the } \\
\text { intestines }\end{array}$ & & $1^{a}$ \\
\hline & & & & Healthy & & $1^{a}$ \\
\hline \multirow{6}{*}{$\begin{array}{l}\text { Equisetum arvense L. (Equisetaceae) } \\
\text { SB020 } \\
\text { NB113;NB114 }\end{array}$} & \multirow[t]{6}{*}{$\begin{array}{l}\text { Coada calului; падиволос (хвощ) } \\
\text { (padyvolos (khvoshch)) }\end{array}$} & Aerial parts & Tea & $\begin{array}{l}\text { Good for the } \\
\text { abdomen }\end{array}$ & 1 & \\
\hline & & & & Liver diseases & & 1 \\
\hline & & & & $\begin{array}{l}\text { Good for the } \\
\text { kidneys }\end{array}$ & 1 & \\
\hline & & & & $\begin{array}{l}\text { Good for the } \\
\text { urinary tract }\end{array}$ & 4 & \\
\hline & & & & $\begin{array}{l}\text { Good for the } \\
\text { lungs }\end{array}$ & 2 & \\
\hline & & Flowers & Infusion at $70^{\circ} \mathrm{C}$ & Headache & 1 & \\
\hline \multirow{10}{*}{$\begin{array}{l}\text { Fragaria vesca L. } \\
\text { (Rosaceae) } \\
\text { SB094 } \\
\text { NB004; NB015; NB071; NB240 }\end{array}$} & \multirow{10}{*}{$\begin{array}{l}\text { Fragi; ягоди,лісова ягода; } \\
\text { ягоди лісові; суниці лісові } \\
\text { наз земляніка } \\
\text { (yahody ; lisova yahoda; } \\
\text { yahody lisovi; sunytsi lisovi } \\
\text { naz zemlianika) }\end{array}$} & Aerial parts & Tea & $\begin{array}{l}\text { Good for the } \\
\text { heart }\end{array}$ & 4 & \\
\hline & & & & Healthy & & 1 \\
\hline & & Flowers & Dried & Blood pressure & & 2 \\
\hline & & & Tea & $\begin{array}{l}\text { Good for the } \\
\text { kidneys }\end{array}$ & & 1 \\
\hline & & & Dried & Vitamins & & 3 \\
\hline & & & Dried & Diarrhoea & & 2 \\
\hline & & Fruits & Raw & 100 diseases & & 2 \\
\hline & & & & Fever & & 1 \\
\hline & & & & Healthy & & 1 \\
\hline & & & & $\begin{array}{l}\text { Good for the } \\
\text { skin }\end{array}$ & & 2 \\
\hline
\end{tabular}


Table 2 Recorded medicinal taxa in Northern and Southern Bukovina. DUR Detailed Use Reports; RO Romanian Hutsuls; UA Ukrainian Hutsuls (Continued)

\begin{tabular}{|c|c|c|c|c|c|c|}
\hline \multirow{2}{*}{$\begin{array}{l}\text { Latin name, family and voucher } \\
\text { specimens }\end{array}$} & \multirow[t]{2}{*}{ Local names } & \multirow[t]{2}{*}{ Used part(s) } & \multirow[t]{2}{*}{ Preparation } & \multirow[t]{2}{*}{ Medicinal Use } & \multicolumn{2}{|c|}{ DUR } \\
\hline & & & & & $\mathrm{RO}$ & $\overline{U A}$ \\
\hline Frangula alnus Mill. (Rhamnaceae) & Крушина (Krushyna) & Bark & Boiled & Jaundice & & 1 \\
\hline \multirow{2}{*}{$\begin{array}{l}\text { Galium verum L. } \\
\text { (Rubiaceae) } \\
\text { SB093 }\end{array}$} & \multirow[t]{2}{*}{ Sânziana } & \multirow[t]{2}{*}{ Aerial parts } & Locally applied & $\begin{array}{l}\text { Women's } \\
\text { problems }\end{array}$ & $1^{\mathrm{a}}$ & \\
\hline & & & Tea & $\begin{array}{l}\text { Women's } \\
\text { problems }\end{array}$ & $1^{\mathrm{a}}$ & \\
\hline \multirow[t]{2}{*}{ Gentiana lutea L. (Gentianaceae) } & \multirow[t]{2}{*}{ Gingiura; Джинджора (Dzhyndzhora) } & \multirow[t]{2}{*}{ Roots } & \multirow[t]{2}{*}{$\begin{array}{l}\text { Infused in } \\
\text { alcohol }\end{array}$} & $\begin{array}{l}\text { Good for the } \\
\text { liver }\end{array}$ & & 1 \\
\hline & & & & $\begin{array}{l}\text { Good for the } \\
\text { stomach }\end{array}$ & 1 & \\
\hline Ginkgo biloba L. (Ginkgoaceae) & $\begin{array}{l}\text { Гінго білоба } \\
\text { (Hinho biloba) }\end{array}$ & Leaves & $\begin{array}{l}\text { Infused in } \\
\text { moonshine }\end{array}$ & $\begin{array}{l}\text { Blood } \\
\text { cleansing }\end{array}$ & & $1^{\mathrm{a}}$ \\
\hline $\begin{array}{l}\text { Helianthus annuus L. } \\
\text { (Asteraceae) }\end{array}$ & Соняшник (Soniashnyk) & Fruits & Oil & Constipation & & 1 \\
\hline \multirow[t]{2}{*}{$\begin{array}{l}\text { Helichrysum arenarium (L.) Moench } \\
\text { (Asteraceae) } \\
\text { NB258 }\end{array}$} & \multirow[t]{2}{*}{$\begin{array}{l}\text { Цмин пісковий } \\
\text { (Tsmyn piskovyi) }\end{array}$} & \multirow[t]{2}{*}{ Aerial parts } & \multirow[t]{2}{*}{ Tea (dried) } & $\begin{array}{l}\text { Good for the } \\
\text { digestive } \\
\text { system }\end{array}$ & & 1 \\
\hline & & & & $\begin{array}{l}\text { Stomach } \\
\text { diseases }\end{array}$ & & 1 \\
\hline \multirow{18}{*}{$\begin{array}{l}\text { Hypericum spp. including Hypericum } \\
\text { perforatum L. and, Hypericum } \\
\text { tetrapterum Fr (Hypericaceae) } \\
\text { SB068 } \\
\text { NB080NB101; NB108; NB116 }\end{array}$} & \multirow{18}{*}{$\begin{array}{l}\text { Pojărniță; Sunătoarea; звіробой; } \\
\text { звіробій } \\
\text { (Zviroboi; zvirobii) }\end{array}$} & \multirow[t]{18}{*}{ Aerial parts } & \multirow[t]{13}{*}{ Tea } & Blood pressure & & 2 \\
\hline & & & & $\begin{array}{l}\text { Blood } \\
\text { cleansing }\end{array}$ & & $1^{\mathrm{b}}$ \\
\hline & & & & Diarrhoea & & 3 \\
\hline & & & & $\begin{array}{l}\text { Good for the } \\
\text { liver }\end{array}$ & 7 & \\
\hline & & & & $\begin{array}{l}\text { Good for the } \\
\text { stomach }\end{array}$ & 8 & 3 \\
\hline & & & & $\begin{array}{l}\text { Good for the } \\
\text { gallbladder }\end{array}$ & $1^{\mathrm{a}}$ & \\
\hline & & & & 100 diseases & & 1 \\
\hline & & & & Disinfectant & 1 & \\
\hline & & & & Healthy & 1 & 4 \\
\hline & & & & Panacea & & 1 \\
\hline & & & & $\begin{array}{l}\text { Women's } \\
\text { problems }\end{array}$ & & 2 \\
\hline & & & & Calming & 1 & \\
\hline & & & & $\begin{array}{l}\text { Good for the } \\
\text { eyes }\end{array}$ & 2 & \\
\hline & & & Drink & Evil eye & & $1^{\mathrm{b}}$ \\
\hline & & & \multirow{2}{*}{$\begin{array}{l}\text { Locally applied } \\
\text { (infused in oil) }\end{array}$} & Burns & 4 & 1 \\
\hline & & & & Wounds & 4 & \\
\hline & & & \multirow{2}{*}{$\begin{array}{l}\text { Locally applied } \\
\text { (in spirits with } \\
\text { oil) }\end{array}$} & $\begin{array}{l}\text { Good for the } \\
\text { liver }\end{array}$ & 2 & \\
\hline & & & & $\begin{array}{l}\text { Good for the } \\
\text { stomach }\end{array}$ & 2 & \\
\hline \multirow{3}{*}{$\begin{array}{l}\text { Juglans regia L. (Juglandaceae) } \\
\text { SB051 } \\
\text { NB210 }\end{array}$} & \multirow{3}{*}{$\begin{array}{l}\text { Nuc; ropix } \\
\text { (Horikh) }\end{array}$} & Flowers & Tea & Blood pressure & & 1 \\
\hline & & Fruits & Dried & Healthy & & 3 \\
\hline & & & & $\begin{array}{l}\text { To increase } \\
\text { milk } \\
\text { production in }\end{array}$ & & 2 \\
\hline
\end{tabular}


Table 2 Recorded medicinal taxa in Northern and Southern Bukovina. DUR Detailed Use Reports; RO Romanian Hutsuls; UA Ukrainian Hutsuls (Continued)

\begin{tabular}{|c|c|c|c|c|c|c|}
\hline \multirow{2}{*}{$\begin{array}{l}\text { Latin name, family and voucher } \\
\text { specimens }\end{array}$} & \multirow[t]{2}{*}{ Local names } & \multirow[t]{2}{*}{ Used part(s) } & \multirow[t]{2}{*}{ Preparation } & \multirow[t]{2}{*}{ Medicinal Use } & \multicolumn{2}{|c|}{ DUR } \\
\hline & & & & & $\mathrm{RO}$ & $U A$ \\
\hline & & & & women & & \\
\hline & & & Raw & $\begin{array}{l}\text { To increase } \\
\text { milk } \\
\text { production in } \\
\text { women }\end{array}$ & & 3 \\
\hline & & & & "Jod" & & 1 \\
\hline & & $\begin{array}{l}\text { Inner woody } \\
\text { part of the } \\
\text { fruit }\end{array}$ & $\begin{array}{l}\text { Infused in } \\
\text { alcohol }\end{array}$ & $\begin{array}{l}\text { Good for the } \\
\text { thyroid }\end{array}$ & & 1 \\
\hline & & Leaves & Tea & $\begin{array}{l}\text { Good for the } \\
\text { heart }\end{array}$ & & 1 \\
\hline & & & & Hair care & 1 & $1^{b}$ \\
\hline & & Unripe fruits & $\begin{array}{l}\text { Infused in } \\
\text { alcohol }\end{array}$ & $\begin{array}{l}\text { Good for the } \\
\text { thyroid }\end{array}$ & & 1 \\
\hline \multirow[t]{2}{*}{$\begin{array}{l}\text { Juniperus communis L. (Cupressaceae) } \\
\text { SB086 }\end{array}$} & \multirow[t]{2}{*}{$\begin{array}{l}\text { lenupăr; жуніпера } \\
\text { (zhunipera) }\end{array}$} & Fruits & Tea & $\begin{array}{l}\text { Good for the } \\
\text { liver }\end{array}$ & 1 & \\
\hline & & Leaves & Tea & $\begin{array}{l}\text { Good for the } \\
\text { kidneys }\end{array}$ & & 2 \\
\hline \multirow{3}{*}{$\begin{array}{l}\text { Lamium album L. } \\
\text { (Lamiaceae) } \\
\text { NB216 }\end{array}$} & \multirow{3}{*}{$\begin{array}{l}\text { Кропива собача; біла кропива } \\
\text { нежалка; глуха кропива } \\
\text { (Kropyva sobacha; bila } \\
\text { kropyva nezhalka; hlukha kropyva) }\end{array}$} & Aerial parts & Tea & Blood pressure & & 1 \\
\hline & & & & $\begin{array}{l}\text { Good for the } \\
\text { heart }\end{array}$ & & 3 \\
\hline & & & & Nerves & & 1 \\
\hline \multirow{8}{*}{$\begin{array}{l}\text { Leonurus cardiaca L. (Lamiaceae) } \\
\text { SB013 }\end{array}$} & \multirow{8}{*}{$\begin{array}{l}\text { Talpa gâștei; пустирник } \\
\text { (Pustyrnyk) }\end{array}$} & \multirow[t]{6}{*}{ Aerial parts } & \multirow[t]{6}{*}{ Tea } & Blood pressure & & 1 \\
\hline & & & & $\begin{array}{l}\text { Good for the } \\
\text { heart }\end{array}$ & 6 & 1 \\
\hline & & & & Healthy & 2 & \\
\hline & & & & Pain & 1 & \\
\hline & & & & Nerves & 2 & \\
\hline & & & & Rhinitis & 4 & \\
\hline & & \multirow[t]{2}{*}{ Leaves } & \multirow{2}{*}{$\begin{array}{l}\text { Locally applied } \\
\text { (fresh with pork } \\
\text { fat) }\end{array}$} & Cuts & 2 & \\
\hline & & & & Warts & 2 & \\
\hline \multirow{2}{*}{$\begin{array}{l}\text { Levisticum officinale W.D.J.Koch } \\
\text { (Apiaceae) }\end{array}$} & \multirow[t]{2}{*}{ Любисток (Liubystok) } & \multirow[t]{2}{*}{ Aerial parts } & \multirow[t]{2}{*}{ Tea } & Alcoholism & & 1 \\
\hline & & & & Hair care & & 3 \\
\hline \multirow{8}{*}{$\begin{array}{l}\text { Lilium candidum L. (Amaryllidaceae) } \\
\text { SB049 }\end{array}$} & \multirow{8}{*}{$\begin{array}{l}\text { Crin alb; Narcise; лілія біла; } \\
\text { лилия } \\
\text { (Liliia bila; lylyia) }\end{array}$} & \multirow[t]{8}{*}{ Flowers } & \multirow{7}{*}{$\begin{array}{l}\text { Locally applied } \\
\text { (infused in } \\
\text { alcohol) } \\
\text { Locally applied } \\
\text { (infused in } \\
\text { alcohol) } \\
\text { Locally applied } \\
\text { (in spirits, } \\
\text { medicinal) }\end{array}$} & Good for veins & 1 & \\
\hline & & & & Bee stings & & 1 \\
\hline & & & & Burns & & 1 \\
\hline & & & & Warts & & 5 \\
\hline & & & & Wounds & & 6 \\
\hline & & & & Joint pain & 1 & \\
\hline & & & & Tired feet & 1 & \\
\hline & & & $\begin{array}{l}\text { Drink (infused } \\
\text { in alcohol) }\end{array}$ & Healthy & & 1 \\
\hline \multirow[t]{2}{*}{ Linum usitatissimum L. (Linaceae) } & \multirow[t]{2}{*}{$\begin{array}{l}\text { Lin; лен; льон } \\
\text { (Len; lon) }\end{array}$} & \multirow[t]{2}{*}{ Seeds } & \multirow[t]{2}{*}{ Tea } & $\begin{array}{l}\text { Good for the } \\
\text { stomach }\end{array}$ & 1 & $2^{b}$ \\
\hline & & & & $\begin{array}{l}\text { To increase } \\
\text { milk } \\
\text { production in } \\
\text { women }\end{array}$ & & 3 \\
\hline
\end{tabular}


Table 2 Recorded medicinal taxa in Northern and Southern Bukovina. DUR Detailed Use Reports; RO Romanian Hutsuls; UA Ukrainian Hutsuls (Continued)

\begin{tabular}{|c|c|c|c|c|c|c|}
\hline \multirow{2}{*}{$\begin{array}{l}\text { Latin name, family and voucher } \\
\text { specimens }\end{array}$} & \multirow[t]{2}{*}{ Local names } & \multirow[t]{2}{*}{ Used part(s) } & \multirow[t]{2}{*}{ Preparation } & \multirow[t]{2}{*}{ Medicinal Use } & \multicolumn{2}{|c|}{ DUR } \\
\hline & & & & & $\mathrm{RO}$ & UA \\
\hline \multirow[t]{3}{*}{ Lonicera caprifolium L. (Caprifoliaceae) } & \multirow[t]{3}{*}{ Floarea maicii domnului } & \multirow[t]{3}{*}{ Aerial parts } & \multirow{3}{*}{$\begin{array}{l}\text { Locally applied } \\
\text { (dried tea) }\end{array}$} & Wounds & 1 & \\
\hline & & & & $\begin{array}{l}\text { Women's } \\
\text { problems }\end{array}$ & 1 & \\
\hline & & & & Measles & 1 & \\
\hline $\begin{array}{l}\text { Lycopodium clavatum } \\
\text { L. (Lycopodiaceae) } \\
\text { NB231 }\end{array}$ & $\begin{array}{l}\text { Плаун } \\
\text { (Plaun) }\end{array}$ & Aerial parts & Dried & Wounds & & 2 \\
\hline \multirow[t]{2}{*}{$\begin{array}{l}\text { Maclura pomifera (Raf.) C.K.Schneid. } \\
\text { (Moraceae) }\end{array}$} & \multirow[t]{2}{*}{$\begin{array}{l}\text { Адамове яблуко матлюрка } \\
\text { (Adamove yabluko matliurka) }\end{array}$} & \multirow[t]{2}{*}{ Fruits } & \multirow{2}{*}{$\begin{array}{l}\text { Locally applied } \\
\text { (infused in } \\
\text { alcohol) }\end{array}$} & $\begin{array}{l}\text { Women's } \\
\text { problems }\end{array}$ & & 2 \\
\hline & & & & Joint pain & & 2 \\
\hline $\begin{array}{l}\text { Malus domestica Borkh. } \\
\text { (Rosaceae) } \\
\text { NB242 }\end{array}$ & $\begin{array}{l}\text { Яблука } \\
\text { (Yabluka) }\end{array}$ & Fruits & $\begin{array}{l}\text { Boiled with } \\
\text { onion }\end{array}$ & Cough & & 3 \\
\hline \multirow{22}{*}{$\begin{array}{l}\text { Matricaria chamomilla L. (Asteraceae) } \\
\text { SB019; SB022 } \\
\text { NB164;NB171 }\end{array}$} & \multirow{22}{*}{$\begin{array}{l}\text { Mușețel; Romaniță; ромашка; румянець } \\
\text { (Romashka; rumianets) }\end{array}$} & \multirow[t]{4}{*}{ Aerial parts } & \multirow[t]{4}{*}{ Tea (dried) } & Red skin & & 1 \\
\hline & & & & $\begin{array}{l}\text { Good for the } \\
\text { digestive } \\
\text { system }\end{array}$ & & 1 \\
\hline & & & & $\begin{array}{l}\text { Inflammation } \\
\text { processes }\end{array}$ & & 1 \\
\hline & & & & $\begin{array}{l}\text { Good for the } \\
\text { throat }\end{array}$ & & 1 \\
\hline & & \multirow[t]{18}{*}{ Flowers } & Poultice (dried) & Evil eye & 1 & \\
\hline & & & \multirow[t]{5}{*}{ Compress } & Skin infections & 1 & \\
\hline & & & & Warts & 1 & \\
\hline & & & & Eye cleaning & 2 & \\
\hline & & & & Eye problems & 1 & 3 \\
\hline & & & & $\begin{array}{l}\text { Good for the } \\
\text { eyes }\end{array}$ & 1 & 2 \\
\hline & & & \multirow[t]{10}{*}{ Tea } & Diarrhoea & 1 & \\
\hline & & & & $\begin{array}{l}\text { Good for the } \\
\text { stomach }\end{array}$ & 2 & 3 \\
\hline & & & & Disinfectant & 1 & \\
\hline & & & & Healthy & 1 & 3 \\
\hline & & & & Panacea & 1 & 5 \\
\hline & & & & $\begin{array}{l}\text { Good for the } \\
\text { urinary tract }\end{array}$ & 2 & \\
\hline & & & & Headache & 2 & \\
\hline & & & & $\begin{array}{l}\text { Wound } \\
\text { cleansing }\end{array}$ & & $1^{b}$ \\
\hline & & & & Gum problems & 1 & \\
\hline & & & & Colds & 2 & $1^{c}$ \\
\hline & & & \multirow{2}{*}{$\begin{array}{l}\text { Tea with } O . \\
\text { Vulgare }\end{array}$} & Gum problems & 1 & \\
\hline & & & & Disinfectant & 1 & \\
\hline \multirow[t]{4}{*}{ Melissa officinalis L. (Lamiaceae) } & \multirow[t]{4}{*}{ Меліса (Melisa) } & \multirow[t]{4}{*}{ Leaves } & \multirow[t]{4}{*}{ Tea (dried) } & Healthy & & 2 \\
\hline & & & & Pain & & 2 \\
\hline & & & & Calming & & 3 \\
\hline & & & & Headache & & 1 \\
\hline
\end{tabular}


Table 2 Recorded medicinal taxa in Northern and Southern Bukovina. DUR Detailed Use Reports; RO Romanian Hutsuls; UA Ukrainian Hutsuls (Continued)

\begin{tabular}{|c|c|c|c|c|c|c|}
\hline \multirow{2}{*}{$\begin{array}{l}\text { Latin name, family and voucher } \\
\text { specimens }\end{array}$} & \multirow[t]{2}{*}{ Local names } & \multirow[t]{2}{*}{ Used part(s) } & \multirow[t]{2}{*}{ Preparation } & \multirow[t]{2}{*}{ Medicinal Use } & \multicolumn{2}{|c|}{ DUR } \\
\hline & & & & & $\overline{\mathrm{RO}}$ & $\overline{U A}$ \\
\hline & & & & Soporific & & 1 \\
\hline & & & & Stress & & 1 \\
\hline \multirow{14}{*}{$\begin{array}{l}\text { Mentha spp. } \\
\text { (Lamiaceae) } \\
\text { SB014; SB016; SB034; SB096 } \\
\text { NB079;NB080;NB097 }\end{array}$} & \multirow{14}{*}{$\begin{array}{l}\text { Mentă; мята; мятка; мята гладка; мята } \\
\text { кучерява; мятка кінська; мятка перчева } \\
\text { (Miata; miatka; miata hladka; miata kucheriava; } \\
\text { miatka kinska; miatka percheva) }\end{array}$} & \multirow[t]{14}{*}{ Aerial parts } & \multirow[t]{13}{*}{ Tea } & $\begin{array}{l}\text { Good for the } \\
\text { heart }\end{array}$ & & 6 \\
\hline & & & & Heart disease & & 1 \\
\hline & & & & Diarrhoea & 1 & \\
\hline & & & & $\begin{array}{l}\text { Good for the } \\
\text { stomach }\end{array}$ & & 1 \\
\hline & & & & $\begin{array}{l}\text { Stomach } \\
\text { problems }\end{array}$ & & 2 \\
\hline & & & & Vomiting & & 1 \\
\hline & & & & Healthy & 1 & \\
\hline & & & & Pain & & 1 \\
\hline & & & & Diuretic & 1 & \\
\hline & & & & $\begin{array}{l}\text { Good for the } \\
\text { urinary tract }\end{array}$ & 2 & \\
\hline & & & & Calming & 1 & 1 \\
\hline & & & & Headache & & 2 \\
\hline & & & & Stress & & 1 \\
\hline & & & $\begin{array}{l}\text { Locally applied } \\
\text { (infused in } \\
\text { alcohol) }\end{array}$ & Joint pain & & 1 \\
\hline \multirow{18}{*}{$\begin{array}{l}\text { Origanum vulgare L. (Lamiaceae) } \\
\text { SB036 } \\
\text { NB033; NB055; NB021 }\end{array}$} & \multirow{18}{*}{$\begin{array}{l}\text { Șovârv; Șovârf; Materanca bila; } \\
\text { материнкa } \\
\text { (Materynka) }\end{array}$} & \multirow[t]{18}{*}{ Aerial parts } & \multirow[t]{18}{*}{ Tea } & Blood pressure & 4 & \\
\hline & & & & $\begin{array}{l}\text { Blood } \\
\text { regeneration }\end{array}$ & & 1 \\
\hline & & & & $\begin{array}{l}\text { Good for the } \\
\text { heart }\end{array}$ & 4 & 2 \\
\hline & & & & Red skin & & 2 \\
\hline & & & & $\begin{array}{l}\text { Abdominal } \\
\text { pain }\end{array}$ & 2 & \\
\hline & & & & Diarrhoea & & 1 \\
\hline & & & & $\begin{array}{l}\text { Good for the } \\
\text { liver }\end{array}$ & 3 & \\
\hline & & & & $\begin{array}{l}\text { Good for the } \\
\text { stomach }\end{array}$ & 7 & 2 \\
\hline & & & & 100 diseases & & 2 \\
\hline & & & & Disinfectant & 1 & \\
\hline & & & & Fever & 1 & \\
\hline & & & & Healthy & & 2 \\
\hline & & & & $\begin{array}{l}\text { Inflammation } \\
\text { processes }\end{array}$ & & 2 \\
\hline & & & & Panacea & & 1 \\
\hline & & & & Leptospirosis & 1 & \\
\hline & & & & Septicaemia & 1 & \\
\hline & & & & $\begin{array}{l}\text { Good for the } \\
\text { kidneys }\end{array}$ & 1 & \\
\hline & & & & Soporific & & $1^{\mathrm{b}}$ \\
\hline
\end{tabular}


Table 2 Recorded medicinal taxa in Northern and Southern Bukovina. DUR Detailed Use Reports; RO Romanian Hutsuls; UA Ukrainian Hutsuls (Continued)

\begin{tabular}{|c|c|c|c|c|c|c|}
\hline \multirow{2}{*}{$\begin{array}{l}\text { Latin name, family and voucher } \\
\text { specimens }\end{array}$} & \multirow[t]{2}{*}{ Local names } & \multirow[t]{2}{*}{ Used part(s) } & \multirow[t]{2}{*}{ Preparation } & \multirow[t]{2}{*}{ Medicinal Use } & \multicolumn{2}{|c|}{ DUR } \\
\hline & & & & & $\mathrm{RO}$ & UA \\
\hline & & & & $\begin{array}{l}\text { Good for the } \\
\text { lungs }\end{array}$ & & 1 \\
\hline & & $\begin{array}{l}\text { Aerial parts } \\
\text { (flowers) }\end{array}$ & Tea & $\begin{array}{l}\text { Women's } \\
\text { problems }\end{array}$ & & 8 \\
\hline Panax ginseng C.A. Mey. (Araliaceae) & Женшень (Zhenshen) & Roots & $\begin{array}{l}\text { Infused in } \\
\text { alcohol }\end{array}$ & Blood pressure & & 1 \\
\hline $\begin{array}{l}\text { Papaver somniferum L. } \\
\text { (Papaveraceae) }\end{array}$ & Mak (Mak) & Aerial parts & Tea & Soporific & & $2^{b}$ \\
\hline $\begin{array}{l}\text { Petroselinum crispum (Mill.) Fuss } \\
\text { (Apiaceae) } \\
\text { NB220 }\end{array}$ & Петрушка (Petrushka) & Leaves & Raw & Vitamins & & 2 \\
\hline Phaseolus vulgaris L. (Leguminosae) & Фасоля (Fasolia) & Pod & Tea & Diabetes & & 2 \\
\hline \multirow{21}{*}{$\begin{array}{l}\text { Picea abies (L.) H. Karst. possibly } \\
\text { including Abies alba Mill. (Pinaceae) } \\
\text { SB008 } \\
\text { NB043 }\end{array}$} & \multirow{21}{*}{$\begin{array}{l}\text { Brad; смерека; хвоя } \\
\text { (Smereka, khvoya) }\end{array}$} & \multirow[t]{3}{*}{ Flowers } & \multirow[t]{3}{*}{ Syrup } & Bronchitis & & 2 \\
\hline & & & & Cough & & 4 \\
\hline & & & & $\begin{array}{l}\text { Good for } \\
\text { breathing }\end{array}$ & & 2 \\
\hline & & \multirow[t]{3}{*}{ Needles } & Syrup & Cough & & 2 \\
\hline & & & \multirow[t]{2}{*}{ Tea } & Cough & & 2 \\
\hline & & & & $\begin{array}{l}\text { Good for the } \\
\text { throat }\end{array}$ & & 2 \\
\hline & & Resin & Locally applied & Joint pain & & 2 \\
\hline & & \multirow{11}{*}{$\begin{array}{l}\text { Sprouts } \\
\text { (young) }\end{array}$} & \multirow[t]{9}{*}{ Syrup } & Fever & 1 & \\
\hline & & & & Panacea & 2 & \\
\hline & & & & Colds & 4 & \\
\hline & & & & Cough & 8 & \\
\hline & & & & $\begin{array}{l}\text { Good for the } \\
\text { lungs }\end{array}$ & 2 & \\
\hline & & & & $\begin{array}{l}\text { Good for the } \\
\text { respiratory } \\
\text { system }\end{array}$ & 2 & \\
\hline & & & & $\begin{array}{l}\text { Good for the } \\
\text { throat }\end{array}$ & 1 & \\
\hline & & & & Bronchitis & & 2 \\
\hline & & & & Sore throat & 1 & \\
\hline & & & \multirow[t]{2}{*}{ Essence (fresh) } & Panacea & 2 & \\
\hline & & & & Colds & 2 & \\
\hline & & \multirow[t]{3}{*}{ Young cones } & \multirow[t]{2}{*}{ Syrup } & Bronchitis & & 2 \\
\hline & & & & Cough & & 4 \\
\hline & & & With sugar & Pneumonia & & 1 \\
\hline $\begin{array}{l}\text { Pinus sylvestris L. } \\
\text { (Pinaceae) }\end{array}$ & Pin & Young sprouts & Syrup (fresh) & Cough & 1 & \\
\hline $\begin{array}{l}\text { Plantago lanceolata L. } \\
\text { (Plantaginaceae) } \\
\text { SB037 }\end{array}$ & $\begin{array}{l}\text { Pătlagină îngusta; подорожник } \\
\text { ланцеолистий } \\
\text { (Podorozhnyk lantseolystyi) }\end{array}$ & Leaves & Tea (fresh) & Cough & 1 & 1 \\
\hline \multirow{3}{*}{$\begin{array}{l}\text { Plantago major L. (Plantaginaceae) } \\
\text { SB066 } \\
\text { NB022;NB047; NB132 }\end{array}$} & \multirow{3}{*}{$\begin{array}{l}\text { Platagine; Platagină; Podorojnic; } \\
\text { подорожник (Podorozhnyk) }\end{array}$} & \multirow[t]{3}{*}{ Leaves } & \multirow{3}{*}{$\begin{array}{l}\text { Locally applied } \\
\text { (fresh) }\end{array}$} & Abscesses & & 2 \\
\hline & & & & Cuts & 1 & 1 \\
\hline & & & & $\begin{array}{l}\text { Good for the } \\
\text { skin }\end{array}$ & 2 & \\
\hline
\end{tabular}


Table 2 Recorded medicinal taxa in Northern and Southern Bukovina. DUR Detailed Use Reports; RO Romanian Hutsuls; UA Ukrainian Hutsuls (Continued)

\begin{tabular}{|c|c|c|c|c|c|c|}
\hline \multirow{2}{*}{$\begin{array}{l}\text { Latin name, family and voucher } \\
\text { specimens }\end{array}$} & \multirow[t]{2}{*}{ Local names } & \multirow[t]{2}{*}{ Used part(s) } & \multirow[t]{2}{*}{ Preparation } & \multirow[t]{2}{*}{ Medicinal Use } & \multicolumn{2}{|c|}{ DUR } \\
\hline & & & & & $\mathrm{RO}$ & UA \\
\hline & & & & Pus & 1 & \\
\hline & & & & Skin infections & 1 & \\
\hline & & & & Skin irritation & 1 & \\
\hline & & & & Disinfectant & & $1^{\mathrm{b}}$ \\
\hline & & & & Sores & 1 & \\
\hline & & & & Warts & 3 & 3 \\
\hline & & & & Wounds & 1 & 15 \\
\hline & & & $\begin{array}{l}\text { Locally applied } \\
\text { (with alcohol) }\end{array}$ & Wounds & & 2 \\
\hline & & & Tea & Cough & & 2 \\
\hline & & Seeds & Tea & $\begin{array}{l}\text { Good for the } \\
\text { kidneys }\end{array}$ & & 2 \\
\hline & & Whole plant & Tea & $\begin{array}{l}\text { Women's } \\
\text { problems }\end{array}$ & & 1 \\
\hline Potentilla anserina L. (Rosaceae) & Coada racului & Aerial parts & Tea & Indigestion & 1 & \\
\hline \multirow[t]{5}{*}{ Potentilla erecta (L.) Raeusch. (Rosaceae) } & \multirow{5}{*}{$\begin{array}{l}\text { Калган; калган; перстач } \\
\text { прямостоячий } \\
\text { (Kalhan; kalhan; perstach } \\
\text { priamostoiachyi) }\end{array}$} & \multirow[t]{5}{*}{ Roots } & Tea & $\begin{array}{l}\text { Reproductive } \\
\text { potency }\end{array}$ & & 1 \\
\hline & & & Tea (dried) & $\begin{array}{l}\text { Reproductive } \\
\text { potency }\end{array}$ & & 1 \\
\hline & & & \multirow{3}{*}{$\begin{array}{l}\text { Infused in } \\
\text { alcohol }\end{array}$} & Good for men & & 1 \\
\hline & & & & Joint pain & & 2 \\
\hline & & & & $\begin{array}{l}\text { Good for the } \\
\text { thyroid }\end{array}$ & & 1 \\
\hline \multirow{4}{*}{$\begin{array}{l}\text { Primula spp. including Primula veris } \mathrm{L} \text {. } \\
\text { and Primula elatior (L.) Hill } \\
\text { (Primulaceae) }\end{array}$} & \multirow{4}{*}{$\begin{array}{l}\text { Ciobațica cucului; Cioboțica } \\
\text { cucului; первоцвіт буковинський } \\
\text { (Pervotsvit bukovynskyi) }\end{array}$} & \multirow[t]{3}{*}{ Aerial parts } & \multirow[t]{3}{*}{ Tea } & $\begin{array}{l}\text { Good for the } \\
\text { heart }\end{array}$ & 1 & \\
\hline & & & & $\begin{array}{l}\text { Good for the } \\
\text { liver }\end{array}$ & 1 & \\
\hline & & & & $\begin{array}{l}\text { Good for the } \\
\text { stomach }\end{array}$ & & 2 \\
\hline & & Flowers & Tea & Cough & & 3 \\
\hline \multirow{2}{*}{$\begin{array}{l}\text { Prunus avium (L.) L. (Rosaceae) } \\
\text { SB059 }\end{array}$} & \multirow[t]{2}{*}{ Cireș } & \multirow[t]{2}{*}{ Stalks } & \multirow[t]{2}{*}{ Tea } & Diuretic & 1 & \\
\hline & & & & $\begin{array}{l}\text { Good for the } \\
\text { kidneys }\end{array}$ & 1 & \\
\hline $\begin{array}{l}\text { Pteridium aquilinum (L.) Kuhn } \\
\text { (Dennstaedtiaceae) } \\
\text { NB074 }\end{array}$ & Папороть орляк (Paporot orliak) & Aerial parts & Bath & $\begin{array}{l}\text { Women's } \\
\text { problems }\end{array}$ & & 1 \\
\hline \multirow[t]{2}{*}{$\begin{array}{l}\text { Pyrus pyraster } \\
\text { (L.) Burgsd. (Rosaceae) }\end{array}$} & \multirow[t]{2}{*}{ Дика груша (Dyka hrusha) } & \multirow[t]{2}{*}{ Fruits } & Tea & $\begin{array}{l}\text { Salt in the } \\
\text { joints }\end{array}$ & & 2 \\
\hline & & & $\begin{array}{l}\text { Infused in } \\
\text { spirits }\end{array}$ & $\begin{array}{l}\text { Salt in the } \\
\text { joints }\end{array}$ & & 2 \\
\hline \multirow{2}{*}{$\begin{array}{l}\text { Quercus spp. including } \\
\text { Quercus robur L. and Quercus rubra L. }\end{array}$} & \multirow[t]{2}{*}{ Дуб (Dub) } & \multirow[t]{2}{*}{ Bark } & Boiled & Gangrene & & 2 \\
\hline & & & Tea & Toothache & & 1 \\
\hline \multirow{2}{*}{$\begin{array}{l}\text { Raphanus sativus L. (Brassicaceae) } \\
\text { NB250 }\end{array}$} & \multirow[t]{2}{*}{ Редька чорна (Redka chorna) } & \multirow[t]{2}{*}{ Roots } & \multirow[t]{2}{*}{ Baked } & Cough & & 2 \\
\hline & & & & $\begin{array}{l}\text { Good for } \\
\text { breathing }\end{array}$ & & 2 \\
\hline Ribes nigrum L. (Grossulariaceae) & Coacăză neagră ; смородина; & Aerial parts & Tea & Cough & & 2 \\
\hline $\begin{array}{l}\mathrm{SBO42} \\
\mathrm{NB} 211\end{array}$ & $\begin{array}{l}\text { чорна смородина (Smorodyna; } \\
\text { chorna smorodyna) }\end{array}$ & Fruits & Juice & Blood pressure & & 1 \\
\hline & & & Jam & Good for & & 1 \\
\hline
\end{tabular}


Table 2 Recorded medicinal taxa in Northern and Southern Bukovina. DUR Detailed Use Reports; RO Romanian Hutsuls; UA Ukrainian Hutsuls (Continued)

\begin{tabular}{|c|c|c|c|c|c|c|}
\hline \multirow{2}{*}{$\begin{array}{l}\text { Latin name, family and voucher } \\
\text { specimens }\end{array}$} & \multirow[t]{2}{*}{ Local names } & \multirow[t]{2}{*}{ Used part(s) } & \multirow[t]{2}{*}{ Preparation } & \multirow[t]{2}{*}{ Medicinal Use } & \multicolumn{2}{|c|}{ DUR } \\
\hline & & & & & $\mathrm{RO}$ & UA \\
\hline & & & & haemoglobin & & \\
\hline & & & Jam & $\begin{array}{l}\text { Good for the } \\
\text { eyes }\end{array}$ & & 1 \\
\hline & & & Raw & Blood pressure & & 3 \\
\hline & & & & $\begin{array}{l}\text { Good for the } \\
\text { intestines }\end{array}$ & 2 & \\
\hline & & & & Vitamins & 2 & \\
\hline \multirow{5}{*}{$\begin{array}{l}\text { Ribes rubrum L. } \\
\text { (Grossulariaceae) } \\
\text { SB042 } \\
\text { NB213 }\end{array}$} & \multirow{5}{*}{ 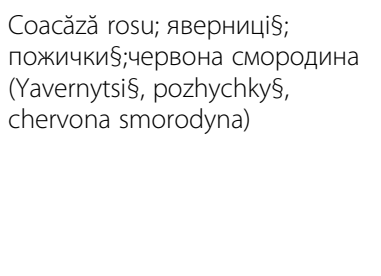 } & \multirow[t]{5}{*}{ Fruits } & Raw & $\begin{array}{l}\text { Good for the } \\
\text { intestines }\end{array}$ & 2 & \\
\hline & & & & Vitamins & 2 & \\
\hline & & & & Kidneys stones & & 2 \\
\hline & & & Tea & Fever & & 1 \\
\hline & & & & Flu & & 1 \\
\hline \multirow{11}{*}{$\begin{array}{l}\text { Rosa canina L. } \\
\text { (Rosaceae) } \\
\text { SB062 } \\
\text { NB018; NB083 }\end{array}$} & \multirow[t]{11}{*}{$\begin{array}{l}\text { Măceșe; Măcess de padure; } \\
\text { шипшина (Shypshyna) }\end{array}$} & \multirow[t]{10}{*}{ Fruits } & Tea & $\begin{array}{l}\text { Good for the } \\
\text { heart }\end{array}$ & 1 & \\
\hline & & & & $\begin{array}{l}\text { Good for the } \\
\text { kidneys }\end{array}$ & & 2 \\
\hline & & & & Healthy & & 2 \\
\hline & & & & $\begin{array}{l}\text { Immune } \\
\text { boosting }\end{array}$ & & 1 \\
\hline & & & & Vitamins & & 1 \\
\hline & & & & $\begin{array}{l}\text { Good for the } \\
\text { kidneys }\end{array}$ & & 1 \\
\hline & & & & $\begin{array}{l}\text { Good for the } \\
\text { urinary tract }\end{array}$ & 2 & \\
\hline & & & & Cough & 4 & \\
\hline & & & & Flu & 4 & \\
\hline & & & Syrup (fresh) & Cold & 1 & \\
\hline & & Roots & Tea & $\begin{array}{l}\text { Good for the } \\
\text { kidneys }\end{array}$ & & 3 \\
\hline \multirow{2}{*}{$\begin{array}{l}\text { Rosa rugosa L. and Rosa centifolia L. } \\
\text { (Rosaceae) } \\
\text { SB023 }\end{array}$} & \multirow[t]{2}{*}{ Trandafir; роза (Roza) } & Flowers & Tea & Calming & & 1 \\
\hline & & Petals & Juice & $\begin{array}{l}\text { Foot-and- } \\
\text { mouth disease }\end{array}$ & 1 & \\
\hline \multirow{6}{*}{$\begin{array}{l}\text { Rubus caesius L. } \\
\text { (Rosaceae) } \\
\text { NB062; NB063 }\end{array}$} & \multirow{6}{*}{$\begin{array}{l}\text { Ожина; чониця; єжевіка; ожина } \\
\text { (Ozhyna; chonytsia; yezhevika; } \\
\text { ozhyna) }\end{array}$} & \multirow[t]{6}{*}{ Fruits } & $\begin{array}{l}\text { Infused in } \\
\text { alcohol }\end{array}$ & 101 diseases & & 2 \\
\hline & & & Boiled & Healthy & & 1 \\
\hline & & & & Cancer & & 1 \\
\hline & & & (with sugar) & Vitamins & & 5 \\
\hline & & & & Colds & & 1 \\
\hline & & & & $\mathrm{Flu}$ & & 1 \\
\hline \multirow{6}{*}{$\begin{array}{l}\text { Rubus idaeus L. } \\
\text { (Rosaceae) } \\
\text { SB071; SB009 } \\
\text { NB081 }\end{array}$} & \multirow{6}{*}{$\begin{array}{l}\text { Zmeură; малина } \\
\text { (malyna) }\end{array}$} & \multirow[t]{6}{*}{ Aerial parts } & Tea & Diabetes & 1 & \\
\hline & & & & Diabetes & & 1 \\
\hline & & & & Fever & 3 & 6 \\
\hline & & & & Healthy & & 3 \\
\hline & & & & $\begin{array}{l}\text { Good for the } \\
\text { kidneys }\end{array}$ & & 1 \\
\hline & & & & Colds & 2 & 3 \\
\hline
\end{tabular}


Table 2 Recorded medicinal taxa in Northern and Southern Bukovina. DUR Detailed Use Reports; RO Romanian Hutsuls; UA Ukrainian Hutsuls (Continued)

\begin{tabular}{|c|c|c|c|c|c|c|}
\hline \multirow{2}{*}{$\begin{array}{l}\text { Latin name, family and voucher } \\
\text { specimens }\end{array}$} & \multirow[t]{2}{*}{ Local names } & \multirow[t]{2}{*}{ Used part(s) } & \multirow[t]{2}{*}{ Preparation } & \multirow[t]{2}{*}{ Medicinal Use } & \multicolumn{2}{|c|}{ DUR } \\
\hline & & & & & $\mathrm{RO}$ & UA \\
\hline & & & & Cough & & 3 \\
\hline & & & & Flu & & 1 \\
\hline & & Fruits & Tea & Blood pressure & & 2 \\
\hline & & & Jam & $\begin{array}{l}\text { Good for } \\
\text { haemoglobin }\end{array}$ & & 1 \\
\hline & & & Dried & Fever & & 3 \\
\hline & & & Juice & Fever & & 6 \\
\hline & & & & Colds & 1 & \\
\hline & & & & Cough & 1 & \\
\hline & & & $\begin{array}{l}\text { Juice With } \\
\text { Lemon }\end{array}$ & Fever & 1 & \\
\hline & & & $\begin{array}{l}\text { Locally applied } \\
\text { syrup without } \\
\text { sugar }\end{array}$ & Fever & 1 & 1 \\
\hline & & & Moonshine & Healthy & & $1^{\mathrm{a}}$ \\
\hline & & & Syrup (without & Fever & 1 & 3 \\
\hline & & & $\begin{array}{l}\text { sugar, with } \\
\text { mashed } \\
\text { potatoes ) }\end{array}$ & Colds & 1 & \\
\hline & & & Raw (with & Panacea & & 1 \\
\hline & & & sugar) & Flu & & 1 \\
\hline & & & & Vitamins & & 5 \\
\hline & & & & Colds & 2 & 1 \\
\hline & & & & 100 diseases & & 2 \\
\hline & & & Syrup (fresh) & Cough & 1 & \\
\hline & & & & Flu & 4 & 1 \\
\hline & & & & Colds & 1 & \\
\hline & & & & Fever & 5 & \\
\hline & & & & $\begin{array}{l}\text { Strengthening } \\
\text { of the } \\
\text { organism }\end{array}$ & 1 & \\
\hline & & & & $\begin{array}{l}\text { Good for the } \\
\text { lungs }\end{array}$ & 1 & \\
\hline \multirow{3}{*}{$\begin{array}{l}\text { Rumex acetosa L. (Polygonaceae) } \\
\text { NB081 }\end{array}$} & \multirow{3}{*}{$\begin{array}{l}\text { Măcriș; квас§; квасок§; щавель } \\
\text { (Kvas§; kvasok§; shchavel) }\end{array}$} & Aerial parts & Any preparation & Vitamins & & 3 \\
\hline & & Roots & Tea & $\begin{array}{l}\text { Good for the } \\
\text { liver }\end{array}$ & 1 & \\
\hline & & & & Colds & 2 & \\
\hline \multirow[t]{2}{*}{$\begin{array}{l}\text { Rumex alpinus L. (Polygonaceae) } \\
\text { SB067 }\end{array}$} & \multirow[t]{2}{*}{ Ștevie } & Leaves & $\begin{array}{l}\text { Locally applied } \\
\text { (fresh) }\end{array}$ & Cuts & 2 & \\
\hline & & & Tea & Diarrhoea & 1 & \\
\hline $\begin{array}{l}\text { Salix } \times \text { fragilis } \mathrm{L} \text {. } \\
\text { (Salicaceae) }\end{array}$ & Верба ламка (Verba lamka) & Bark & Tea & Fever & & 1 \\
\hline $\begin{array}{l}\text { Salvia pratensis } \mathrm{L} \text {. } \\
\text { (Lamiaceae) } \\
\text { SB028 }\end{array}$ & Salvia & Aerial parts & Tea & Calming & 1 & \\
\hline \multirow{2}{*}{$\begin{array}{l}\text { Sambucus nigra L. (Adoxaceae) } \\
\text { SB084 } \\
\text { NB054 }\end{array}$} & \multirow{2}{*}{$\begin{array}{l}\text { Soc; бузина } \\
\text { (Buzyna) }\end{array}$} & \multirow[t]{2}{*}{ Flowers } & \multirow[t]{2}{*}{ Drink } & Blood pressure & 1 & \\
\hline & & & & $\begin{array}{l}\text { Good for the } \\
\text { stomach }\end{array}$ & 4 & \\
\hline
\end{tabular}


Table 2 Recorded medicinal taxa in Northern and Southern Bukovina. DUR Detailed Use Reports; RO Romanian Hutsuls; UA Ukrainian Hutsuls (Continued)

\begin{tabular}{|c|c|c|c|c|c|c|}
\hline \multirow{2}{*}{$\begin{array}{l}\text { Latin name, family and voucher } \\
\text { specimens }\end{array}$} & \multirow[t]{2}{*}{ Local names } & \multirow[t]{2}{*}{ Used part(s) } & \multirow[t]{2}{*}{ Preparation } & \multirow[t]{2}{*}{ Medicinal Use } & \multicolumn{2}{|c|}{ DUR } \\
\hline & & & & & $\mathrm{RO}$ & UA \\
\hline & & & & $\begin{array}{l}\text { Good for the } \\
\text { kidneys }\end{array}$ & 4 & \\
\hline & & & & $\begin{array}{l}\text { Good for the } \\
\text { urinary tract }\end{array}$ & 4 & \\
\hline & & & Tea & Colds & & 1 \\
\hline & & & & Cough & & 4 \\
\hline & & & & Flu & & 2 \\
\hline & & & & $\begin{array}{l}\text { Good for the } \\
\text { throat }\end{array}$ & & 2 \\
\hline & & & $\begin{array}{l}\text { Infused in } \\
\text { alcohol }\end{array}$ & Cough & & 1 \\
\hline & & Leaves & Dried & $\begin{array}{l}\text { Good for the } \\
\text { heart }\end{array}$ & & 2 \\
\hline \multirow[t]{2}{*}{ Sedum roseum (L.) Scop. (Crassulaceae) } & \multirow[t]{2}{*}{$\begin{array}{l}\text { Червона щітка (Chervona } \\
\text { shchitka) }\end{array}$} & \multirow[t]{2}{*}{ Roots } & \multirow[t]{2}{*}{ Tea } & $\begin{array}{l}\text { Good for the } \\
\text { pancreas }\end{array}$ & & 1 \\
\hline & & & & $\begin{array}{l}\text { Immune } \\
\text { boosting }\end{array}$ & & 1 \\
\hline \multirow[t]{3}{*}{ Solanum tuberosum L. (Solanaceae) } & \multirow{3}{*}{$\begin{array}{l}\text { Cartofi; бараболя; картошка } \\
\text { (Barabolia; kartoshka) }\end{array}$} & \multirow[t]{3}{*}{ Tubers } & \multirow{2}{*}{$\begin{array}{l}\text { Locally applied } \\
\text { (fresh) }\end{array}$} & Fever & $1^{b}$ & 3 \\
\hline & & & & Headache & 3 & \\
\hline & & & Boiled & Cough & & 3 \\
\hline $\begin{array}{l}\text { Sorbus domestica L. } \\
\text { (Rosaceae) } \\
\text { SB055 } \\
\text { NB232 }\end{array}$ & Scoruș & Fruits & $\begin{array}{l}\text { Tea (fresh/ } \\
\text { dried) }\end{array}$ & Blood sugar & 4 & \\
\hline $\begin{array}{l}\text { Stellaria media (L.) Vill. } \\
\text { (Caryophyllaceae) }\end{array}$ & $\begin{array}{l}\text { Мокриця; червець } \\
\text { (Mokrytsia; chervets) }\end{array}$ & Aerial parts & Tea & Cough & & 2 \\
\hline \multirow{11}{*}{$\begin{array}{l}\text { Symphytum officinale L. (Boraginaceae) } \\
\text { SB070 } \\
\text { NB166; NB167; NB184; NB189 }\end{array}$} & \multirow{11}{*}{$\begin{array}{l}\text { Tătăneasă; живокост; гауізь§; } \\
\text { гауїзь§ } \\
\text { (Zhyvvokost; hauiz§; hauiz§) }\end{array}$} & \multirow[t]{9}{*}{ Roots } & $\begin{array}{l}\text { Locally Applied } \\
\text { (boiled) }\end{array}$ & $\begin{array}{l}\text { Good for the } \\
\text { skin }\end{array}$ & 1 & \\
\hline & & & \multirow{5}{*}{$\begin{array}{l}\text { Locally applied } \\
\text { (fresh) }\end{array}$} & Fracture & 1 & 1 \\
\hline & & & & Gout & & 1 \\
\hline & & & & Joint pain & 1 & 3 \\
\hline & & & & $\begin{array}{l}\text { Rheumatic } \\
\text { pains }\end{array}$ & & 1 \\
\hline & & & & Hernia & 1 & \\
\hline & & & \multirow{2}{*}{$\begin{array}{l}\text { Locally applied } \\
\text { (infused in } \\
\text { alcohol) }\end{array}$} & Joint pain & 2 & \\
\hline & & & & $\begin{array}{l}\text { Good for the } \\
\text { liver }\end{array}$ & 2 & \\
\hline & & & $\begin{array}{l}\text { Locally applied } \\
\text { (fresh with wax) }\end{array}$ & Joint pain & & 1 \\
\hline & & \multirow[t]{2}{*}{ Whole plant } & \multirow[t]{2}{*}{ Tea (fresh) } & $\begin{array}{l}\text { Good for the } \\
\text { liver }\end{array}$ & 2 & \\
\hline & & & & $\begin{array}{l}\text { Good for the } \\
\text { stomach }\end{array}$ & 2 & \\
\hline \multirow{3}{*}{$\begin{array}{l}\text { Syringa vulgaris L. } \\
\text { (Oleaceae) } \\
\text { NB208; NB209 }\end{array}$} & \multirow[t]{3}{*}{ Бузок (Busok) } & \multirow[t]{3}{*}{ Flowers } & $\begin{array}{l}\text { Infused with } \\
\text { moonshine }\end{array}$ & Joint pain & & 1 \\
\hline & & & Tea & Bronchitis & & 1 \\
\hline & & & & Cough & & 3 \\
\hline $\begin{array}{l}\text { Tagetes erecta L. } \\
\text { (Asteraceae) }\end{array}$ & Чорнобривці (Chornobryvtsi) & Flowers & Tea & $\begin{array}{l}\text { Blood } \\
\text { cleansing }\end{array}$ & & 1 \\
\hline
\end{tabular}


Table 2 Recorded medicinal taxa in Northern and Southern Bukovina. DUR Detailed Use Reports; RO Romanian Hutsuls; UA Ukrainian Hutsuls (Continued)

\begin{tabular}{|c|c|c|c|c|c|c|}
\hline \multirow{2}{*}{$\begin{array}{l}\text { Latin name, family and voucher } \\
\text { specimens }\end{array}$} & \multirow[t]{2}{*}{ Local names } & \multirow[t]{2}{*}{ Used part(s) } & \multirow[t]{2}{*}{ Preparation } & \multirow[t]{2}{*}{ Medicinal Use } & \multicolumn{2}{|c|}{ DUR } \\
\hline & & & & & $\mathrm{RO}$ & UA \\
\hline & & & & Abscesses & & 1 \\
\hline & & & & Diabetes & & 1 \\
\hline & & & & $\begin{array}{l}\text { Good for the } \\
\text { liver }\end{array}$ & & 1 \\
\hline \multirow{2}{*}{$\begin{array}{l}\text { Tanacetum balsamita } \mathrm{L} \text {. } \\
\text { (Asteraceae) }\end{array}$} & \multirow[t]{2}{*}{ Canufar; Кануфер (Kanufer) } & \multirow[t]{2}{*}{ Aerial parts } & \multirow{2}{*}{$\begin{array}{l}\text { Infused in } \\
\text { alcohol }\end{array}$} & Abscesses & & 1 \\
\hline & & & & Wounds & $1^{\mathrm{b}}$ & 1 \\
\hline \multirow{3}{*}{$\begin{array}{l}\text { Taraxacum officinale } \\
\text { Webb (Asteraceae) } \\
\text { SB063 } \\
\text { NB016;NB048 }\end{array}$} & \multirow[t]{3}{*}{ Păpădie ; кульбаба (Kulbaba) } & Aerial parts & Raw & Vitamins & & 2 \\
\hline & & Flowers & Syrup (fresh) & $\begin{array}{l}\text { Good for the } \\
\text { liver }\end{array}$ & 1 & \\
\hline & & & Jam & $\begin{array}{l}\text { Good for the } \\
\text { urinary tract }\end{array}$ & 4 & \\
\hline \multirow{15}{*}{$\begin{array}{l}\text { Thymus spp. including } \\
\text { Thymus serpyllum L. and Thymus } \\
\text { vulgaris L. } \\
\text { (Lamiaceae) } \\
\text { SB001; SB090 } \\
\text { NB030; NB027; NB125; } \\
\text { NB019 }\end{array}$} & \multirow{15}{*}{$\begin{array}{l}\text { чабер; чебрець, чебрик; городній чебрець } \\
\text { (Chaber; chebrets, chebryk; horodnii chebrets) } \\
\text { Thymus serpyllum: Cimbrişor; чебрець } \\
\text { звичайний, чебрик польовий; польовий } \\
\text { чебрець (chebrek polovyi; chebrets zvychainyi; } \\
\text { chebryk polovyi; polovyi chebrets) } \\
\text { Thymus vulgaris: } \\
\text { Cimbru sălbatic; чеберець садовий (Cheberets } \\
\text { sadovyi) }\end{array}$} & Aerial parts & Tea & $\begin{array}{l}\text { Good for the } \\
\text { stomach }\end{array}$ & 1 & 2 \\
\hline & & & & Lung diseases & & 1 \\
\hline & & & & $\begin{array}{l}\text { Good for the } \\
\text { throat }\end{array}$ & & 1 \\
\hline & & & & $\begin{array}{l}\text { Good for the } \\
\text { lungs }\end{array}$ & 3 & \\
\hline & & & & Colds & 2 & \\
\hline & & & & Pain & 2 & \\
\hline & & & & Panacea & 3 & \\
\hline & & & & $\begin{array}{l}\text { Good for the } \\
\text { kidneys }\end{array}$ & 1 & \\
\hline & & & Syrup & Cough & & 3 \\
\hline & & & Tea & $\begin{array}{l}\text { Good for } \\
\text { breathing }\end{array}$ & & $1^{\mathrm{b}}$ \\
\hline & & & & Cough & 10 & 12 \\
\hline & & & & Good for veins & 2 & \\
\hline & & & & Alcoholism & & 1 \\
\hline & & & $\begin{array}{l}\text { Burnt three } \\
\text { times }\end{array}$ & Evil Eye & & $1^{b}$ \\
\hline & & Flowers & Tea & Colds & & 1 \\
\hline \multirow{9}{*}{$\begin{array}{l}\text { Tilia cordata Mill. } \\
\text { (Malvaceae) } \\
\text { SB017 } \\
\text { NB253 }\end{array}$} & \multirow[t]{9}{*}{ Теi; липа (Lypa) } & \multirow[t]{9}{*}{ Flowers } & \multirow[t]{9}{*}{ Tea (dried) } & $\begin{array}{l}\text { Good for the } \\
\text { heart }\end{array}$ & 4 & \\
\hline & & & & $\begin{array}{l}\text { Abdominal } \\
\text { pain }\end{array}$ & 1 & \\
\hline & & & & $\begin{array}{l}\text { Good for } \\
\text { digestion }\end{array}$ & 2 & \\
\hline & & & & $\begin{array}{l}\text { Good for the } \\
\text { liver }\end{array}$ & & 2 \\
\hline & & & & $\begin{array}{l}\text { Good for the } \\
\text { stomach }\end{array}$ & 7 & \\
\hline & & & & Fever & & 2 \\
\hline & & & & $\begin{array}{l}\text { Inflammation } \\
\text { processes }\end{array}$ & & 1 \\
\hline & & & & $\begin{array}{l}\text { Organism } \\
\text { cleansing }\end{array}$ & 1 & \\
\hline & & & & Good for & 1 & \\
\hline
\end{tabular}


Table 2 Recorded medicinal taxa in Northern and Southern Bukovina. DUR Detailed Use Reports; RO Romanian Hutsuls; UA Ukrainian Hutsuls (Continued)

\begin{tabular}{|c|c|c|c|c|c|c|}
\hline \multirow{2}{*}{$\begin{array}{l}\text { Latin name, family and voucher } \\
\text { specimens }\end{array}$} & \multirow[t]{2}{*}{ Local names } & \multirow[t]{3}{*}{ Used part(s) } & \multirow[t]{2}{*}{ Preparation } & \multirow[t]{2}{*}{ Medicinal Use } & \multicolumn{2}{|c|}{$\underline{\text { DUR }}$} \\
\hline & & & & & $\mathrm{RO}$ & UA \\
\hline & & & & women & & \\
\hline & & & & $\begin{array}{l}\text { Good for the } \\
\text { kidneys }\end{array}$ & & 2 \\
\hline & & & & Calming & 6 & $1^{b}$ \\
\hline & & & & Headache & 1 & \\
\hline & & & & Headache & 1 & 1 \\
\hline & & & & Soporific & 2 & \\
\hline & & & & Colds & 2 & 1 \\
\hline & & & & Cough & 3 & 1 \\
\hline & & & & Flu & 1 & \\
\hline & & & & Panacea & & $1^{b}$ \\
\hline & & Leaves & Boiled & Hair care & & 2 \\
\hline $\begin{array}{l}\text { Trifolium pannonicum Jacq. } \\
\text { (Leguminosae) }\end{array}$ & $\begin{array}{l}\text { конюшина панойська з жовтими квітами } \\
\text { (Koniushyna panoiska z zhovtymy kvitamy) }\end{array}$ & Flowers & Tea (dried) & Healthy & & 1 \\
\hline \multirow{3}{*}{$\begin{array}{l}\text { Trifolium sp. including Trifolium pratense } \\
\text { L. } \\
\text { (Leguminosae) } \\
\text { SB072; SB075; SB077; SB078 } \\
\text { NB002; NB013; NB014; NB076; NB086; } \\
\text { NB102; NB103; NB110; NB111; NB112; } \\
\text { NB119; NB123; NB126; NB134; NB140; } \\
\text { NB144 }\end{array}$} & \multirow{3}{*}{$\begin{array}{l}\text { Trifoi alb; Trifoi rosu; тріфоль; конюшина } \\
\text { червона } \\
\text { (Trifol; koniushyna chervona) }\end{array}$} & Aerial parts & Tea (dried) & $\begin{array}{l}\text { Good for the } \\
\text { urinary tract }\end{array}$ & 2 & \\
\hline & & & & Headache & 1 & 1 \\
\hline & & & & $\begin{array}{l}\text { Good for the } \\
\text { lungs }\end{array}$ & 4 & \\
\hline \multirow{6}{*}{$\begin{array}{l}\text { Tussilago farfara L. (Asteraceae) } \\
\text { SB065; SB085 } \\
\text { NB072; NB133 }\end{array}$} & \multirow{6}{*}{$\begin{array}{l}\text { Podbal; підбіл;мати й мачуха; } \\
\text { (Pidbil; maty у machukha) }\end{array}$} & Aerial parts & Tea & Cough & 1 & 9 \\
\hline & & Flowers & Syrup (fresh) & Cough & 1 & \\
\hline & & & Tea & Colds & & 1 \\
\hline & & Leaves & $\begin{array}{l}\text { Locally applied } \\
\text { (fresh) }\end{array}$ & warts & 2 & \\
\hline & & Roots & Syrup & $\begin{array}{l}\text { Good for the } \\
\text { throat }\end{array}$ & & 1 \\
\hline & & Whole plant & Boiled & Cough & & 1 \\
\hline \multirow{12}{*}{$\begin{array}{l}\text { Urtica dioica L. } \\
\text { (Urticaceae) } \\
\text { SB088 } \\
\text { NB026; NB048 }\end{array}$} & \multirow[t]{12}{*}{$\begin{array}{l}\text { Urzică; кропива; кропива жалка (Kropyva; } \\
\text { kropyva zhalka) }\end{array}$} & \multirow[t]{12}{*}{$\begin{array}{l}\text { Young sprouts } \\
\text { (aerial parts) }\end{array}$} & \multirow[t]{2}{*}{ Boiled (in soup) } & $\begin{array}{l}\text { Blood } \\
\text { cleansing }\end{array}$ & 4 & 1 \\
\hline & & & & $\begin{array}{l}\text { Vessel } \\
\text { cleansing }\end{array}$ & & 1 \\
\hline & & & \multirow[t]{10}{*}{ Tea } & $\begin{array}{l}\text { Blood } \\
\text { cleansing }\end{array}$ & 9 & 3 \\
\hline & & & & Blood pressure & & 1 \\
\hline & & & & $\begin{array}{l}\text { Good for the } \\
\text { heart }\end{array}$ & 1 & 2 \\
\hline & & & & $\begin{array}{l}\text { Good for the } \\
\text { stomach }\end{array}$ & & 2 \\
\hline & & & & Vomiting & & 2 \\
\hline & & & & $\begin{array}{l}\text { Rheumatic } \\
\text { pains }\end{array}$ & 2 & \\
\hline & & & & Calming & & 2 \\
\hline & & & & Toothache & & 2 \\
\hline & & & & Nosebleeds & & 2 \\
\hline & & & & Healthy & 1 & \\
\hline
\end{tabular}


Table 2 Recorded medicinal taxa in Northern and Southern Bukovina. DUR Detailed Use Reports; RO Romanian Hutsuls; UA Ukrainian Hutsuls (Continued)

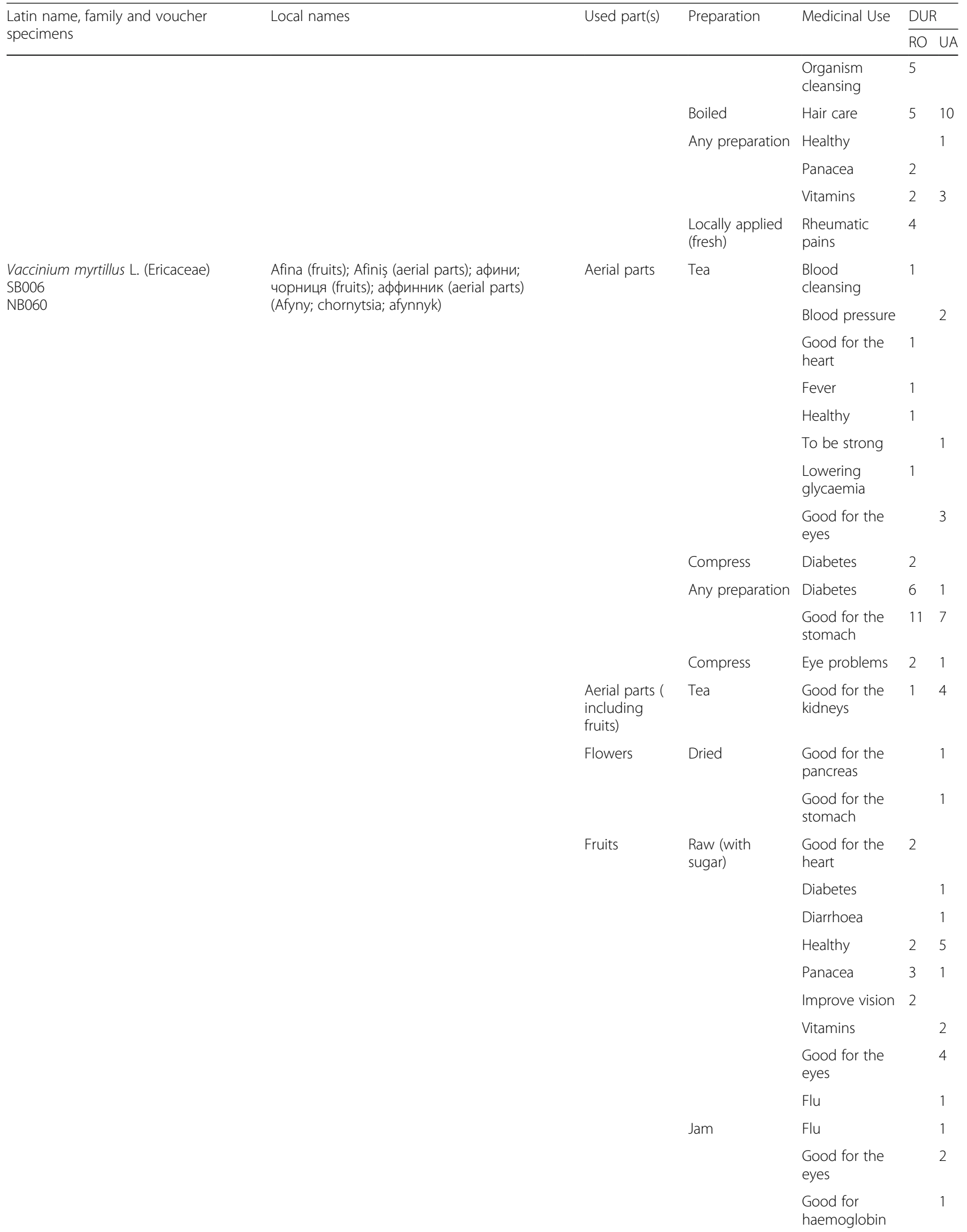


Table 2 Recorded medicinal taxa in Northern and Southern Bukovina. DUR Detailed Use Reports; RO Romanian Hutsuls; UA Ukrainian Hutsuls (Continued)

\begin{tabular}{|c|c|c|c|c|c|c|}
\hline \multirow{2}{*}{$\begin{array}{l}\text { Latin name, family and voucher } \\
\text { specimens }\end{array}$} & \multirow[t]{2}{*}{ Local names } & \multirow[t]{2}{*}{ Used part(s) } & \multirow[t]{2}{*}{ Preparation } & \multirow[t]{2}{*}{ Medicinal Use } & \multicolumn{2}{|c|}{ DUR } \\
\hline & & & & & $\mathrm{RO}$ & UA \\
\hline & & & & Diarrhoea & & 1 \\
\hline & & & Juice & Diarrhoea & 1 & \\
\hline & & & & $\begin{array}{l}\text { Good for the } \\
\text { liver }\end{array}$ & 1 & \\
\hline & & & Syrup & $\begin{array}{l}\text { Abdominal } \\
\text { pain }\end{array}$ & 1 & \\
\hline & & & & $\begin{array}{l}\text { Good for the } \\
\text { abdomen }\end{array}$ & 1 & \\
\hline & & & & Diarrhoea & 1 & 3 \\
\hline & & & & $\begin{array}{l}\text { Appetite } \\
\text { suppressant }\end{array}$ & 1 & \\
\hline & & & & Panacea & & 2 \\
\hline & & & & $\begin{array}{l}\text { Good for the } \\
\text { liver }\end{array}$ & 2 & \\
\hline & & & $\begin{array}{l}\text { Infused raw in } \\
\text { alcohol }\end{array}$ & $\begin{array}{l}\text { Good for the } \\
\text { stomach }\end{array}$ & & 4 \\
\hline & & & & $\begin{array}{l}\text { Stomach } \\
\text { diseases }\end{array}$ & & 2 \\
\hline & & & & 100 diseases & & 6 \\
\hline & & & & $\begin{array}{l}\text { Good for the } \\
\text { eyes }\end{array}$ & & 2 \\
\hline & & & & Stomach pain & & 2 \\
\hline & & & Dried & 100 diseases & & 2 \\
\hline & & & Dried & $\begin{array}{l}\text { Good for the } \\
\text { eyes }\end{array}$ & & 2 \\
\hline & & & Tea & Panacea & & 2 \\
\hline & & & & $\begin{array}{l}\text { Good for the } \\
\text { eyes }\end{array}$ & & 3 \\
\hline & & & & Diarrhoea & & 6 \\
\hline & & & & Improve vision & 1 & \\
\hline & & & Tincture & Flu & & 1 \\
\hline \multirow{11}{*}{$\begin{array}{l}\text { Vaccinium vitis-idaea L. (Ericaceae) } \\
\text { SB010 } \\
\text { NB061 }\end{array}$} & \multirow{11}{*}{ 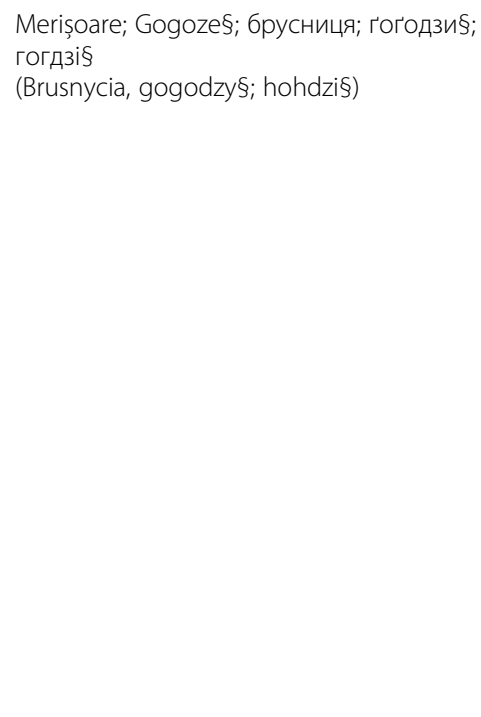 } & \multirow[t]{9}{*}{ Aerial parts } & Tea & $\begin{array}{l}\text { Good for the } \\
\text { heart }\end{array}$ & 1 & \\
\hline & & & & Diabetes & & 1 \\
\hline & & & & Diarrhoea & 1 & \\
\hline & & & & $\begin{array}{l}\text { Good for the } \\
\text { liver }\end{array}$ & 1 & \\
\hline & & & & $\begin{array}{l}\text { Good for the } \\
\text { stomach }\end{array}$ & 1 & \\
\hline & & & & Fever & 1 & \\
\hline & & & & $\begin{array}{l}\text { Good for the } \\
\text { kidneys }\end{array}$ & 1 & 5 \\
\hline & & & & $\begin{array}{l}\text { Kidney } \\
\text { diseases }\end{array}$ & 1 & \\
\hline & & & & $\begin{array}{l}\text { Urinary tract } \\
\text { diseases }\end{array}$ & 1 & \\
\hline & & \multirow[t]{2}{*}{ Fruits } & Any preparation & Blood pressure & & 3 \\
\hline & & & Raw & Blood pressure & & 1 \\
\hline
\end{tabular}


Table 2 Recorded medicinal taxa in Northern and Southern Bukovina. DUR Detailed Use Reports; RO Romanian Hutsuls; UA Ukrainian Hutsuls (Continued)

\begin{tabular}{|c|c|c|c|c|c|c|}
\hline \multirow{2}{*}{$\begin{array}{l}\text { Latin name, family and voucher } \\
\text { specimens }\end{array}$} & \multirow[t]{2}{*}{ Local names } & \multirow[t]{2}{*}{ Used part(s) } & \multirow[t]{2}{*}{ Preparation } & \multirow[t]{2}{*}{ Medicinal Use } & \multicolumn{2}{|c|}{ DUR } \\
\hline & & & & & $\overline{\mathrm{RO}}$ & $\overline{U A}$ \\
\hline & & & & Panacea & & 1 \\
\hline & & & & Vitamins & & 4 \\
\hline & & & & $\begin{array}{l}\text { Good for the } \\
\text { kidneys }\end{array}$ & & 3 \\
\hline & & & & Urolithiasis & & 1 \\
\hline & & & & $\begin{array}{l}\text { Good for the } \\
\text { heart }\end{array}$ & 1 & \\
\hline & & & Tea & Blood pressure & 1 & \\
\hline & & & & Panacea & & 2 \\
\hline & & & & $\begin{array}{l}\text { Good for the } \\
\text { heart }\end{array}$ & 1 & 1 \\
\hline & & & Compote & Fever & & 1 \\
\hline & & & Juice & Fever & 1 & \\
\hline & & & $\begin{array}{l}\text { Syrup (without } \\
\text { sugar) }\end{array}$ & Fever & 1 & \\
\hline & & & $\begin{array}{l}\text { Water source } \\
\text { and fresh fruits/ } \\
\text { compote }\end{array}$ & $\begin{array}{l}\text { Immune } \\
\text { boosting }\end{array}$ & 1 & 1 \\
\hline & & & Compote & Cough & & 1 \\
\hline & & & Any preparation & Flu & & 1 \\
\hline & & Roots & Tea & $\begin{array}{l}\text { Good for the } \\
\text { bladder }\end{array}$ & & 1 \\
\hline Valeriana officinalis L. (Caprifoliaceae) & Валеріана (Valeriana) & Roots & $\begin{array}{l}\text { Infused in } \\
\text { alcohol }\end{array}$ & Heart disease & & 1 \\
\hline \multirow{14}{*}{$\begin{array}{l}\text { Viburnum opulus L. (Adoxaceae) } \\
\text { NB223 }\end{array}$} & \multirow[t]{14}{*}{ Călină; калина (Kalyna) } & Flowers & Tea & Fever & & 2 \\
\hline & & Fruits & Tea & Blood pressure & & 7 \\
\hline & & & & $\begin{array}{l}\text { Good for the } \\
\text { heart }\end{array}$ & 4 & \\
\hline & & & & Panacea & 2 & \\
\hline & & & & Cold & 2 & \\
\hline & & & & Cough & 4 & \\
\hline & & & & $\begin{array}{l}\text { Good for the } \\
\text { lungs }\end{array}$ & 4 & \\
\hline & & & & Fever & & 2 \\
\hline & & & Syrup & Blood pressure & & 2 \\
\hline & & & & Flu & & 1 \\
\hline & & & & Fever & & 1 \\
\hline & & & $\begin{array}{l}\text { Raw (with } \\
\text { sugar) }\end{array}$ & $\begin{array}{l}\text { Good for the } \\
\text { heart }\end{array}$ & & 1 \\
\hline & & & & Panacea & & 1 \\
\hline & & Leaves & Boiled & Joint pain & & 1 \\
\hline \multirow{2}{*}{$\begin{array}{l}\text { Vitis vinifera L. } \\
\text { (Vitaceae) } \\
\text { NB204 }\end{array}$} & \multirow[t]{2}{*}{ Виноград (Vynohrad) } & \multirow[t]{2}{*}{ Fruits } & \multirow[t]{2}{*}{ Wine } & $\begin{array}{l}\text { Good for } \\
\text { blood }\end{array}$ & & 1 \\
\hline & & & & Panacea & & 1 \\
\hline
\end{tabular}

Plant names mentioned by Ukrainian Hutsuls are reported in Cyrillic (with transliteration). Plant names mentioned by Romanian Hutsuls are reported in the Latin alphabet. Plant names not reported in Romanian or Ukrainian dictionaries or in previous publications (e.g. Pieroni and Soukand, 2017), and are therefore probably Hutsul names, are marked with a §. A Russian name is marked with a $\wedge$

adenotes a recently adopted use

${ }^{b}$ denotes a past use 
a.

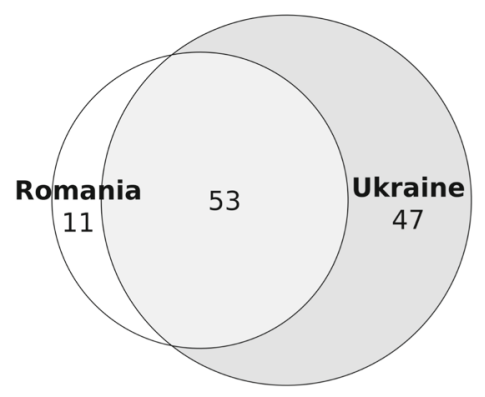

b.

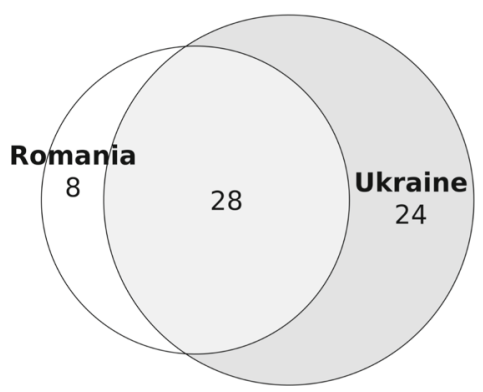

Fig. 5 a The proportional Venn diagram shows that the majority of mentioned medicinal taxa were common to Hutsuls living in Northern and Southern Bukovina. However, a very large number of taxa were reported only in Ukraine; $\|=48$. b The proportional Venn diagram of medicinal taxa mentioned by at least three interviewees shows that Romanian Hutsuls used medicinal plants more consistently than Ukrainian Hutsuls. Indeed, 23 taxa were mentioned by only one or two Ukrainian Hutsuls. This result is in line with the findings regarding the use of food taxa.; $J$ $=46$

Romanian Hutsuls Ukrainian Hutsuls

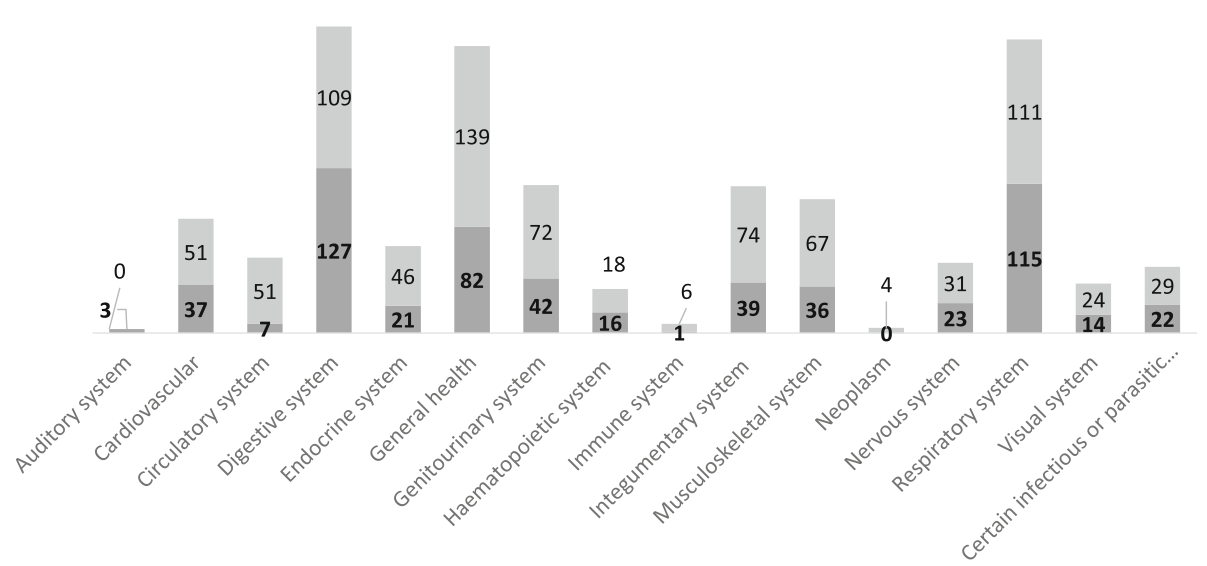

Fig. 6 The distribution of medicinal DUR for the ICD-11 system categories shows that general health was the most important category among Ukrainian Hutsuls, while the digestive system was the most reported among Romanian Hutsuls. Both groups reported treating disorders of the respiratory system with medicinal plant preparations 
a

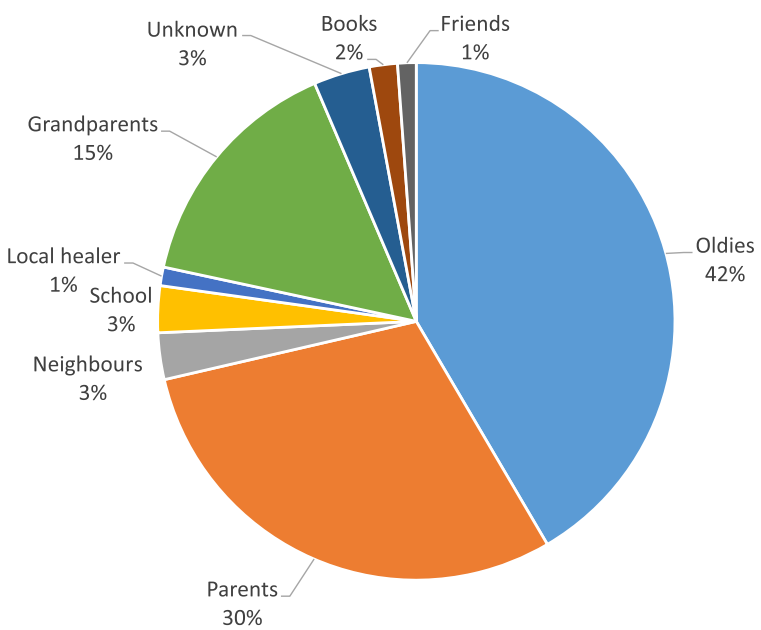

b

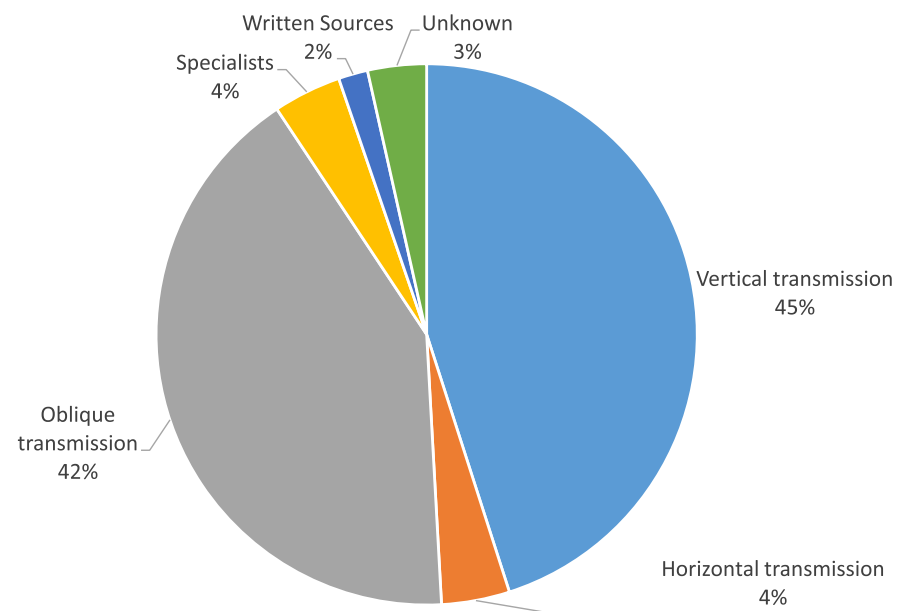

Fig. 7 a Ecological knowledge transmission strategies among Romanian Hutsuls. b Ecological knowledge transmission categories among Romanian Hutsuls grouped per strategy

there was always a lot of work in maintaining their smallscale farms. Moreover, all the Romanian people who mentioned books as a source of knowledge added that they would never have trusted this information as such, but they had a solid base of knowledge derived from oral sources and they have just added some information to it (for instance, they did not know a specific plant was useful for something, but they were already using it or part of it).

Among the Ukrainian Hutsuls, we recorded nearly the same proportion of vertical ecological knowledge transmission from parents and grandparents (48\%), as well as the same amount of horizontal transmission from neighbours and oblique transmission from local elderly individuals (11\%) (Fig. 8). We also observed that $15 \%$ of knowledge was obtained from written sources including books and newspapers ('I read in the newspaper that a bath with
Chelidonium majus and Matricaria chamomilla helps with allergies', explained a women born in 1969), 6\% from the Internet and $2 \%$ from television.

Comparing the two communities, we can observe that the elderly, and thus oblique LEK transmission, play a minor role among Ukrainian Hutsuls, while neighbours have a more important role ('Come to my neighbour, she knows everything', advised an old woman born in 1928). Also, in Ukraine, no one reported having learnt from specialists, while mass media such as the Internet and television accounted for $8 \%$, which added to the $15 \%$ from books and newspapers totals $23 \%$, whereas this value is only $12 \%$ among Romanians.

Moreover, while only one book was mentioned [28] and another one was presented during interviews in Southern Bukovina [30], Ukrainian Hutsuls reported 16 
a

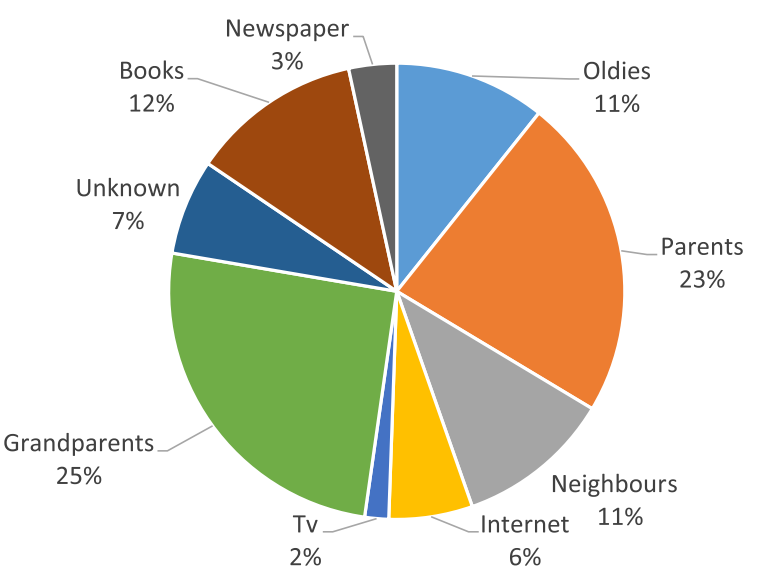

b

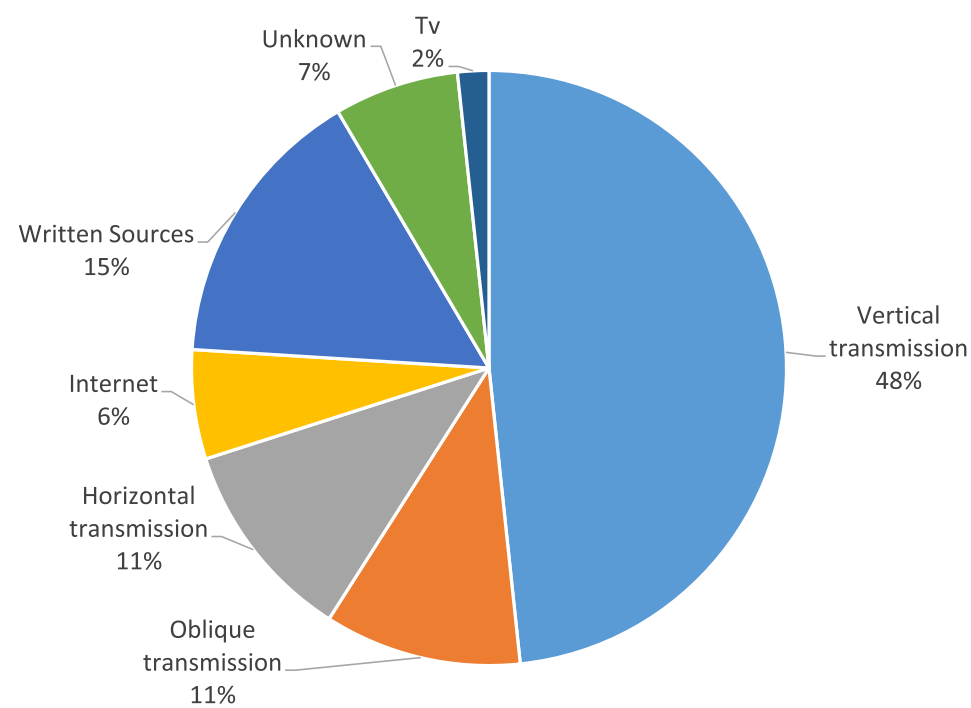

Fig. 8 a Ecological knowledge transmission strategies among Ukrainian Hutsuls. b Ecological knowledge transmission strategies among Ukrainian Hutsuls grouped per strategy

books in both Ukrainian and Russian published between 1979 and 2016 (Table 3).

\section{Popular books about medicinal plants in Northern Bukovina (Ukraine)}

Books on medicinal plants were very popular in Ukraine and could be grouped based on the period of their publication. The first period of mass publication of books on wild medicine began in the 1970s. At that time, most of the books had an official reviewer controlled by Moscow, as a rule a doctor or professor of medicine. The popularity of herbal medicinal books can be seen by the number of editions; for example, Dr. Karhut's 'Medicine around us' was republished in 1975, 1978 and 1979. Hammerman and co- authors published the text book 'Medical plants or planthelpers' in 1978 and then again in 1979 for biological specialties and medical schools, which was adopted by the Ministry of Education of the USSR.

The second period started at the beginning of the 1990s when there were no longer censors, and therefore a boom of book publications took place; and indeed out of the 16 books mentioned during our interviews, 11 are from this period. Besides books, respondents named a variety of newspapers that specialized in recipes of wild and domesticated taxa for medicinal purposes. We recorded eight different newspapers and magazines named by interviewees, e.g. 'Alphabet of health', 'Health advice', 'Good doctor' and 'Granny'. These magazines were very cheap and promoted 
Table 3 Details of the books reported during interviews in Northern Bukovina, Ukraine

\begin{tabular}{|c|c|c|c|c|c|}
\hline Author & Year & Title & Publisher/City & Language & $\begin{array}{l}\text { No of } \\
\text { pages }\end{array}$ \\
\hline Alekseev I. Dibrova A. & 2012 & Complete atlas of medicinal plants & Gloria, Kiev & Russian & 400 \\
\hline Grechanyi I. & 2015 & The Great Illustrated Directory of Medicinal Herbs & Book club 'Family Leisure Club', Kharkiv & Ukrainian & 544 \\
\hline Grodzinsky AM. & 1990 & Medicinal plants: Encyclopedic reference book & $\begin{array}{l}\text { 'Ukrainska encuklopedia' MP Bazhana, } \\
\text { Kiev }\end{array}$ & Ukrainian & 544 \\
\hline Markova A. & 2002 & The Complete Encyclopedia of Folk Medicine & Esmo, Moscow & Russian & 640 \\
\hline Pavlenko L. & 1992 & Drugs from Chardzilla & Veselka, Kyiv & Ukrainian & 52 \\
\hline Reutov S. & 2016 & Natural healers of 1000 diseases & Book club 'Family Leisure Club', Kharkiv & Russian & 320 \\
\hline $\begin{array}{l}\text { Rosola T. Rosola I. Rubish } \\
\text { F. }\end{array}$ & 2012 & $\begin{array}{l}\text { Medicinal plants of Transcarpathia in folk } \\
\text { medicine }\end{array}$ & Patent, Uzgorod & Ukrainian & 208 \\
\hline $\begin{array}{l}\text { Ivashyn D. Katina Z. } \\
\text { Rybachuk I. et al. }\end{array}$ & 1983 & $\begin{array}{l}\text { Directory of preparations of medicinal plants } \\
\text { harvest }\end{array}$ & Urozai, Kev & Russian & 296 \\
\hline Safonov MM. & 2015 & Full atlas of medicinal plants & Bogdan, Ternopil' & Ukrainian & 384 \\
\hline Schultz J. Uberguber E. & 1994 & Medicines from God's Pharmacy & Anfas, Kiev & Russian & 207 \\
\hline Smik GK. & 1991 & Useful and rare plants of Ukraine & Ukrainska radyanska encuklopedia, Kiev & Ukrainian & 416 \\
\hline $\begin{array}{l}\text { Smolinskaya M. } \\
\text { Korolyuk V. Galitska L. }\end{array}$ & 2002 & Medicinal plants of Bukovina & Ruta, Cernivtci & Ukrainian & 295 \\
\hline Sokolov C. Zamotayev I. & 1988 & Directory of Medicinal Plants & Nedra, Moskow & Russian & 464 \\
\hline Uzhegov $\mathrm{H}$. & 2011 & The Complete Encyclopedia of Folk Medicine & Astrel, Moskow & Russian & 1088 \\
\hline Henzel W. & 2016 & An illustrated herbalist. 350 species & Family Leisure Club, Kharkiv & Russian & 256 \\
\hline Yelin Y. & 1979 & Plants of our forests & Soviet School, Kiev & Ukrainian & 239 \\
\hline $\begin{array}{l}\text { Zinchenko TV. } \\
\text { Stakhiv IV. Myakushko T. }\end{array}$ & 1990 & Medicinal plants in gastroenterology & Naukova Dumka, Kiev & Russian & 240 \\
\hline
\end{tabular}

by the state postal service. Those publications included recipes from medical doctors as well as from people that 'treated themselves' with specific remedies.

\section{Different attitudes towards written and visual sources among Hutsuls on the two sides of the border}

We observed a different attitude towards written sources between the two communities. While in Romania books were somehow perceived as unnecessary, not completely useful (as the elderly know more) and not to be trusted (as the elderly know better), in Ukraine they were a real source of pride. 'We are very knowledgeable people, we go to libraries', claimed a woman (born in 1966). Indeed, in Ukraine during the Soviet era, education and books were important ways of showing off, as boasted by a Hutsul woman (born in 1948): 'I have an expensive book! (the medicinal plants book)'. This is because books were very rare and hard to get during Soviet times [31]. Therefore, the large number of books shown during our interviews may be due to informants' pride of being able to show that they are knowledgeable people who have the economic power to buy books and can acquire 'high' knowledge (compared to the lesser importance of oral knowledge). Specifically, books regarding medicinal uses of plants were propagandized and it was a popular topic in schools and universities. In addition, phytotherapeutic knowledge was especially sought-after because the Soviet medical system relied heavily on herbal medicine, e.g. a special course on herbal medicine was offered at all medical universities of Soviet Ukraine [32,3 3]. Indeed, this positive attitude towards 'official' and written sources has been observed in other post-Soviet countries and confirms that book knowledge is considered especially trustworthy in these contexts [32].

Another difference between Ukrainian and Romanian Hutsuls is that neighbours are an important source of knowledge among the former, while a similar role is played by the elderly among the latter. Although it may simply be a phenomenon related to semantics (elderly individuals can also be neighbours), there may be more older and knowledgeable people in Romania, as in Ukraine a particular generation was deported to Siberia and never returned, or if individuals did return they did not live long [33] or were killed during WWII and the time of repressions [34].

\section{Different perspectives on Hutsul ecological knowledge transmission patterns on both sides of the border}

Our overall data reveals that LEK among Romanian and Ukrainian Hutsuls is transferred using different transmission patterns and sources. Indeed, among 
Romanians, the main rule seemed to be the experiential 'uite, asta-i buna sau nu-i buna (look, this is good, this is not)' learnt from parents or the elderly of the village, as an 85-year-old Hutsul man reported. This attitude is clearly encompassed by the definition of traditional knowledge, as it is transmitted orally in the local language and characterized by ubiquitous dissemination. The other sources of knowledge accounted for only $6 \%$ in total.

Among Ukrainian Hutsuls, there is a larger proportion of knowledge that comes from other sources (23\%). However, even though magazines and pamphlets were found to be an important source of knowledge in several post-Soviet countries [35, 36], the Internet and television were not found to influence medicinal plant knowledge in other areas of Ukraine [37].

\section{Socio-political factors affecting LEK in Northern Bukovina (Ukraine)}

The reasons for the different knowledge transmission strategies may be found in the distinct social and political environments which the 'new' border created. In Northern Bukovina, Hutsuls were part of a centripetal system that delivered services and information equally to every part of the USSR.

The educational system promoted by the Soviet Union significantly impacted the Hutsul way of thinking and living [38]. All across Ukraine, this was implemented through both the mandatory teaching of the Russian language, which was required for any prestigious job [39], and the promotion of 'rural clubs', which proposed new forms of political education such as mobile libraries and cinemas in order to reach people in even very remote villages [39]. This kind of policy aimed to prevent the expression of local (Hutsul) identity by fostering the assimilation of Soviet culture in the Ukrainian territory [40]. Among others, the Soviet regime targeted the expression of Hutsul identity and many traditions and rituals were banned. For instance, wearing Hutsul clothing and singing traditional songs were not allowed [41]. The traditional (religious) calendar was altered and only events devoid of any identitarian features were maintained [41].

The social landscape of Ukrainian Hutsuls abruptly changed in the 1940s when, concomitantly with border creation, drastic depopulation and the collectivization of farms and arable land occurred [42]. Indeed, despite the meagre amount of arable land in the Carpathian valleys, many collective farms were established there, and in the area of Putyla as well ('There were collective farms and it was hard to live. I have been working since I was 14', mentioned an elderly individual). Several interviewees reported that there were important wool factories, which benefitted from the large number of sheep present in this area of the Carpathians, in addition to the centralized management of the forest and the promotion of rural clubs ('Can you believe there was a cinema here?', asserted a middle-aged male informant).

\section{Socio-political factors affecting LEK in Southern Bukovina (Romania)}

In Southern Bukovina, beginning in the 1960s, the Romanian government promoted rural systematization ('sistematizarea') in order to foster the reconciliation of differences between urban and rural settlements [43]. However, in the following decade, the government recognised the difficulty of rural systematization in the Carpathians, its limited economic potential and the existence of various difficulties, which were sociological, geographical and ethnographical in nature. Therefore, in the 1980s when the main priority turned to agriculture, the project of rural systematization in the Carpathian Mountains was definitively abandoned [43]. In support of this thesis, some local interviewees reported not having experienced the collective farms (otherwise widespread in Romania), due to the limited agricultural productivity of the area. Moreover, local interviewees claimed that livestock and game used to belong to the State, but due to the vastness of the area, the harshness of the steep terrain and communication difficulties, there was not much control in the mountains where Hutsuls live. Therefore, the peripheral location of the area with regard to Romania, as well as its lying along the border and its ethnolinguistic peculiarity prevented this area from being subjected to the centralization policies implemented throughout most of the country (in fact, Romanian Hutsuls reported that only between the 1960s and 1989 were the local forests managed by the central government). As a consequence, ethnobotanical knowledge among Romanian Hutsuls was mainly maintained through vertical transmission (as other sources of knowledge were not widely available).

\section{The effects of these different socio-political contexts on medicinal LEK}

Therefore, the creation of the border and the consequent socio-political contexts unevenly affected the LEK of Romanian and Ukrainian Hutsuls, despite a common ethnolinguistic background, very similar environmental conditions and the peripherality of these areas in their respective geopolitical contexts. Indeed, in Romania, the area in which Hutsuls live was considered remote and of limited economic interest and as a result left behind in the implementation of the 'sistematizarea'. In Ukraine, the centripetal power of Moscow was stronger and thus eliminated the concept of peripherality. The reforms were indeed implemented with the same intensity throughout Soviet territory, and the Russian language and collective farms were imposed. 
The different success of the policies of the Soviet and Romanian regimes, therefore, differently affected Hutsul LEK. While Romanian Hutsul LEK appears to have been somehow 'frozen/static' during the twentieth century, as they were not systematically affected by centralization policies or other factors, Ukrainian Hutsuls were strongly influenced by the new language (Russian) which served as a vector for new (and sometimes technical) knowledge, including the transmission of plant knowledge especially through books and newspapers. Therefore, in addition to vertical knowledge transmission among Ukrainian Hutsuls, we found that other sources of knowledge played an important role. As described in Table 4, these two kinds of LEK sources differ especially with regard to geographical range: while TEK is strictly situational and local and may vary from village to village, other sources may have a wider geographical range, thus encompassing some elements foreign to the community but common to other contexts.

\section{Different pathways of medicinal LEK in Northern and Southern Bukovina}

Our analysis highlights different trends for food and medicinal LEK among Ukrainian and Romanian Hutsuls. While food uses were quantitatively and qualitatively comparable, about $30 \%$ more medicinal uses were reported among Ukrainian Hutsuls. We consider that this might be due to the low availability of physicians and long distances in the sparsely inhabited Hutsul valleys (despite official statistics reporting 3.51 physicians per 1000 inhabitants in Ukraine versus 1.47 in Romania in 1980, [44]), as well as the unavailability of synthetic drugs in health centres.

The higher number of medicinal plants may also be a reaction to Soviet policies which promoted allopathic medicines, discouraging traditional plant-based medicines [35]; for example, a middle-aged Ukrainian women fiercely claimed 'My mother is 77 years old and has never used a single pill in her life', and also another women who stated 'Listen to what is said about medicinal plants so that you do not get sick and do not have to take pills. We drink teas made from Carpathian herbs'. This phenomenon may have been fostered by the severe economic crisis which affected Ukraine after the collapse of the Soviet Union. Indeed, we observed that during this period, medicinal plants were highly promoted by mass media and books; out of the 16 books Ukrainian Hutsuls showed us, 11 were published in this period (1990s).

In the Romania of Ceaușescu, 'everyone had the right to be hospitalized', agreed a middle-age couple; however, a younger male interviewee (born in 1974) also reported that 'at that time (when I was child) there were no doctors, no roads, but there were people who knew plants', which was confirmed by an older Hutsul woman (born in 1927) who stated 'when I arrived here (from Ukrainian Bukovina, after border creation), I learnt everything from a local healer and my neighbour. All I knew at the time I came here was the plants we had to harvest for the army during school hours. Among them I remember arnica'. Therefore, it follows that medicinal knowledge in Romania was to some extent ubiquitous, although some local healers held more (maybe also literary) knowledge and were considered reference points within the Hutsul community.

We could not obtain the source of knowledge for each plant, but we can identify some pan-Soviet elements which were not found on the Romanian side of the border. Indeed, we can observe some of the consequences of the reforms implemented in the Soviet era such as the cultivation of Panax gingseng, Ginkgo biloba, Aloe vera, Aronia melanocarpa and Elaeagnus rhamnoides and their medicinal uses. Specifically, Aronia melanocarpa gained popularity in the late 1940s when the Soviet Union started large-scale cultivations for the production of juices and jams. However, it was also used as herbal medicine, especially as an antihypertensive and anti-atherosclerotic, in several countries of Eastern Europe including Ukraine [45]. Another example of LEK of pan-Soviet origin is the use of Elaeagnus rhamnoides, whose industry, just as with Aronia melanocarpa, grew in the 1940s. Its oil was reported in the Russian Pharmacopeia as an anti-inflammatory [46].

As observed by Fedorak [47], despite several changes Bukovina has faced since Austro-Hungarian times, Hutsuls have fiercely strived to maintain their culture, which

Table 4 Characteristics of knowledge sources among Bukovinian Hutsuls

\begin{tabular}{|c|c|c|}
\hline Characteristics & Knowledge mainly orally transmitted & $\begin{array}{l}\text { Knowledge in which borders between written and oral forms of knowing } \\
\text { nature and practicing this knowledge are more blurred }\end{array}$ \\
\hline Language & $\begin{array}{l}\text { Mainly local language (Hutsul) but also official } \\
\text { languages (Romanian and Ukrainian) }\end{array}$ & $\begin{array}{l}\text { Mainly official languages (Romanian and Ukrainian) but also foreign languages } \\
\text { (Russian) }\end{array}$ \\
\hline Accessibility & Widely accessible within the village & Not necessarily available within the village. \\
\hline $\begin{array}{l}\text { Geographical } \\
\text { range }\end{array}$ & $\begin{array}{l}\text { Strictly situational and local, sometimes varies from } \\
\text { village to village }\end{array}$ & Large geographical ranges (often defined by official language expansion) \\
\hline $\begin{array}{l}\text { Ingredients } \\
\text { used }\end{array}$ & Always local or easily attainable & Not necessarily local \\
\hline
\end{tabular}


has been possible, in part, to their scattered dwellings and the remoteness of the mountains. However, the creation of the border resulted in different socio-political circumstances which affected Hutsul LEK in different ways on each side of the border.

Finally, more and more people have resorted to frequenting pharmacies, probably also fostered by globalization and increased economic means (especially among Romanian Hutsuls, who are now European Union citizens). This trend was observed among both Romanian and Ukrainian Hutsuls who often answer to our questions 'now everyone goes to the pharmacy'.

\section{Conclusions}

We found a total of 118 food and medicinal plants from 107 genera and 53 families. Among Hutsuls of Northern Bukovina we recorded 107 taxa, while there were 72 taxa among Hutsuls of Southern Bukovina. The most used plants were the same in both communities: Vaccinium myrtillus, Rubus idaeus and Urtica dioica.

Despite a common cultural and linguistic background, the ethnobotanical knowledge transmission occurs in different ways on each side of the border. Family is a primary source of ethnobotanical knowledge transmission on both sides of the border; however, in Romania, knowledge from other sources is very limited, whereas in Ukraine interviewees reported several other sources including books, magazines, newspapers, the Internet and television. Indeed, this is especially evident when analysing the wild plants used for medicinal purposes. While recorded food uses are comparable in the two Hutsul communities, our overall data show a disparity regarding the medicinal use of plant taxa. Ukrainian Hutsuls reported around 30\% more plant taxa than Romania Hutsuls. The latter group mentioned almost exclusively locally available plants, whereas the former group reported some plants not mentioned by Romanians such as Aloe vera, Maclura pomifera and Aronia melanocarpa. Knowledge regarding these plants was probably not transferred vertically, within the same family, but by other sources of knowledge such as books, newspapers, magazines and possibly radio, as a consequence of the policies implemented during the Soviet era, including the widespread promotion of Russian language and culture, as well as allopathic drugs. Therefore, this may imply hybridization of the local body of knowledge with foreign elements originating in the Soviet context which has enriched the corpus of ethnobotanical knowledge held by Ukrainian Hutsuls.

Further research should specifically address the plant taxa recently introduced in the body of LEK of Ukrainian Hutsuls in order to understand how such knowledge was conveyed and absorbed by Hutsul mountain communities.

\section{Abbreviations}

DUR: Detailed use report(s); J: Jaccard Index; ICD: International Classification of Diseases; LEK: Local ecological knowledge; RO: Romania; TEK: Traditional ecological knowledge; UA: Ukraine; USSR: Union of Soviet Socialist Republics; WWII: Second World War

\section{Acknowledgements}

We are very grateful to all the Hutsuls who kindly shared their knowledge and practices with us. We thank Angela and Mișu for their valuable assistance in the field.

\section{Authors' contributions}

RS designed the study, GM and NS conducted the fieldwork and GM analysed the data and drafted the manuscript with major contributions from all the authors. NS performed a first analysis of the food uses and drafted the section regarding Ukrainian book sources and its table. RS and AP supervised the study. All authors read and approved the final manuscript.

\section{Funding}

This project received funding from the European Research Council (ERC) under the European Union's Horizon 2020 research and innovation programme (grant agreement No 714874)

Availability of data and materials

All data are available in this publication.

Ethics approval and consent to participate

We strictly followed the ethical guidelines prescribed by the International Society of Ethnobiology (ISE, 2006).

Consent for publication

Not applicable.

\section{Competing interests}

The authors declare that they have no competing interests.

\section{Author details}

${ }^{1}$ Department of Environmental Sciences, Informatics and Statistics, Ca' Foscari University of Venice, Via Torino 155, 30172, Mestre, Venezia, Italy.

${ }^{2}$ Autonomous University of Barcelona, Institute of Environmental Science and Technology, 08193, Bellaterra, Cerdanyola del Vallès, Spain. ${ }^{3}$ University of

Gastronomic Sciences, Piazza Vittorio Emanuele II 9, 12042 Pollenzo/Bra, Italy.

${ }^{4}$ Medical Analysis Department, TISHK International University, Qazi

Muhammad, Erbil, Kurdistan 44001, Iraq.

Received: 21 April 2020 Accepted: 12 June 2020

Published online: 10 July 2020

\section{References}

1. Hopping K, Yangzong C, Klein J. Local knowledge production, transmission, and the importance of village leaders in a network of Tibetan pastoralists coping with environmental change. Ecol Soc. 2016;21(1).

2. Mamun AA. Understanding the value of local ecological knowledge and practices for habitat restoration in human-altered floodplain systems: a case from Bangladesh. Environ Manag. 2010;45(5):922-38.

3. Naess LO. The role of local knowledge in adaptation to climate change. Wiley Interdiscip Rev Clim Chang. 2013;4(2):99-106.

4. Folke C, Jansson Å, Rockström J, Olsson P, Carpenter SR, Chapin FS, Crépin AS, Daily G, Danell K, Ebbesson J, Elmqvist T. Reconnecting to the biosphere. Ambio. 2011;40(7):719.

5. Cruz García GS. The mother-child nexus. Knowledge and valuation of wild food plants in Wayanad, Western Ghats, India. J Ethnobiol Ethnomed. 2006; 2(1):39.

6. Pieroni A, Sõukand R. Are borders more important than geographical distance? The wild food ethnobotany of the Boykos and its overlap with that of the Bukovinian Hutsuls in Western Ukraine. J Ethnobiol. 2017;37(2): 326-46.

7. Mamedov N, Gardner Z, Craker LE. Medicinal plants used in Russia and Central Asia for the treatment of selected skin conditions. J Herbs Spices Med Plants. 2005;11(1-2):191-222. 
8. Olsson P, Folke C. Local ecological knowledge and institutional dynamics for ecosystem management: a study of Lake Racken watershed, Sweden. Ecosystems. 2001;4(2):85-104.

9. Berkes F, Colding J, Folke C. Rediscovery of traditional ecological knowledge as adaptive management. Ecol Appl. 2000;10(5):1251-62.

10. van den Boog T, van Andel T, Bulkan J. Indigenous children's knowledge about non-timber forest products in Suriname. Econ Bot. 2017;71(4):361-73.

11. Reyes-García V, Vadez V, Huanca T, Leonard W, Wilkie D. Knowledge and consumption of wild plants: a comparative study in two Tsimane'villages in the Bolivian Amazon. Ethnobot Res Appl. 2005;3:201-7.

12. McMillen $\mathrm{H}$. Ethnobotanical knowledge transmission and evolution: the case of medicinal markets in Tanga, Tanzania. Econ Bot. 2012;66(2):121-31.

13. Zarger R, Stepp J. Persistence of botanical knowledge among Tzeltal Maya children. Curr Anthropol. 2004;45(3):413-8.

14. Saghin D, Matei E, Cojocaru I, Manea G, Cocoş O. The Hutsuls in South Bukovina: from rural tradition to sustainable development. East Eur Countryside. 2017;23(1):119-45.

15. Figlus T. Past and present of Hutsulshchyna as the Carpathian borderland region. Remarks on changes of spatial structures, ethno-cultural specificity and heritage. Państwowy Instytut Naukowy-Instytut Śląski w Opolu, Wydawnictwo Instytut Śląski, Katedra Geografii Politycznej i Studiów Regionalnych Uniwersytetu Łódzkiego. 2009;9:143-59.

16. Sõukand $\mathrm{R}$, Pieroni $A$. The importance of a border: medical, veterinary, and wild food ethnobotany of the Hutsuls living on the Romanian and Ukrainian sides of Bukovina. J Ethnopharmacol. 2016;185:17-40.

17. Ozturk M, Altay V, Altundağ E, Ibadullayeva SJ, Aslanipour B, Gönenç TM Herbals in lğdır (Turkey), Nakhchivan (Azerbaijan), and Tabriz (Iran). Plant Human Health. 2018;1:197-266

18. Akgul A, Akgul A, Senol SG, Yildirim H, Secmen O, Dogan Y. An ethnobotanical study in Midyat (Turkey), a city on the silk road where cultures meet. J Ethnobiol Ethnomed. 2018;14:1-12.

19. Fisher G, Röger M. Bukovina: a borderland region in (trans-) national historiographies after 1945 and 1989-1991. East Eur Pol Soc. 2019;33(1):176-95.

20. Kot T. Region of Bukovina. The heritage of the Europe in miniature. Region and regionalism. Regions in the process of European integration. Multicult Regions Cities Context Eur Integration. 2007;(8).

21. Kołodziejska-Degórska I. Mental herbals-a context-sensitive way of looking at local ethnobotanical knowledge: examples from Bukovina (Romania). Trames. 2012;16(3):287-301.

22. The Plant List. 2013 Available at: http://www.theplantlist.org/. Accessed 23. 03.2020 .

23. Tutin T, Heywood V, Burges N, Valentine D, Walters S, Webb D. Flora Europaea: Cambridge University Press; 1964.

24. Stevens PF. Angiosperm phylogeny website, version 14. 2017. Available at: http://www.mobot.org/MOBOT/research/APweb. Accessed 23.03.2020.

25. Slovnyk (2010) Official Ukrainian transliteration. Available at https://slovnyk ua/translit.php.

26. World Health Organization. ICD-11 for Mortality and Morbidity Statistics 2018 version. Available at https://icd.who.int/browse11//-m/en Accessed 23. 03.2020 .

27. González-Tejero MR, Casares-Porcel M, Sánchez-Rojas CP, Ramiro-Gutiérrez JM, Molero-Mesa J, Pieroni A, Giusti ME, Censorii E, De Pasquale C, Della A, ParaskevaHadijchambi D. Medicinal plants in the Mediterranean area: synthesis of the results of the project Rubia. J Ethnopharmacol. 2008;116(2):341-57.

28. Sõukand R, Quave CL, Pieroni A, Pardo-de-Santayana M, Tardío J, Kalle R, et al. Plants used for making recreational tea in Europe: a review based on specific research sites. J Ethnobiol Ethnomed. 2013;9:58.

29. Treben M. Sănătate din Farmacia Domnului: Hunga-print; 1980.

30. Mohan G. Atlasul plantelor medicinale din România. Bucharest: Corint; 2001.

31. Davis CM, Charemza W. Models of disequilibrium and shortage in centrally planned economies: Springer Science \& Business Media; 2002.

32. Hovsepyan R, Stepanyan-Gandilyan N, Stollberg C. Phytomedicinal knowledge and "official" sources in Tatev (Armenia). Ethnobiol Lett. 2019; 10(1):23-34.

33. Coyne EV. Coming down from the mountain: dialect contact and convergence in contemporary Hutsulshchyna (Doctoral dissertation, UC Berkeley); 2014.

34. Vronskaya T. Responsive actions: a new stage of extra-judicial repression of rebel relatives (1948-1949); 2009.

35. Handa SS, Rakesh DD, Vasisht K. Compendium of medicinal and aromatic plants Asia. ICS UNIDO Asia. 2006;2:305.
36. Krasheninnikova Y. Informal healthcare in contemporary Russia: sociographic essays on the post-soviet infrastructure for alternative healing practices: Columbia University Press; 2017.

37. Kołodziejska-Degórska I. Patients' webs of relations in the medical landscapes of Central Ukraine. Anthropol Med. 2016;23(2):155-71.

38. Kibych A. Hutsulshchyna and public progress. Bulletin of geography. Socioeconomic Ser. 2010;14(14):37-50.

39. Kliucharev G, Morgan WJ. Non-governmental organizations, non-formal education and civil society in contemporary Russia. In: China's opening society: the non-state sector and governance; 2008. p. 54-70.

40. Bodnar $V$. The soviet regime repression against orthodox church in Bukovyna region in 1940-1960s. Mod Sci Mod Věda. 2015;3:65-72.

41. Chervinska I, Nykorak J, Chervinskyi A. The traditions and beliefs of highlanders as a means of self-identity and ethnos preserving. J Vasyl Stefanyk Precarpathian Natl Univ. 2017:4(1):79-86.

42. Nowak A, Tokarczyk N. Transformations of traditional land use and settlement patterns of Kosarysche ridge (Chornohora, Western Ukraine). Bulletin of geography. Socioeconomic Ser. 2014;24(24):191-201.

43. Connor GS. Rural systematization: a radical campaign of rural planning under Ceausescu regime in Romania. Int J Bus Soc Sci. 2017;8(2):15-20.

44. World Bank Physicians (per 1,000 people). 2020. Available at: https://data. worldbank.org/indicator/SH.MED.PHYS.ZS? contextual=region\&end=2014 \&locations=UA\&start=1980\&view=chart Accessed 23.03.2020.

45. Kokotkiewicz A, Jaremicz Z, Luczkiewicz M. Aronia plants: a review of traditional use, biological activities, and perspectives for modern medicine. $J$ Med Food. 2010;13(2):255-69.

46. Panossian A, Wagner H. From traditional to evidence-based use of Hippophae rhamnoides L.: chemical composition, experimental, and clinical pharmacology of sea buckthorn berries and leaves extracts, Evidence and rational based research on Chinese drugs. Vienna: Springer; 2013. p. 181236.

47. Fedorak V. Osoblyvosti ta perspectyvy rozvytku etnoturyzmu na hucul'ščyni. In: Monolatij I, editor. Ukraijna, halyčyna, hucul'ščyna: Istorija, polityka, kultura; 2009. p. 187-9.

\section{Publisher's Note}

Springer Nature remains neutral with regard to jurisdictional claims in published maps and institutional affiliations.

Ready to submit your research? Choose BMC and benefit from

- fast, convenient online submission

- thorough peer review by experienced researchers in your field

- rapid publication on acceptance

- support for research data, including large and complex data types

- gold Open Access which fosters wider collaboration and increased citations

- maximum visibility for your research: over $100 \mathrm{M}$ website views per year

At $\mathrm{BMC}$, research is always in progress.

Learn more biomedcentral.com/submissions 